INSTITUTO DE PESQUISAS ENERGÉTICAS E NUCLEARES Autarquia Associada à Universidade de São Paulo

\title{
APLICAÇÃO DO MÉTODO DE MONTE CARLO NO ESTUDO DA PADRONIZAÇÃO DE RADIONUCLÍDEOS COM ESQUEMA DE DESINTEGRAÇÃO COMPLEXOS EM SISTEMA DE COINCIDÊNCIAS $4 \pi \beta-\gamma$
}

MAURO NORIAKI TAKEDA

Tese apresentada como parte dos requisitos para obtenção do grau de Doutor em Ciências na Área de Tecnologia Nuclear - Aplicações.

Orientador:

Dr. Mauro da Silva Dias 
À minha esposa Fátima

Aos meus filhos, Lucas e Artur

Aos meus pais, Seiki e Sakae 


\section{Agradecimentos:}

Ao Dr. Mauro da Silva Dias, orientador deste trabalho, pela oportunidade, apoio, paciência e dedicação, durante o desenvolvimento desta tese;

À Dra. Marina Fallone Koskinas, pelas discussões, sugestões e apoio durante todo o desenvolvimento do trabalho;

Aos colegas Aparecido Edílson Morcelli, Carla Costa Cardoso Zoppe e Karl Friehe pelo apoio e colaboração;

Aos colegas Franco Brancaccio, Cláudio Domienikan, Fábio de Toledo, Hélio Piuvezam Filho, Carlos Augusto Pires e Eliezer Antonio da Silva pela amizade, apoio, estímulo e colaboração;

Às colegas Cláudia Regina Ponte Ponge Ferreira e Denise Simões Moreira pela amizade, apoio, incentivo e colaboração;

Ao Dr. Pablo Arenillas do Laboratório de Metrologia de Radioisótopos, Comisión Nacional de Energía Atómica, Argentina, pelas várias sugestões.

Ao Instituto de Pesquisas Energéticas e Nucleares, IPEN-CNEN/SP, na pessoa do Superintendente Dr. Cláudio Rodrigues pela possibilidade oferecida para o desenvolvimento deste trabalho;

A minha esposa Fátima, pelo apoio, incentivo, compreensão e paciência durante a realização deste trabalho;

Aos meus filhos Lucas e Artur, que foram a fonte de energia para a realização deste trabalho;

Aos meus pais que sempre apoiaram e incentivaram os meus estudos;

Ao pessoal da Comissão de Pós-Graduação do IPEN-CNEN/SP pelo apoio oferecido;

A todos que direta ou indiretamente colaboraram na execução e realização deste trabalho. 


\title{
APLICAÇÃO DO MÉTODO DE MONTE CARLO NO ESTUDO DA PADRONIZAÇÃO DE RADIONUCLÍDEOS COM ESQUEMA DE DESINTEGRAÇÃO COMPLEXOS EM SISTEMA DE COINCIDÊNCIAS $4 \pi \beta-\gamma$
}

\author{
MAURO NORIAKI TAKEDA
}

\section{RESUMO}

O presente trabalho descreve uma nova metodologia desenvolvida para modelar o comportamento da atividade em sistema de coincidência $4 \pi(\mathrm{PC}) \beta-\gamma$. As eficiências para elétrons no detector proporcional (PC) e para radiação gama no detector de $\mathrm{NaI}(\mathrm{Tl})$ foram calculadas utilizando o programa de Monte Carlo MCNP4C. Outro código de Monte Carlo foi desenvolvido para seguir o caminho no esquema de desintegração desde o estado inicial do radionuclídeo precursor, até o estado fundamental do núcleo filho. Cada etapa do esquema de desintegração é selecionada por meio de números aleatórios levando em conta as probabilidades de cada ramo $\beta^{-}$ou captura eletrônica, as probabilidades de transição e os coeficientes de conversão interna. Uma vez que o estado final tenha sido atingido é verificado se houve detecção de eventos beta, captura eletrônica ou transições gama, e os eventos para os três espectros, beta, gama e coincidência são contabilizados. A variação da eficiência beta pode ser feita através da simulação de corte na energia detectada ou pela utilização de absorvedores (Collodion). Foram escolhidos para a simulação os radionuclídeos ${ }^{134} \mathrm{Cs},{ }^{72} \mathrm{Ga}$ que se desintegram por transição $\beta-{ }^{133} \mathrm{Ba}$ que se desintegra por captura eletrônica e ${ }^{35} \mathrm{~S}$ que é um emissor beta puro. Para este último foi simulando o Método do Traçador. As curvas de extrapolação obtidas por Monte Carlo foram ajustadas pelo Método dos Mínimos Quadrados com os pontos experimentais e comparados com os resultados obtidos pelo Método da Extrapolação Linear. 


\title{
APPLICATION OF MONTE CARLO METHOD IN STUDY OF THE PADRONIZATION FOR RADIONUCLIDES WITH COMPLEX DISINTEGRATION SCHEME IN $4 \pi \beta-\gamma$ COINCIDENCE SYSTEM
}

\author{
MAURO NORIAKI TAKEDA
}

\begin{abstract}
The present work describe a new methodology for modelling the behaviour of the activity in a $4 \pi(\mathrm{PC}) \beta-\gamma$ coincidence system. The detection efficiency for electrons in the proportional counter and gamma radiation in the $\mathrm{NaI}(\mathrm{Tl})$ detector was calculated using the Monte Carlo program MCNP4C. Another Monte Carlo code was developed which follows the path in the disintegration scheme from the initial state of the precursor radionuclide, until the ground state of the daughter nucleus. Every step of the disintegration scheme is sorted by random numbers taking into account the probabilities of all $\beta^{-}$branches, electronic capture branches, transitions probabilities and internal conversion coefficients. Once the final state was reached beta, electronic capture events and gamma transitions are accounted for the three spectra: beta, gamma and coincidence variation in the beta efficiency was performed simulating energy cut off or use of absorbers (Collodion). The selected radionuclides for simulation were: ${ }^{134} \mathrm{Cs}$, ${ }^{72} \mathrm{Ga}$ which disintegrate by $\beta^{-}$transition, ${ }^{133} \mathrm{Ba}$ which disintegrates by electronic capture and ${ }^{35} \mathrm{~S}$ which is a beta pure emitter. For the latter, the Efficiency Tracing technique was simulated. The extrapolation curves obtained by Monte Carlo were filled by the Least Square Method with the experimental points and the results were compared to the Linear Extrapolation method.
\end{abstract}




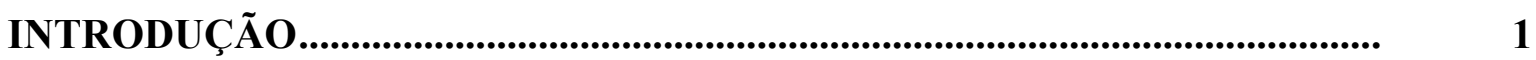

1. FUNDAMENTOS TEÓRICOS .................................................................. 6

1.1. Processos de Desintegração Radioativa.................................................. 6

1.2. Lei da Desintegração Radioativa ............................................................. 8

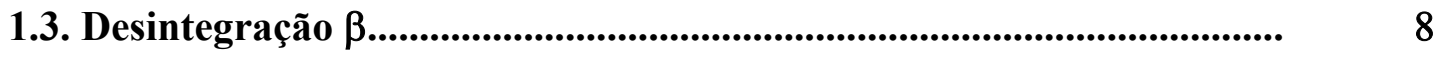

1.4. Captura Eletrônica (CE)........................................................................ 12

1.5. Desexcitação de Estados do Núcleo Filho.............................................. 12

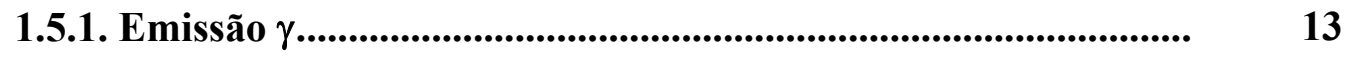

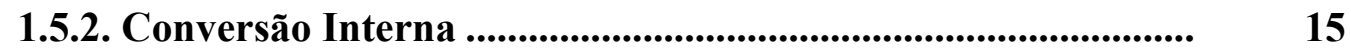

1.6. Rearranjo Eletrônico ........................................................................... 17

1.7. Interação da Radiação com a Matéria ................................................ $\quad 20$

1.7.1. Efeito Fotoelétrico ............................................................... 22

1.7.2. Efeito Compton ................................................................................ 24

1.7.3. Produção-de-Pares ................................................................... 26

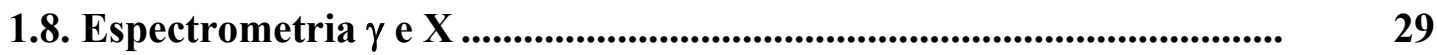

2. METODOLOGIA............................................................................................. 33

2.1. Medida Absoluta da Atividade pelo Método de Coincidência ............ 33

2.1.1. Princípio do Método ............................................................... 33

2.1.2. Técnica da Extrapolação da Eficiência ....................................... 35

2.1.3. Técnica do Traçador................................................................ 39

2.2. Arranjo Experimental do Sistema de Coincidência $4 \pi(\mathrm{PC}) \beta-\gamma \quad \ldots . . . . . \quad 41$

2.2.1. Detector Proporcional com Geometria $4 \pi$.............................. 42

2.2.2. Cristal Cintilador de $\mathrm{NaI}(\mathrm{Tl})$................................................ 43

2.2.3. Sistema Eletrônico Associado ....................................................... 44

2.2.4 Preparação de Fontes Radioativas ............................................. 46

2.3. Simulação Teórica do Sistema de Coincidência $4 \pi(\mathrm{PC}) \beta-\gamma \ldots . . . . . . . . . . . . . \quad 47$

2.4. Padronização de Radionuclídeos por Meio de Sistemas de

Coincidência ................................................................................................... 48

2.4.1 Padronização do ${ }^{134}$ Cs...................................................................... 48

2.4.2. Padronização do ${ }^{72} \mathrm{Ga}$............................................................. 50 
2.4.3. Padronização do ${ }^{133}$ Ba ............................................................. 54

2.4.4. Padronização do ${ }^{35} \mathrm{~S}$............................................................... 57

2.5. O Método de Monte Carlo .................................................................. 58

2.5.1. Introdução .......................................................................... 58

2.5.2. Problemas que Podem ser Resolvidos pelo Método de Monte Carlo ................................................................................ 59

2.5.3. Números Aleatórios e Pseudo-Aleatórios.................................... 60

2.6. Aplicação do Método de Monte Carlo ao Presente Trabalho .............. 62

2.6.1. Período e Uniformidade na Geração de Números Aleatórios do Gerador de Números Aleatórios do FORTRAN ................. 63

2.6.2. Elaboração dos Dados de Entrada Utilizados no Programa ESQUEMA …................................................................... $\quad 65$

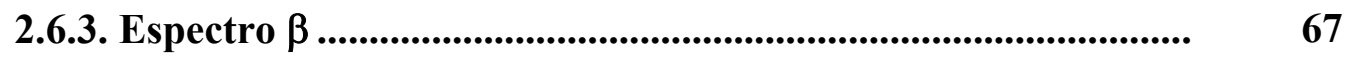

2.6.4. Resolução em Energia................................................................... 68

2.6.5. O Programa ESQUEMA ..................................................... 69

2.6.5.1. Desintegração por Captura Eletrônica........................... 71

2.6.5.2. Desintegração por $\beta^{-}$......................................... 72

2.6.5.3. Transição $\gamma$ ou Elétron de Conversão Interna .............. 72

2.6.5.4. Espectros $\beta$, $\gamma$ e Coincidência......................................... 73

2.6.6. Determinação da Atividade pelo Método de Monte Carlo...... 74

2.6.7. Simulações Iniciais ............................................................... $\quad 75$

3. RESULTADOS E DISCUSSÃO_.................................................................... $\quad 80$

3.1. Padronização do ${ }^{134}$ Cs ........................................................................... 80

3.2. Padronização do ${ }^{72} \mathrm{Ga}$........................................................................... 82

3.3. Padronização do ${ }^{133}$ Ba......................................................................... 84

3.4. Padronização do ${ }^{35}$ S............................................................................... 87

4. CONCLUSÃO ................................................................................................ 89

5. PERSPECTIVAS FUTURAS ..................................................................... 91

APÊNDICE A - Código para Verificar o Período do Gerador de Números Aleatórios do Fortran.................................................................... 93

APÊNDICE B - Código para Verificar a Uniformidade na Geração de

Números Aleatórios do Fortran ....................................................... 96

APÊNDICE C - Arquivo EFIBETA.DAT ........................................................ 99 
APÊNDICE D - Arquivo EFIGAMA.DAT ............................................................ 100

APÊNDICE E - Arquivo EFIGAMAT.DAT ...................................................... 101

APÊNDICE F - Arquivo CONSTANT.DAT ....................................................... 102

APÊNDICE G - Arquivo ESQUEMA.DAT ........................................................... 103

APÊNDICE H - Arquivo NPICOS.DAT ............................................................. 105

APÊNDICE I - Arquivo FERMI.DAT............................................................... 106

APÊNDICE J - Código do Programa ESQUEMA ................................................. 107

APÊNDICE K - Arquivo RANGE.DAT .............................................................. 109

REFERÊNCIAS BIBLIOGRÁFICAS............................................................... 110 


\section{INTRODUÇÃO}

Em Metrologia Nuclear, o desenvolvimento e a utilização do método de medida absoluta de atividade de radionuclídeos por coincidência $4 \pi \beta-\gamma$ iniciou-se nas décadas de 60 e 70, quando o progresso na fabricação de detectores e o desenvolvimento na área de eletrônica permitiram o uso de sistemas de medidas mais confiáveis, sendo atualmente considerado um padrão primário, em razão de seu alto grau de exatidão e por depender apenas de grandezas observáveis para a obtenção de seus resultados ${ }^{[1-7]}$. Usualmente, um sistema deste tipo é constituído por um detector proporcional em geometria $4 \pi$, a gás fluente ou pressurizado, para a detecção de partículas alfa, beta, elétrons ou fóton-X, acoplado a um espectrômetro gama, que utiliza detectores de cintilação ou cristais semicondutores ${ }^{[8-10]}$. Outras configurações envolvem cintiladores líquidos $^{[11-15]}$ ou plásticos ${ }^{[16,17]}$, para serem utilizados como detectores $4 \pi$.

O Laboratório de Metrologia Nuclear (LMN) do IPEN-CNEN/SP, desde a sua fundação em 1964, tem desenvolvido diversos sistemas e metodologias de medida de atividade de radionuclídeos ${ }^{[18-29]}$. A confiabilidade dessas padronizações tem sido verificada por meio de várias comparações internacionais patrocinadas pelo BIPM (Bureau International dès Poids et Mèsures), França ${ }^{[30-36]}$, cujos resultados comprovam o excelente grau de confiabilidade atingido pelos sistemas de medidas desenvolvidos no LMN do IPEN.

Atualmente, o LMN possui dois sistemas de coincidência $4 \pi \beta-\gamma$ constituídos por detectores proporcionais a gás fluente e pressurizado, respectivamente ${ }^{[7,24]}$. Estes sistemas são considerados primários e servem como referência para os diversos sistemas de calibração secundários do LMN, constituídos por: câmaras de ionização, detectores de barreira de superfície, espectrômetros de HPGe ou Ge(Li) e cintiladores de $\mathrm{NaI}(\mathrm{Tl})$.

Uma das dificuldades da utilização do método de coincidências $4 \pi \beta-\gamma$ é o planejamento detalhado das condições experimentais que possibilitem a otimização das 
medidas, de modo a minimizar a incerteza no valor da atividade. Usualmente, este valor é obtido pelo Método da Extrapolação ${ }^{[3]}$, medindo-se o comportamento da atividade da fonte radioativa, em função de um parâmetro associado à eficiência do detector $4 \pi \beta$. O valor extrapolado para eficiência unitária corresponde à atividade procurada.

Para esquemas de desintegração simples, este planejamento é relativamente fácil e os resultados podem ser obtidos de modo rápido e confiável. Entretanto, para esquemas complexos, torna-se difícil definir a priori as condições ideais de medida. Neste caso, diversos efeitos espectrais podem ocorrer, tais como: degradação da energia da radiação, sobreposição de eventos provenientes de radiações de natureza diferentes, tanto no detector $4 \pi \beta$ como no detector gama. Desta forma, o planejamento deve levar em conta tanto as características do esquema de desintegração, como a contribuição de cada uma das radiações emitidas no espectro final dos detectores.

Na literatura, a abordagem é feita, em geral, de forma analítica e usualmente considera apenas os parâmetros do esquema de desintegração, sem considerar em detalhe as eficiências dos detectores. Em alguns casos, uma parte da expressão analítica do Método de Coincidências, onde está incluída a eficiência do detector gama, pode ser estimada teoricamente ${ }^{[37-39]}$ para o caso de um radionuclídeo específico.

Na padronização do ${ }^{169} \mathrm{Yb}$ da referência 37 o autor considerando as condições ideais de medida simulou a desintegração matematicamente. Para simplificar os cálculos, utilizou os seguintes parâmetros: a "intensidade dos níveis", $\mathrm{I}_{\mathrm{i}}$, (i.e., a soma das intensidades absolutas das transições para esse nível) e a "eficiência dos níveis", $\varepsilon_{i}$, (i.e. a parte da desintegração desse nível computado no canal considerado, incluindo o canal coincidência). Os parâmetros de cada nível são calculados usando os parâmetros das transições para esse nível e dos níveis abaixo dele.

O autor da referência 38, na padronização do ${ }^{169} \mathrm{Yb}$, utiliza somente parte da fórmula de coincidência, na qual é levado em consideração as eficiências do detector $\beta$ para a radiação $\beta$ do r-ésimo ramo $\beta$ e a eficiência do detector $\beta$ para a transição gama (elétrons de conversão ou fótons) associados ao r-ésimo ramo $\beta$. 
No presente trabalho, a abordagem é abrangente, possibilitando a sua utilização para qualquer radionuclídeo de interesse, cujo esquema de desintegração seja conhecido com a exatidão requerida. Nesta abordagem, as informações contidas no esquema de desintegração são utilizadas para determinar a contribuição de todas as radiações emitidas pelo radionuclídeo nos espectros medidos em cada um dos detectores. A simulação desenvolvida permite uma previsão do espectro de coincidências, possibilitando a escolha adequada das energias gama para as quais a atividade possa ser obtida com máxima exatidão.

O propósito do presente trabalho foi desenvolver uma metodologia, aplicando a técnica de Monte Carlo, para simular todo o processo de detecção em um sistema de coincidência $4 \pi(\beta, X)-\gamma$, possibilitando o conhecimento prévio do comportamento da atividade aparente da fonte radioativa como função da eficiência do detector $4 \pi \beta$. Os resultados foram comparados a curvas experimentais obtidas para alguns radionuclídeos padronizados no LMN do IPEN.

A relevância do tema justifica-se pela necessidade de um planejamento adequado das medidas, particularmente para radionuclídeos com esquema de desintegração complexos. O comportamento da expressão analítica geral do Método de Coincidências $4 \pi(\beta, X)-\gamma$ é determinado por meio dos espectros simulados para as vias $\beta, \gamma$ e de coincidência.

Os aspectos de originalidade estão associados ao desenvolvimento da metodologia teórica utilizando o método de Monte Carlo para a simulação dessa expressão analítica, considerando a história da radiação desde a sua emissão até o registro do pulso no detector. Esta simulação possibilita prever detalhadamente o comportamento da curva de extrapolação, principalmente na região de eficiências do detector $4 \pi(\beta, X)$ próximas de $100 \%$, inacessível experimentalmente em razão de efeitos de auto-absorção dos elétrons de baixa energia no substrato da fonte radioativa.

O método proposto torna-se complexo, principalmente na simulação de radionuclídeos que se desintegram por Captura de Elétrons, ou emissão de radiação beta de baixa energia, em presença de gamas de alta energia. Além disso, a forma do espectro de 
energia das radiações beta é obtida a partir da teoria geral aplicada a desintegração $\operatorname{beta}^{[40,41]}$.

Os radionúclideos estudados na padronização de radionuclídeos por meio de sistemas de coincidência $4 \pi \beta-\gamma$ aplicando o Método de Monte Carlo apresentam desintegração por $\beta-\gamma, \mathrm{CE}-\gamma$, e emissor beta puro, este aplicando a técnica do traçador. Foi escolhido o ${ }^{134} \mathrm{Cs}$ e ${ }^{72} \mathrm{Ga}$, que desintegram por $\beta^{-} ;{ }^{133} \mathrm{Ba}$, que desintegra por captura eletrônica e o ${ }^{35} \mathrm{~S}$ que é um emissor beta puro.

$\mathrm{O}{ }^{134} \mathrm{Cs}$ apresenta um esquema de desintegração complexo e desintegra-se por $\beta^{-}$seguida de várias transições gama ou elétrons de conversão interna. $\mathrm{O}{ }^{72} \mathrm{Ga}$ também apresenta um esquema de desintegração complexo e desintegra-se por $\beta^{-}$seguida de várias transições gama ou elétrons de conversão interna, e é de interesse em razão da presença de gamas de $2200 \mathrm{keV}$ e $2500 \mathrm{keV}$, preenchendo um intervalo de energias onde há poucos pontos de calibração e pode ser utilizado como um padrão auxiliar. $\mathrm{O}{ }^{133} \mathrm{Ba}$ apresenta um esquema de desintegração complexo e se desintegra por captura eletrônica seguida de várias transições gama ou elétrons de conversão interna, e foi alvo de uma comparação internacional ${ }^{[35]}$. $\mathrm{O}{ }^{35} \mathrm{~S}$ é um emissor beta puro e foi utilizado o ${ }^{60} \mathrm{Co}$ como traçador, e permitiu a simulação por Monte Carlo da Técnica do Traçador na padronização de radionuclídeos.

A escolha desses radionuclídeos permitiu fazer um estudo dos resultados obtidos aplicando-se o Método de Monte Carlo comparado com os resultados experimentais para esses radionuclídeos, e verificar a validade do código de Monte Carlo desenvolvido no presente trabalho para simular todo o processo de detecção do sistema de coincidência $4 \pi \beta-\gamma$ para os casos estudados.

No Capítulo 1 são apresentados os fundamentos teóricos envolvendo os processos de desintegração radioativa ressaltando a desintegração beta e Captura eletrônica, a desexcitação de um núcleo atômico, o rearranjo eletrônico e a interação da radiação com a matéria. 
No Capítulo 2 é tratada a metodologia empregada e aborda a medida absoluta da atividade pelo método de coincidência, o arranjo experimental do sistema de coincidência $4 \pi(\mathrm{PC}) \beta-\gamma$, a preparação das fontes radioativas, padronização de radionuclídeos por meio de sistemas de coincidência, o método de Monte Carlo e a simulação por Monte Carlo aplicando a teoria de Fermi para o espectro beta, desenvolvimento do programa ESQUEMA para a determinação da atividade pelo método de Monte Carlo.

No Capítulo 3 são apresentados os resultados obtidos utilizando o método de Monte Carlo na padronização de radionuclídeos por meio do sistema de coincidência $4 \pi(\mathrm{PC}) \beta-\gamma$ e feito uma discussão desses resultados.

No Capítulo 4 são apresentas as conclusões deste trabalho.

No Capítulo 5 são apresentadas as perspectivas futuras que podem ser implementadas no presente trabalho visando um aperfeiçoamento e refinamento do modelo utilizado. 


\section{FUNDAMENTOS TEÓRICOS}

Neste capítulo são abordados os fundamentos teóricos sobre os processos de desintegração radioativa e os vários tipos de radiações com diferentes características provenientes desses processos, além de considerações sobre a interação da radiação com a matéria. É apresentado o método de coincidência e o método de Monte Carlo, essenciais para o desenvolvimento deste trabalho.

\subsection{Processos de Desintegração Radioativa}

Um nuclídeo radioativo tende a seu estado fundamental pela desintegração radioativa que ocorre por diferentes processos atômicos ou nucleares que dão origem a radiações que são classificadas em quatro tipos gerais a seguir ${ }^{[42]}$ :

$$
\begin{aligned}
& \text { radiação de partículas carregadas }\left\{\begin{array}{l}
\text { elétrons } \\
\text { partículas carregadas pesadas }
\end{array}\right. \\
& \text { radiação sem c arg a }\left\{\begin{array}{l}
\text { radiação eletromagnética } \\
\text { nêutrons }
\end{array}\right.
\end{aligned}
$$

Dentre essas radiações, as partículas carregadas que são de interesse no presente trabalho incluem: partícula beta (positiva ou negativa), captura eletrônica, elétrons de conversão interna, elétrons Auger. As radiações eletromagnéticas incluem: raio X emitido no rearranjo das camadas eletrônicas do átomo, e raios gama que originam de transições dentro do próprio núcleo.

O principal enfoque dessas radiações é com relação às fontes radioativas de baixa atividade $(\mathrm{kBq})$ que são de interesse para a calibração de detectores de radiação ou caracterização das propriedades de desintegração dos radionuclídeos.

As radiações de interesse diferem pela dificuldade ou capacidade de penetração no material. Radiações de baixo poder de penetração, como partículas alfa ou raio-X e 
elétrons de baixa energia, conseguem atravessar somente pequenas espessuras de material. Fontes radioativas espessas podem portanto, depositar uma fração considerável da energia dessas radiações no substrato da própria fonte. Este efeito, denominado auto-absorção, altera a intensidade e o espectro de energia das radiações que emergem de sua superfície. Partículas beta de alta energia são geralmente mais penetrantes, e fontes que apresentem alguns centésimos de milímetros de espessura, em geral podem ser aceitas. Radiações de alto poder de penetração, tais como raios gama, de energias intermediárias ou altas são muito menos afetadas pela auto-absorção e as fontes podem ter dimensões de décimos de milímetro, sem afetar seriamente as características do feixe de radiação ${ }^{[42]}$.

As diferentes propriedades de desintegração dos radionuclídeos e os parâmetros característicos de sua desintegração são normalmente descritos pelos esquemas de desintegração, que são diagramas que apresentam a energia de desintegração, as energias dos níveis excitados e as transições entre níveis excitados, conforme exemplo da figura 1.1 .

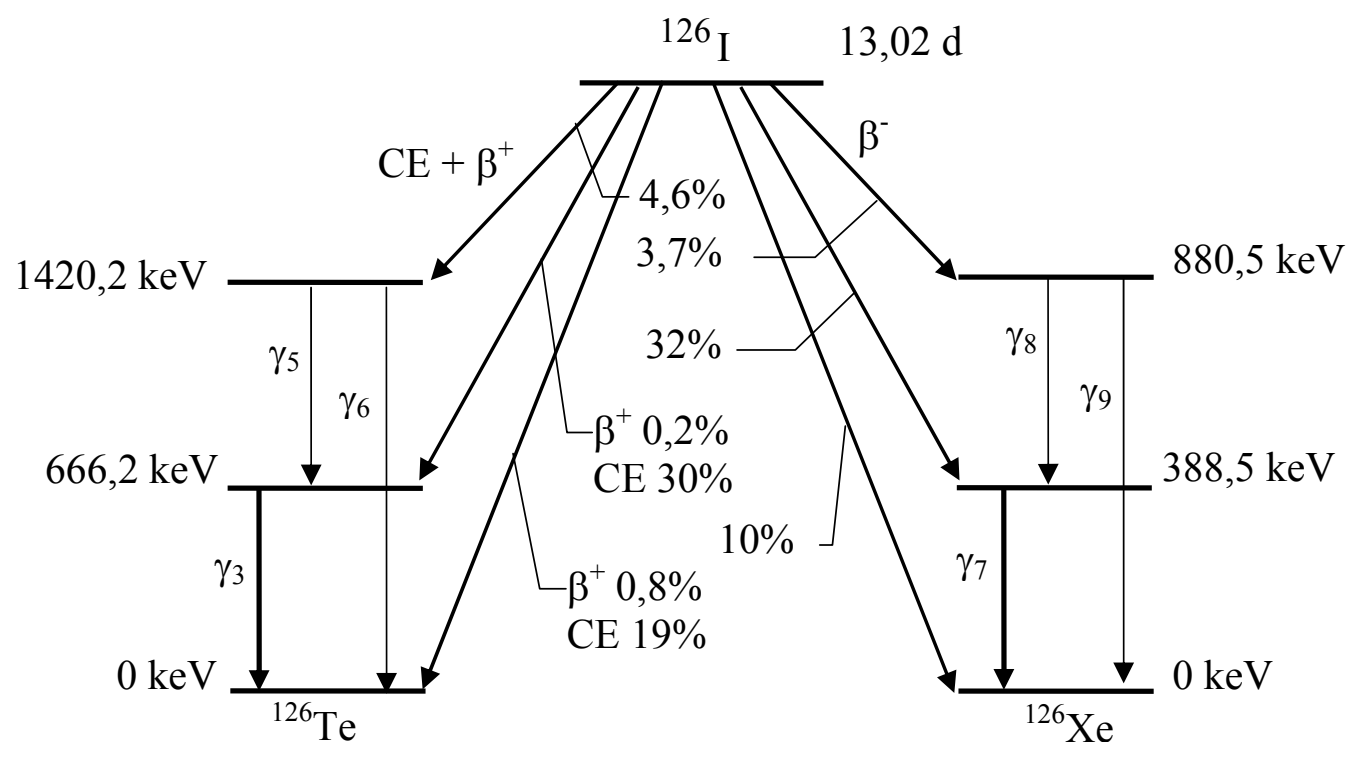

Figura 1.1 - Esquema de desintegração típico. 


\subsection{Lei da Desintegração Radioativa}

A atividade de uma fonte radioativa é definida como a sua taxa de desintegração e é obtida da lei fundamental da desintegração radioativa, para um radionuclídeo isolado:

$$
\frac{d N}{d t}=-\lambda N
$$

na qual $\mathrm{N}$ é o número de núcleos radioativos e $\lambda$ é definido como constante de desintegração. De acordo com a equação 1.1, o decréscimo no número de átomos do elemento radioativo por unidade de tempo é proporcional ao número de átomos ainda não desintegrado.

A unidade de atividade no Sistema Internacional é o becquerel $(\mathrm{Bq})$, e é definido como uma desintegração por segundo, assim:

$$
1 \mathrm{~Bq}=1 \mathrm{~s}^{-1}
$$

A quantidade atividade é definida pelo $\mathrm{NCRP}^{[43]}$ da seguinte forma: "A quantidade da atividade de um nuclídeo em um estado de energia específico em um dado instante de tempo é o valor esperado do número de transições nucleares espontâneas por unidade de tempo daquele estado de energia".

A atividade específica de uma fonte radioativa é definida como a atividade por unidade de massa da amostra radioativa, dado $\mathrm{em} \mathrm{Bq} \cdot \mathrm{g}^{-1}$.

\subsection{Desintegração $\beta$}

A desintegração $\beta$ pode manifestar-se de duas formas, através da emissão de um elétron negativo ou um pósitron do núcleo. As reações para esses processos são apresentadas a seguir de forma simplificada, visto que o detalhamento de como ocorrem esses processos foge ao escopo do presente trabalho. 
$\mathrm{O}$ valor de $\mathrm{Q}$ indicado representa a energia liberada no processo. $\mathrm{Na}$ desintegração por emissão de um elétron negativo, o número atômico é incrementado de uma unidade, como mostra a equação $1.2^{[44]}$.

$$
{ }_{Z}^{A} X \rightarrow{ }_{Z+1}^{A} Y+\beta^{-}+\bar{v}+Q
$$

$\bar{v}$ representa o antineutrino.

Na emissão de um pósitron, resulta no decréscimo do número atômico de uma unidade, como na reação indicada na equação $1.3^{[44]}$.

$$
{ }_{Z}^{A} X \rightarrow{ }_{Z-1}^{A} Y+\beta^{+}+v+Q
$$

em que $v$ representa o neutrino.

- Desintegração $\beta^{-}$: Neste processo um nêutron é convertido em um próton, e uma partícula $\beta^{-}$e um antineutrino (que tem massa de repouso aproximadamente nula e probabilidade extremamente pequena de interação com a matéria) são emitidos do núcleo como resultado do processo ${ }^{[44]}$ :

$$
n \rightarrow p+e^{-}+\bar{v}
$$

Essa desintegração aumenta a carga nuclear em uma unidade.

Para alguns nuclídeos, a emissão $\beta^{-}$pode se dar por mais de um ramo de desintegração, decaindo para diferentes níveis de energias de estados excitados dos núcleos filhos, que então se desexcitam por emissão gama e/ou elétrons de conversão interna. Sendo a energia da transição dividida entre a partícula $\beta^{-}$e o antineutrino de modo estatístico, a energia do $\beta^{-}$apresenta um espectro contínuo de energia que se estende de zero até um valor máximo permitido, e é correspondente à diferença entre a energia de 
desintegração e a energia do nível do estado excitado do núcleo fillho para o qual ele decaiu, ou seja ${ }^{[44]}$ :

$$
E_{\text {max }}=Q^{-}-E_{i}
$$

na qual

$\mathrm{Q}^{-}$é a energia de desintegração, igual à diferença de massa atômica, expressa em unidades de energia, entre os estados fundamentais do nuclídeo pai e filho.

$E_{i}$ é a energia do nível para o qual ocorre a desintegração.

A energia média de uma partícula $\beta^{-}$para uma determinada transição é dada por ${ }^{[43]}$.

$$
E_{\text {méd }}=\frac{\int_{0}^{E_{\text {máx }}} E N(E) d E}{\int_{0}^{E_{\text {máx }}} N(E) d E}
$$

$\mathrm{Na}$ qual $\mathrm{N}(\mathrm{E})$ é o número de partículas $\beta^{-}$com energia entre $\mathrm{E}$ e $\mathrm{E}+\mathrm{dE}$.

A figura 1.2 mostra um espectro típico de uma transição $\beta^{-}$permitida ${ }^{[44]}$.

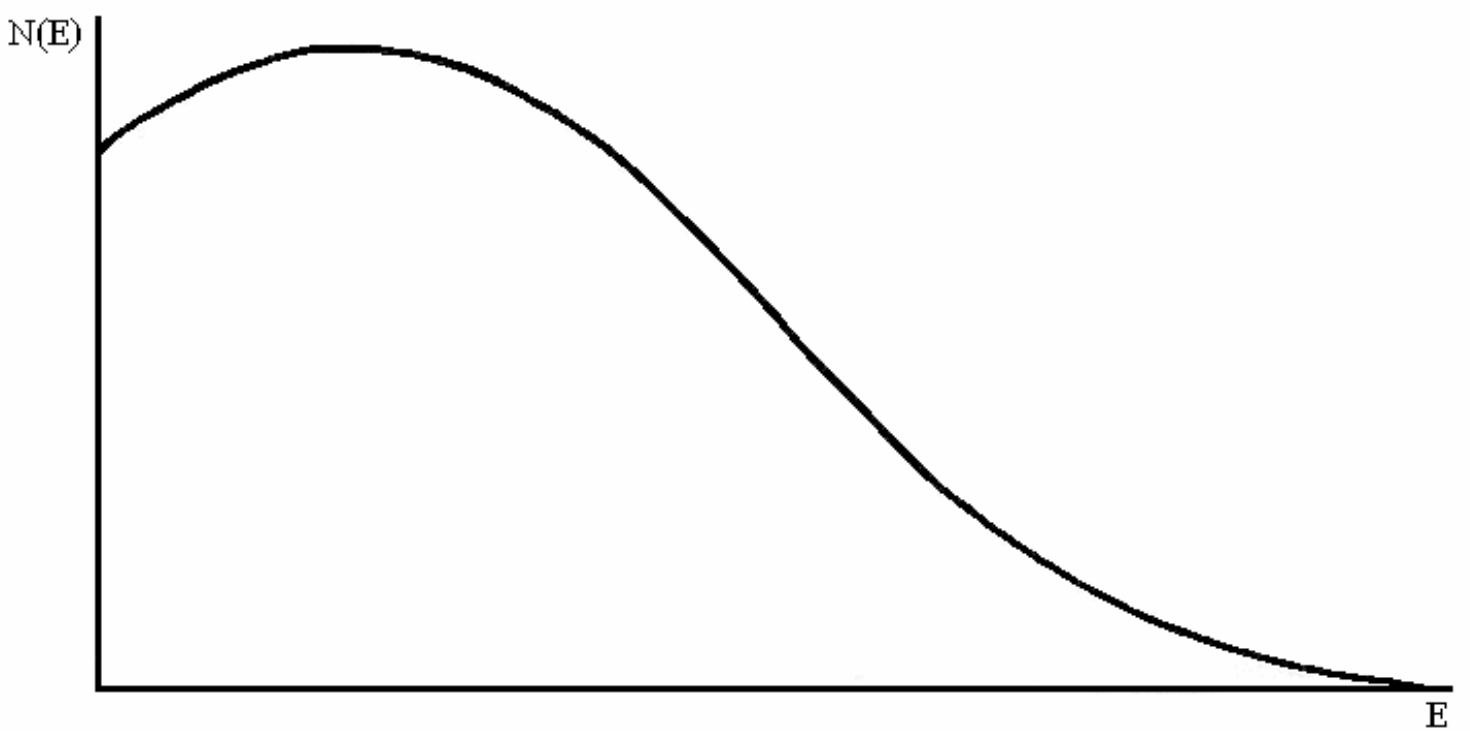

Figura 1.2 - Espectro típico de uma transição $\beta^{-}$permitida. 
- Desintegração $\beta^{+}$: Neste processo, analogamente ao processo anterior, um próton é convertido em um nêutron, e um elétron positivo $\beta^{+}$(pósitron) e um neutrino são emitidos do núcleo como resultado do processo ${ }^{[44]}$ :

$$
\mathrm{p} \rightarrow \mathrm{n}+\mathrm{e}^{+}+\mathrm{v}
$$

Esta desintegração diminui a carga nuclear de uma unidade.

Analogamente a emissão $\beta^{-}$, a desintegração $\beta^{+}$pode se dar por mais de um ramo de desintegração, e a energia do $\beta^{+}$também apresenta um espectro contínuo de energia que se estende de zero até um valor máximo ${ }^{[44]}$ :

$$
E_{\max }=Q^{+}-2 m_{0} c^{2}-E_{i}
$$

na qual

$\mathrm{Q}^{+} \quad$ é a energia de desintegração

$\mathrm{m}_{0} \mathrm{c}^{2}$ é a energia correspondente à massa de um elétron em repouso

$\mathrm{E}_{\mathrm{i}} \quad$ é a energia do nível para o qual ocorre a desintegração

A figura 1.3 mostra um espectro típico de uma transição $\beta^{+}$permitida ${ }^{[44]}$.

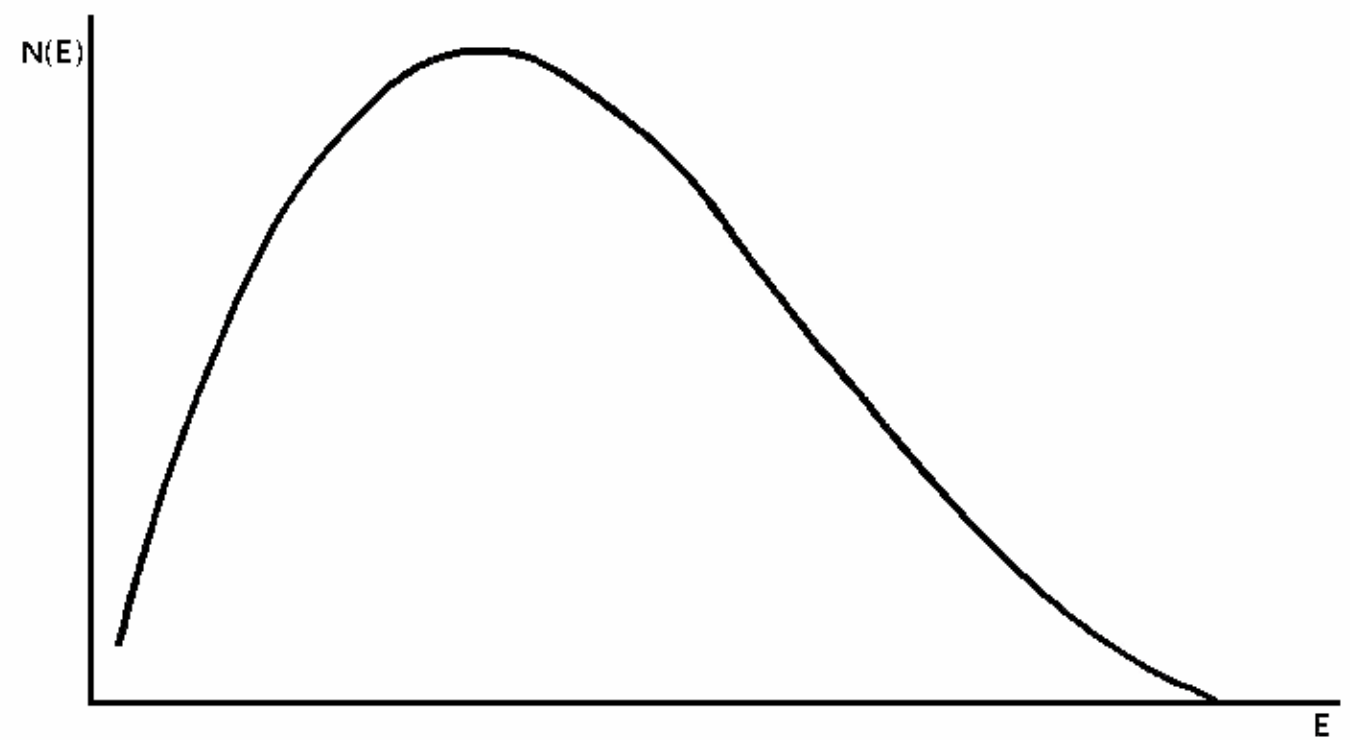

Figura 1.3 - Espectro típico de uma transição $\beta^{+}$permitida. 
A desintegração $\beta^{+}$concorre com o processo de captura eletrônica, mas requer uma energia limiar de $2 m_{0} c^{2}$ (ou 2 x $0,511 \mathrm{MeV}$ ) para que ocorra, ou seja ${ }^{[44]}$ :

$$
Q^{+}-E_{i}>2 m_{0} c^{2}
$$

\subsection{Captura Eletrônica (CE)}

Neste processo, um elétron orbital é capturado pelo núcleo induzindo à conversão de um próton em um nêutron e à emissão de um neutrino, o qual carrega a energia da transição ${ }^{[44]}$ :

$$
e^{-}+p \rightarrow n+v
$$

Nesta desintegração a carga nuclear diminui de uma unidade e a carga atômica permanece constante ${ }^{[44]}$.

$$
{ }_{Z}^{A} X+e^{-} \rightarrow{ }_{Z-1}^{A} Y+v
$$

Este processo deixa uma vacância na camada atômica que é preenchido por outro elétron atômico. Para desintegrações com energia maior que duas vezes a energia de ligação da camada $\mathrm{K}$, o elétron capturado pertence a uma camada $\mathrm{K}$ em $90 \%$ dos casos, e os $10 \%$ restantes de uma camada L ou maior. No processo de desexcitação do nuclídeo, o preenchimento da vacância deixado pelo processo de captura eletrônica é acompanhado da emissão de raios-X característicos ou elétrons Auger.

\subsection{Desexcitação de Estados do Núcleo Filho}

Uma transição pode ocorrer pela desexcitação de um núcleo atômico para um estado de menor energia, podendo ou não ser o estado fundamental. Ela manifesta-se espontaneamente pela emissão de um fóton gama ou pela transferência da energia 
excedente para um elétron da camada atômica, denominado elétron de conversão e, mais raramente quando condições de energia tornam possíveis, por um par elétron-pósitron.

A intensidade da transição gama é dada por ${ }^{[44]}$ :

$$
\mathrm{T}_{\gamma}=\mathrm{I}_{\gamma}+\mathrm{I}_{\mathrm{ec}}+\mathrm{I}_{\mathrm{e}^{ \pm}}
$$

na qual $\mathrm{I}_{\gamma}, \mathrm{I}_{\mathrm{ec}}$ e $\mathrm{I}_{\mathrm{e}^{ \pm}}$são as probabilidades de emissão gama, elétron de conversão ou par elétron-pósitron, respectivamente.

O terceiro processo não foi considerado no presente trabalho pois a intensidade para essa emissão é menor que $10^{-4}[44]$.

\subsubsection{Emissão $\gamma$}

A energia do raio gama emitido é dada por ${ }^{[44]}$ :

$$
E_{\gamma}=\left(E_{i}-E_{f}\right)-E_{r}
$$

na qual

$\mathrm{E}_{\mathrm{i}}-\mathrm{E}_{\mathrm{f}}$ é a diferença de energia entre os níveis inicial e final da transição gama.

$\mathrm{E}_{\mathrm{r}} \quad$ é a energia de recuo do estado final do núcleo, dado por ${ }^{[44]}$ :

$$
E_{r}=\frac{\left(E_{\gamma}\right)^{2}}{2 M_{N} c^{2}}
$$

na qual

$\mathrm{M}_{\mathrm{N}}$ é a massa do núcleo de recuo

c é a velocidade da luz

A energia de recuo é usualmente desprezível, exceto para altas energias gama e baixos números atômicos. Por exemplo, para a emissão gama de $1369 \mathrm{keV}$ do ${ }^{24} \mathrm{Na}$, 
$\mathrm{E}_{\mathrm{r}}=42 \mathrm{eV}$. Para conservação do momento, o núcleo recua em direção oposta à do raio $\gamma$ emitido.

Outra propriedade importante de um estado nuclear é sua meia-vida. Existe uma probabilidade $\lambda_{\mathrm{i}}$ associada a cada modo de desintegração de um nível. Se um estado excitado decai para vários níveis de menor energia, as probabilidades de transição associadas são todas independentes. A probabilidade total $\lambda$ será a soma dos valores individuais, ou seja ${ }^{[45]}$ :

$$
\lambda=\lambda_{1}+\lambda_{2}+\lambda_{3}+\cdots
$$

e a meia-vida do estado excitado é dada por ${ }^{[45]}$ :

$$
T_{\frac{1}{2}}=\frac{\ln 2}{\lambda}
$$

Há alguns estados excitados que tem vida-média relativamente longas. Este tipo de transição de um núcleo filho excitado para um nível de energia mais baixo do mesmo núcleo é chamada transição isomérica. O isomerismo nuclear é indicado pela letra m (de meta-estável) após o número de massa atômica do radionuclídeo. Na figura 1.4 é mostrado o esquema de desintegração do estado isomérico (ou meta-estável) do ${ }^{123 \mathrm{~m}} \mathrm{Te}^{[45]}$.

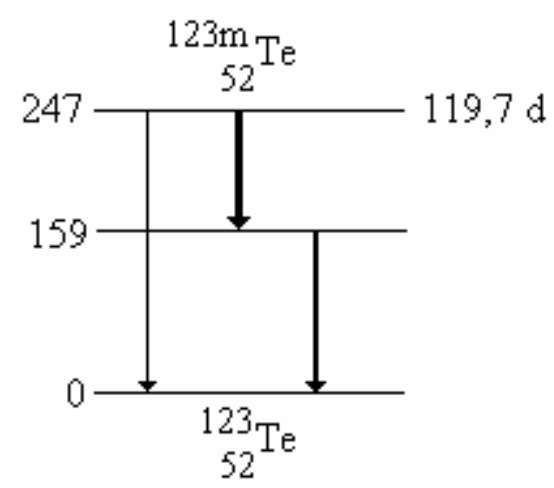

Figura 1.4 - Esquema de desintegração isomérico do ${ }^{123 \mathrm{~m}} \mathrm{Te}$ 


\subsubsection{Conversão Interna}

O processo de conversão interna inicia-se com o núcleo em estado excitado, que pode ter sido formado por um processo precedente - freqüentemente uma desintegração radioativa. A energia de desexcitação do núcleo é transferida diretamente a um elétron orbital (K, L etc.) do átomo o qual é ejetado deste, carregando uma energia dada por ${ }^{[44]}$ :

$$
\mathrm{E}_{\mathrm{ec}_{\mathrm{x}}}=\mathrm{E}_{\gamma}-\mathrm{E}_{\mathrm{x}}
$$

na qual $E_{X}$ é a energia de ligação do elétron na camada $X(X=K, L, M$...).

Diferentemente da desintegração $\beta$, os elétrons de conversão interna são monoenergéticos, tendo energias um pouco menores, com relação aos fótons gama com os quais concorrem, na faixa de energias entre $\mathrm{keV}$ a alguns $\mathrm{MeV}$.

Para uma dada transição, a razão entre as da probabilidades de emissão do elétron de conversão da camada $\mathrm{K}$ e de emissão do raio- $\gamma$ é chamada de coeficiente de conversão interna para a camada $\mathrm{K}$, sendo definido por $^{[44]}$ :

$$
\alpha_{K}=\frac{I_{e c_{K}}}{I_{\gamma}}
$$

na qual $I_{e c_{K}}$ e $I_{\gamma}$ são as probabilidades de emissão dos elétrons de conversão $\mathrm{K}$ e da radiação gama, respectivamente.

Os coeficientes de conversão interna para as outras camadas são definidos de modo análogo:

$$
\alpha_{\mathrm{L}}=\frac{\mathrm{I}_{\mathrm{ec}}}{\mathrm{I} \gamma}, \quad \alpha_{\mathrm{L}_{\mathrm{i}}}=\frac{\mathrm{I}_{\mathrm{ec}_{\mathrm{L}_{\mathrm{i}}}}}{\mathrm{I}_{\gamma}} \quad(\mathrm{i}=1,2,3)
$$


na qual $\alpha_{\mathrm{L}}=\alpha_{\mathrm{L}_{1}}+\alpha_{\mathrm{L}_{2}}+\alpha_{\mathrm{L}_{3}}$

$$
\alpha_{M_{i}}=\frac{I_{e c_{M_{i}}}}{I_{\gamma}} \quad(i=1,2,3 \ldots)
$$

O coeficiente de conversão interna total é igual à soma dos coeficientes das várias camadas ou subcamadas atômicas, e é dado por:

$$
\alpha_{t}=\alpha_{K}+\alpha_{L}+\alpha_{M}+\cdots=\frac{I_{e c}}{I_{\gamma}}
$$

e $\mathrm{I}_{\mathrm{ec}}$ é a probabilidade total para emissão de elétrons de conversão da transição em questão.

A partir destas definições, tem-se as seguintes relações:

$$
\begin{gathered}
I_{\gamma}=\frac{1}{1+\alpha_{t}} \\
I_{e c}=\frac{\alpha_{t}}{1+\alpha_{t}} \cdot T_{\gamma} \\
I_{e c_{K}}=\frac{\alpha_{K}}{1+\alpha_{t}} \cdot T_{\gamma}
\end{gathered}
$$

na qual $\mathrm{T}_{\gamma}$ é a probabilidade total da transição, $\mathrm{T}_{\gamma}=\mathrm{I}_{\gamma}+\mathrm{I}_{\mathrm{ec}}$.

Encontra-se com freqüência na literatura os valores dos coeficientes de conversão interna relativos, representados simbolicamente pelas razões $\frac{\mathrm{K}}{\mathrm{L}}, \frac{\mathrm{K}}{\mathrm{LM}}, \frac{\mathrm{K}}{\mathrm{LM} \ldots}$ que são definidas da seguinte maneira: 


$$
\begin{gathered}
\frac{K}{L}=\frac{I_{e c_{K}}}{I_{e c_{L}}}=\frac{\alpha_{K}}{\alpha_{L}} \\
\frac{K}{L M}=\frac{I_{e c_{K}}}{I_{e c_{L}}+I_{e c_{M}}+\cdots}=\frac{\alpha_{K}}{\alpha_{L}+\alpha_{M}+\cdots}
\end{gathered}
$$

O processo de conversão interna depende do estado inicial do elétron (camada atômica ou subcamada), do número atômico $\mathrm{Z}$ e da transição nuclear.

Um exemplo de um espectro de um elétron de conversão é mostrado na figura

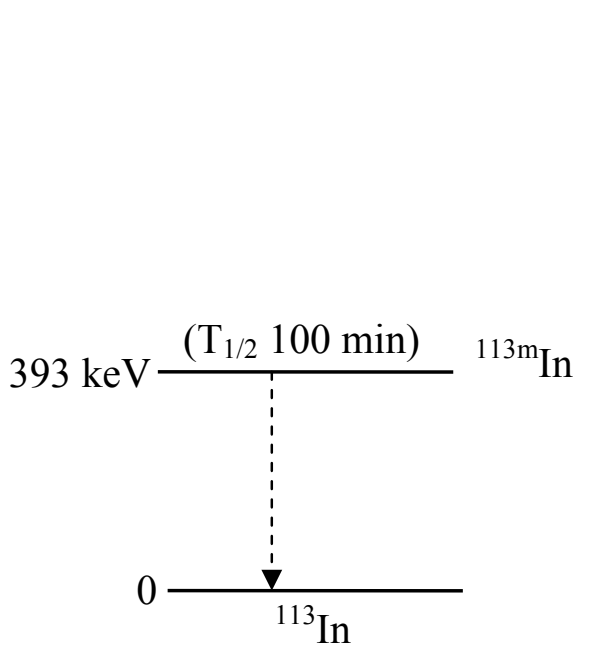
$1.5^{[42]}$.

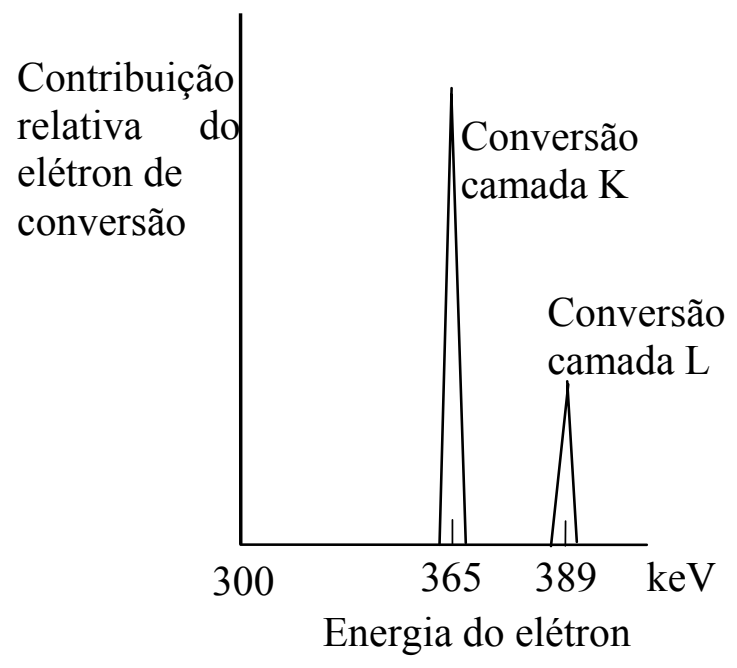

Figura 1.5 - Espectro do elétron de conversão da conversão interna do nível isomérico para energia de $393 \mathrm{keV}$ do ${ }^{113 \mathrm{~m}} \mathrm{In}$.

\subsection{Rearranjo Eletrônico}

Os processos de captura eletrônica e conversão interna induzem o átomo a um estado excitado, que dão origem a vacâncias nos orbitais eletrônicos. A tendência natural é haver um rearranjo dos elétrons nas camadas com o intuito de preencher essas vacâncias. $\mathrm{O}$ preenchimento de uma vacância é acompanhado pela emissão de raio-X ou de um elétron Auger, criando novas vacâncias nas camadas eletrônicas menos ligadas. A vacância inicial é assim transferida por cascata para as camadas periféricas. A energia liberada corresponde à energia de ligação do elétron na camada no qual a vacância inicial foi produzida. 


\section{Vacância na camada $K$}

O número de vacâncias $\mathrm{K}$ criadas durante uma transição por captura eletrônica com probabilidade $\mathrm{I}_{\varepsilon}$ corresponde a $\mathrm{I}_{\varepsilon} \mathrm{P}_{\mathrm{K}}$.

Para um dado radionuclídeo, o número total de vacâncias criadas na camada K, considerando eventos de captura eletrônica e conversão interna, é dado por $^{[44]}$.

$$
n_{K}=\sum I_{\varepsilon} P_{K}+\sum T_{\gamma} \frac{\alpha_{K}}{1+\alpha_{t}}
$$

Fluorescência produzida pela camada K

A probabilidade de que o preenchimento de uma vacância na camada $\mathrm{K}$ seja acompanhado por radiação $\mathrm{X}_{\mathrm{K}}$ (fluorescência) é dada por ${ }^{[44]}$ :

$$
\omega_{K}=\frac{I_{X_{K}}}{n_{K}}
$$

na qual $I_{X_{K}}$ é a probabilidade de emissão de fótons $X_{K}$.

A probabilidade de emissão de elétron Auger é deduzida a partir da probabilidade de fluorescência:

$$
a_{K}=1-\omega_{K}
$$

Deste modo, obtém-se a probabilidade de emissão de étrons Auger K: 


$$
I_{e A_{K}}=a_{K} n_{K}
$$

A probabilidade de fluorescência $\omega_{\mathrm{K}}$ depende exclusivamente do número atômico $\mathrm{Z}$, exceto para átomos de baixo $\mathrm{Z}$, para os quais ela pode ser influenciada pelo estado químico.

Para radionuclídeos com baixos números atômicos temos $\omega_{\mathrm{K}}<<1$. Este parâmetro aproxima-se de 1 para radionuclídeos com números atômicos elevados.

\section{Raios $X$}

Raios X são radiações eletromagnéticas emitidas em transições dos elétrons atômicos entre diferentes estados no átomo. As emissões ocorrem quando os elétrons mais externos preenchem as vacâncias deixadas nas camadas mais internas. Cada transição possui uma energia característica, dada pela diferença entre as energias dos estados inicial e final. Quando a vacância é na camada $K$, se dá a emissão de fóton $X_{K}$; quando é na camada $\mathrm{L}$ ocorre a emissão de fóton $\mathrm{X}_{\mathrm{L}}$, e assim sucessivamente.

A energia do raio $X_{K}$ emitido para uma transição $K X(X=L, M, N, \ldots)$ é dada por:

$$
E_{X_{K}}=E_{K}-E_{X}
$$

Onde $E_{K}$ e $E_{X}$ são as energias de ligação dos elétrons nas camadas $\mathrm{K}$ e $\mathrm{X}$, respectivamente.

\section{Elétrons Auger}

Em alguns casos, a energia de excitação do átomo é transferida para um elétron de uma camada mais externa, provocando a sua ejeção do átomo. Este elétron terá uma energia dada pela diferença entre as energias de excitação do átomo e de ligação do elétron ejetado. Em geral, essa energia é menor que a de partículas $\beta$ ou elétrons de conversão 
interna, uma vez que a emissão de elétron Auger ocorre com maior probabilidade em radionuclídeos de $\mathrm{Z}$ baixo $(\mathrm{Z}<45)$, por apresentarem baixas energias de ligação dos elétrons.

Quando a vacância ocorre na camada K a energia do elétron Auger-K é dada $\operatorname{por}^{[44]}$ :

$$
E_{A_{K}}=E_{K}-E_{X}-E_{Y}-\Delta E \quad\left(E_{Y} \leq E_{X}\right)
$$

$\mathrm{Na}$ qual $\mathrm{E}_{\mathrm{K}}, \mathrm{E}_{\mathrm{X}}, \mathrm{E}_{\mathrm{Y}}$, são as energias de ligação dos elétrons nas camadas $\mathrm{K}, \mathrm{X}$ e $\mathrm{Y}$ (ou subcamadas), respectivamente. $\Delta \mathrm{E}$ é um termo corretivo para o caso em que a energia de ligação do elétron do átomo no estado excitado seja muito maior do que a do átomo em seu estado fundamental.

\subsection{Interação da Radiação com a Matéria}

Quando partículas carregadas atravessam um meio, eles sofrem colisões elásticas e inelásticas com os átomos e moléculas deste meio. A colisão elástica produz apenas deflexões e a inelástica produz também um íon positivo e um elétron, usualmente chamado de par-de-íons. Em média, aproximadamente $35 \mathrm{eV}$ de energia é necessária para formar cada par-de-íons em gases. A produção dos pares-de-íons por partículas carregadas se dá por interações Coulombianas.

Outras formas de transferência de energia podem ocorrer por meio de excitação dos átomos ou moléculas do meio e pela produção de radiação de freiamento ("Bremsstrahlung”). Esta última ocorre preferencialmente pela interação de elétrons ou pósitrons com a matéria e é dominante em altas energias e materiais de alto número atômico.

Grande parte das fontes radioativas gama emissoras decaem pela emissão de radiação beta. Por esta razão, radiação secundária na forma de raio-X (“Bremsstrahlung”) pode ser gerada e contribuir para o espectro medido. 
A energia dos fótons $\mathrm{X}$ ou gama pode ser expressa como $\mathrm{E}=\mathrm{h} v$, e as interações desses fótons com a matéria são consideradas não dependentes da origem do fóton, dependendo apenas de sua energia e do material considerado.

Diferentemente das partículas carregadas, um feixe de radiação gama, bem colimado, apresenta uma atenuação exponencial na matéria. Isto ocorre porque os fótons são absorvidos ou espalhados em um único evento. Há diversos tipos de interação que causam espalhamento ou absorção de raios gama:

Tipo de interação

1- Interação com elétrons atômicos

2- Interação com nucleons

3- Interação com o campo elétrico ao redor do núcleo ou elétron

4- Interação com o campo nuclear ao redor dos nucleons

Há 12 maneiras de combinar a coluna 1 e 2; assim em teoria há 12 processos diferentes para cada raio gama poder ser absorvido ou espalhado. Vários desses processos são pouco freqüentes. Os processos principais de transferência de energia pelos raios $\mathrm{X}$ e gama na matéria são: (i) efeito Fotoelétrico, importante em baixas energias; (ii) efeito Compton, importante para energias intermediárias; e (iii) Produção-de-Pares (elétronpósitron) que ocorre para radiação gama com energias acima de $1022,0 \mathrm{keV}^{[41]}$.

\section{Seção de Choque}

A base de todas as medidas de absorção de raios $\mathrm{X}$ e raios gama é o fato de que a intensidade de radiação decresce ao passar através da matéria. Em tal passagem, para uma pequena espessura $\Delta \mathrm{x}$, a variação na intensidade $\Delta \mathrm{I}$ é proporcional à espessura e a intensidade incidente I, isto é, 


$$
\Delta \mathrm{I}=-\mu \mathrm{I} \Delta \mathrm{x}
$$

na qual $\mu$ é a constante de proporcionalidade, dependente do material e da energia do fóton, e é chamada de coeficiente de absorção ou seção de choque macroscópica. Se a radiação é homogênea, $\mu$ é constante, e a integração dessa equação fornece:

$$
\frac{\mathrm{I}}{\mathrm{I}_{0}}=\mathrm{e}^{-\mu \mathrm{x}}
$$

A equação fornece a intensidade de radiação I após uma partícula de intensidade inicial $\mathbf{I}_{\mathbf{0}}$ ter atravessado uma espessura $\mathbf{x}$ de um dado material.

Os três processos de interação de radiação gama com a matéria (efeito Compton, efeito Fotoelétrico, e Produção-de-Pares) agem independentemente um do outro, assim, podemos separar o coeficiente de absorção em três partes que designaremos por $\sigma$ para efeito Compton, $\tau$ para o efeito fotoelétrico, e $\kappa$ para a produção de pares:

$$
\mu=\sigma+\tau+\kappa
$$

Um quarto processo que é o espalhamento coerente $\left(\sigma_{\text {coe }}\right)$ também pode contribuir para atenuação do feixe de fótons, embora não transfira energia aos átomos do meio:

$$
\mu=\sigma+\tau+\kappa+\sigma_{\text {coe }}
$$

\subsubsection{Efeito Fotoelétrico}

Para energias abaixo de aproximadamente $100 \mathrm{keV}$, o modo predominante de interação de fótons gama ou $\mathrm{X}$ em materiais de alto $\mathrm{Z}$ é o efeito fotoelétrico. No efeito fotoelétrico, a energia total do fóton hv é transferida para um elétron orbital, o qual abandona o átomo com uma energia igual à do fóton, menos a energia de ligação $\left(\mathrm{E}_{\mathrm{b}}\right) \mathrm{da}$ camada K, L, M, etc., do qual é ejetado: 


$$
\mathrm{E}_{\mathrm{e}^{-}}=\mathrm{h} v-\mathrm{E}_{\mathrm{b}}
$$

na qual $\mathrm{E}_{\mathrm{e}^{-}}$é a energia cinética do elétron ejetado. O restante da energia aparece na forma de raio X característico ou elétrons Auger, durante o preenchimento da vacância na camada interna. A figura 1.6 ilustra o processo.

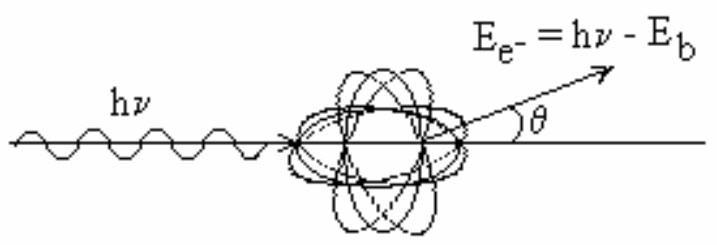

Figura 1.6 - Efeito Fotoelétrico.

Um fóton incidente não pode ser totalmente absorvido por um elétron livre. A absorção total somente pode ter lugar se o elétron estiver ligado ao átomo. Desse modo, o momento é conservado pelo recuo do átomo residual inteiro. Como podem ser esperados, os elétrons mais ligados tem a maior probabilidade de absorção por efeito fotoelétrico, sendo que $80 \%$ dos processos de absorção fotoelétrica ocorrem na camada $\mathrm{K}$, desde que a energia do fóton incidente $h v$ exceda a energia de ligação correspondente, e uma grande parte dos $20 \%$ restantes ocorrem na camada L. O efeito fotoelétrico possui uma forte dependência com o número atômico $\left(Z^{3}\right.$ ou $\left.Z^{4}\right)$ e cai com $\frac{1}{(h v)^{3}}$. Portanto, é um processo dominante em baixas energias.

A absorção fotoelétrica é o processo ideal se estamos interessado em medir a energia do raio gama original. Como a energia dissipada por emissão de raios $\mathrm{X}$ característicos e elétrons Auger normalmente é absorvida no meio, um único pico aparecerá no espectro de energia absorvida, correspondendo a energia dos raios gama incidentes (figura 1.7). 


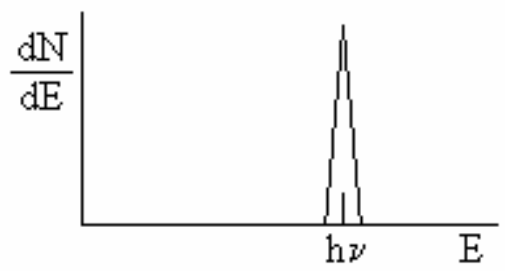

Figura 1.7 - Espectro de energia correspondente à absorção total de um feixe de radiação gama ou X monoenergético ${ }^{[42]}$.

\subsubsection{Efeito Compton}

No efeito Compton, o fóton é espalhado e comporta-se como uma partícula, com energia, hv, e momento $\frac{h v}{c}$. A energia é transferida para um elétron livre que é defletido em uma nova direção, sendo o fóton espalhado com energia menor. A divisão da energia entre os dois depende do ângulo de espalhamento (figura 1.8).

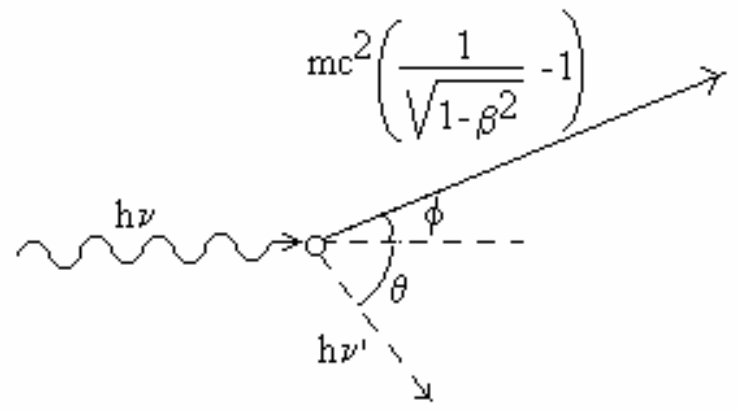

Figura 1.8 - Espalhamento Compton

A energia do raio gama espalhado hv' em termos do ângulo de espalhamento $\theta$ é dada por ${ }^{[40]}$ :

$$
h v^{\prime}=\frac{h v}{1+\alpha(1-\cos \theta)}
$$

na qual $\alpha=\frac{h v}{m_{0} c^{2}}$. 
A energia cinética do elétron de recuo é então ${ }^{[40]}$ :

$$
\mathrm{E}_{\mathrm{e}^{-}}=\mathrm{h} v-\mathrm{h} v^{\prime}=\mathrm{h} v\left(1-\frac{1}{1+\alpha(1-\cos \theta)}\right)
$$

Podemos deduzir que para ângulos de espalhamento muito pequenos, tem-se $\theta \cong 0, \mathrm{~h} v \cong \mathrm{h} v$ e $\mathrm{E}_{\mathrm{e}^{-}} \cong 0$. Neste caso, elétron de recuo Compton tem energia muito baixa e o raio gama espalhado tem aproximadamente a mesma energia do raio gama incidente. Para uma colisão frontal, ou seja $\theta=\pi$, o raio gama incidente é retro-espalhado voltando para sua direção de origem, enquanto o elétron de recuo tem direção incidente. Essa situação corresponde à energia máxima que pode ser transferida para um elétron em uma interação Compton.

A seção de choque para o número de fótons espalhados por elétron e por unidade de ângulo sólido na direção $\theta$, é dada por ${ }^{[46]}$ :

$$
\frac{d_{e} \sigma(\theta)}{d \Omega}=\frac{r_{o}{ }^{2}}{2}\left\{\frac{1}{[1+\alpha(1-\cos \theta)]^{2}}\left[1+\cos ^{2} \theta+\frac{\alpha^{2}(1-\cos \theta)^{2}}{[1+\alpha(1-\cos \theta)]}\right]\right\}
$$

Aqui $\alpha=\frac{\mathrm{h} v}{\mathrm{mc}^{2}}$ e $\mathrm{r}_{\mathrm{o}}=\frac{\mathrm{e}^{2}}{\mathrm{mc}^{2}}=2,818 \cdot 10^{-13} \mathrm{~cm}$.

A seção de choque para o total da energia espalhada por elétron e por unidade de ângulo sólido é dada por ${ }^{[46]}$ :

$$
\frac{d_{e} \sigma_{s}(\theta)}{d \Omega}=\frac{r_{o}{ }^{2}}{2}\left\{\frac{1}{[1+\alpha(1-\cos \theta)]^{3}}\left[1+\cos ^{2} \theta+\frac{\alpha^{2}(1-\cos \theta)^{2}}{[1+\alpha(1-\cos \theta)]}\right]\right\}
$$

A seção de choque total Compton ${ }_{e} \sigma$ é obtida pela integração da equação (1.40) no intervalo $[0, \pi]$ para $\theta$ e $[0,2 \pi]$ para $\phi^{[46]}$ : 


$$
\mathrm{e}^{\sigma}=2 \pi \mathrm{r}_{\mathrm{o}}^{2}\left\{\frac{1+\alpha}{\alpha^{2}}\left[\frac{2(1+\alpha)}{1+2 \alpha}-\frac{1}{\alpha} \ln (1+2 \alpha)\right]+\frac{1}{2 \alpha} \ln (1+2 \alpha)-\frac{1+3 \alpha}{(1+2 \alpha)^{2}}\right\}
$$

Integrando a equação (1.41) entre os mesmos limites obtemos o coeficiente de espalhamento Compton, e $\sigma_{\mathrm{s}}$, dado por ${ }^{[46]}$ :

$$
\mathrm{e}^{\sigma_{\mathrm{S}}}=\pi \mathrm{r}_{\mathrm{O}}{ }^{2}\left[\frac{1}{\alpha^{3}} \ln (1+2 \alpha)+\frac{2(1+\alpha)\left(2 \alpha^{2}-2 \alpha-1\right)}{\alpha^{2}(1+2 \alpha)^{2}}+\frac{8 \alpha^{2}}{3(1+2 \alpha)^{3}}\right]
$$

As relações acima indicam que o espalhamento Compton é dominante em energias intermediárias de fótons $\mathrm{X}$ ou gama. Por outro lado, este processo está relacionado com a densidade de elétrons no meio. Como esta densidade é aproximadamente constante em diferentes materiais, resulta que a probabilidade de interação por efeito Compton praticamente independe do material absorvedor.

\subsubsection{Produção-de-Pares}

Para um fóton incidente de energia maior do que duas vezes a energia de repouso do elétron, ou seja, maior do que $2 m_{0} c^{2}=1022,0 \mathrm{keV}$, um terceiro tipo de interação conhecida como produção-de-pares, torna-se importante. Nesta interação o fóton é completamente absorvido e em seu lugar aparece um par elétron-pósitron (figura 1.9).

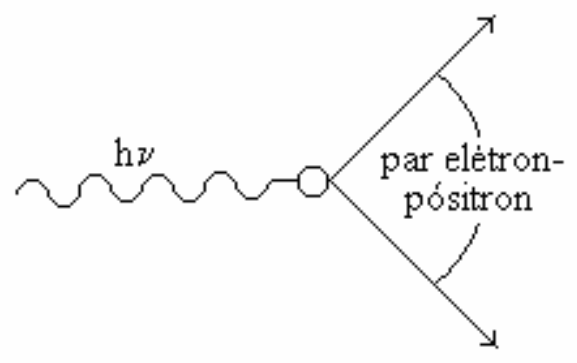

Figura 1.9 - Efeito de Produção de Pares. 
O processo ocorre somente no campo de partículas carregadas, principalmente dentro do campo Coulombiano de um núcleo. A energia em excesso, acima de 1022,0 keV, é transferida na forma de energia cinética das partículas ${ }^{[42]}$ :

$$
\mathrm{E}_{\mathrm{e}^{-}}+\mathrm{E}_{\mathrm{e}^{+}}=\mathrm{hv}-2 \mathrm{~m}_{\mathrm{o}} \mathrm{c}^{2}
$$

O coeficiente de produção-de-pares interno é definido por ${ }^{[44]}$ :

$$
\alpha_{\pi}=\frac{\mathrm{I}\left(\mathrm{e}^{ \pm}\right)}{\mathrm{I}_{\gamma}}
$$

A intensidade desta emissão é em geral baixa e $\alpha_{\pi}$ é geralmente menor do que $10^{-4}$. Para energias típicas o elétron e o pósitron, penetram no máximo poucos milímetros em material sólido antes de perder toda a energia cinética no meio absorvedor.

Na figura 1.10 temos a representação esquemática dos estados de energia positivo e negativo do elétron. Pares elétron-pósitron são criados pelas transições que somam uma energia maior do que $2 \mathrm{~m}_{0} \mathrm{c}^{2}$ para um elétron originalmente em um estado de energia negativa. A lacuna é então um pósitron de energia total $\mathrm{m}_{\mathrm{o}} \mathrm{c}^{2}+\mathrm{E}_{\mathrm{e}}+$, e o estado excitado é simplesmente um elétron de energia total $\mathrm{m}_{\mathrm{o}} \mathrm{c}^{2}+\mathrm{E}_{\mathrm{e}^{-}}$.

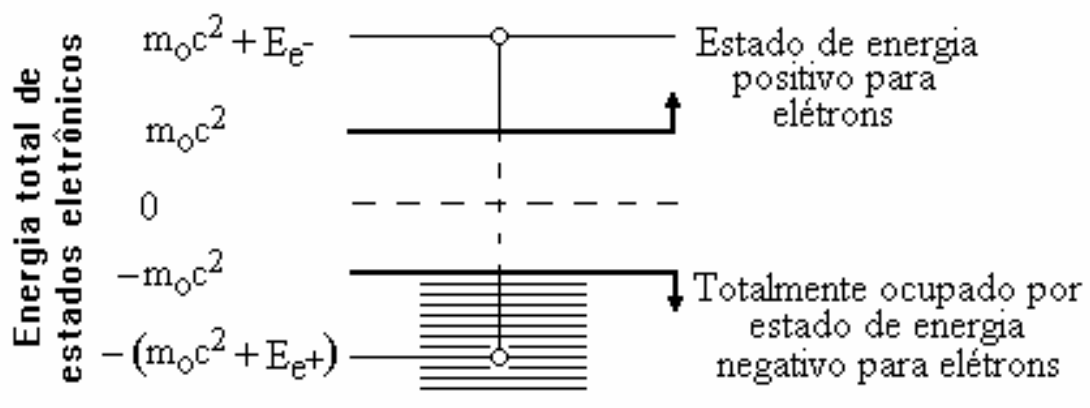

Figura 1.10 - Representação esquemática dos estados de energia positivo e negativo do elétron ${ }^{[40]}$.

O gráfico da energia cinética total das partículas carregadas (elétron-pósitron) criado pelo raio gama incidente está localizado $2 \mathrm{~m}_{0} \mathrm{c}^{2}$ abaixo da energia do raio gama 
incidente, como ilustrado na figura 1.11. Este espectro corresponde ao pico de duplo escape dos gamas de aniquilação.

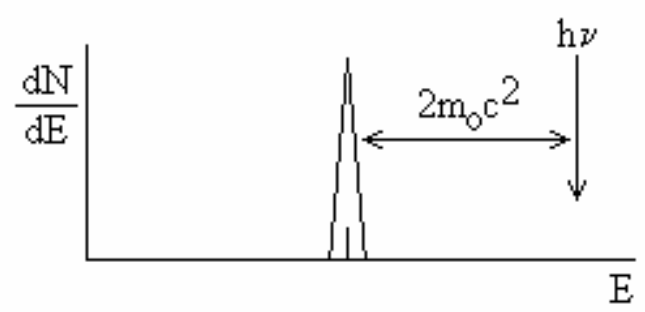

Figura 1.11 - Gráfico da energia cinética total das partículas carregadas (elétron-pósitron) $)^{[42]}$.

O pósitron subsequentemente aniquila-se com um elétron produzindo dois fótons de $0,511 \mathrm{MeV}$, ditos fótons de aniquilação. Os processos de produção de pares e aniquilação normalmente ocorrem dentro do tempo de resolução do detector, e os fótons de aniquilação propagam-se essencialmente em direções opostas, um em relação ao outro.

A detecção de radiação gama é possível pelas interações com a matéria dos elétrons secundários produzidos através dos processos descritos acima. Os raios gama, produzem elétrons dentro do volume sensível dos detectores, tais como cristais de Iodeto de Sódio, germânio e silício. No germânio e silício as interações produzem pares elétronlacuna, que são coletados e dão origem a pulsos, cuja amplitude está relacionada com a energia depositada no cristal.

A importância relativa dos três processos descritos acima para diferentes materiais absorvedor e energias de raio gama são ilustrados na figura 1.12. A linha a esquerda representa a energia para o qual absorção fotoelétrica e espalhamento Compton são igualmente prováveis como uma função do número atômico do absorvedor. A linha à direita representa a energia para o qual o espalhamento Compton e produção-de-pares são igualmente prováveis. Três áreas estão assim definidas no gráfico dentro do qual absorção fotoelétrica, espalhamento Compton, e produção-de-pares cada um predominam. 


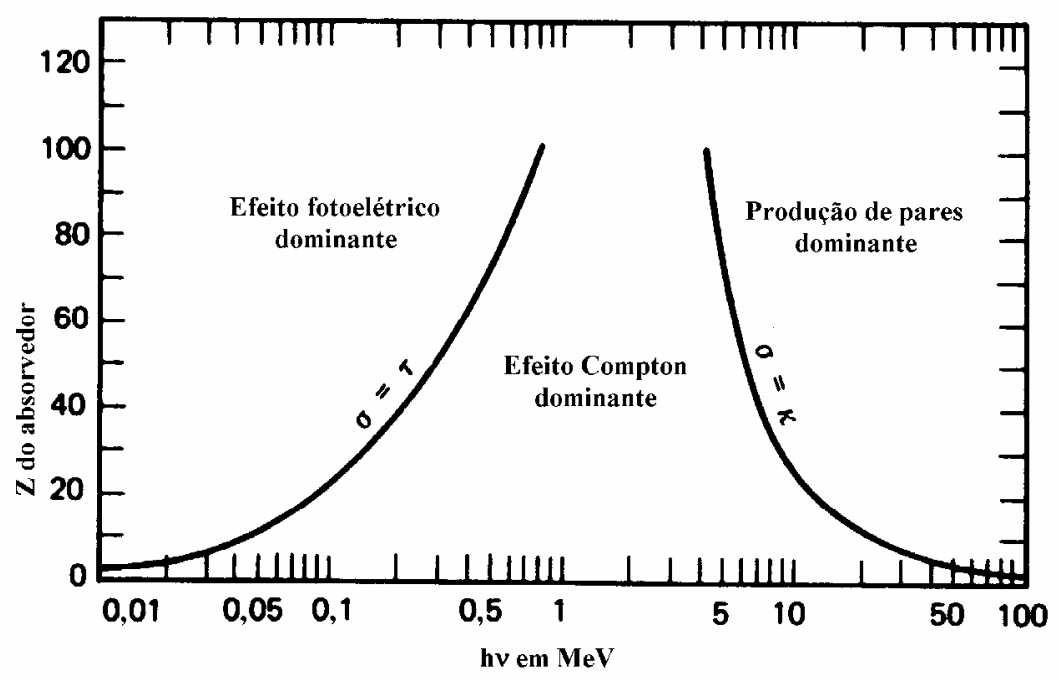

Figura 1.12 - Importância relativa dos três principais tipos de interação de raios gama ${ }^{[42]}$

\subsection{Espectrometria $\gamma$ e $X$}

A espectrometria $\gamma$ e X é um método de análise utilizada em metrologia das radiações ionizantes, e consiste basicamente em identificar e determinar a natureza e a atividade de radionuclídeos contidos em uma amostra, bem como calcular os parâmetros da emissão radioativa, a partir do registro e análise de seu espectro de emissão.

Este espectro é único para cada nuclídeo, caracterizado pelo tipo de emissão radioativa, energia e probabilidade de emissão da radiação. Para obtê-lo, utiliza-se um detector acoplado a um sistema eletrônico de aquisição de dados, resultando em um espectro de natureza discreta (linhas).

Diferentes fenômenos eletrônicos no sistema de medição provocam o alargamento dessas linhas, transformando-as em picos de distribuição centrados na energia da radiação. A largura a meia-altura destes picos (FWHM) é definida como resolução e permite avaliar a qualidade do dispositivo de detecção.

Para associar os picos de um espectro a um radionuclídeo, determina-se a relação energia-canal, por meio de uma calibração. A seguir, determina-se a curva de eficiência de absorção total, com o intuito de calcular a atividade dos radionuclídeo através 
das áreas sob os picos. A relação entre a área de um pico de energia $\mathrm{E}_{\gamma, \mathrm{X}}$ e a atividade é dada por:

$$
A=\frac{S\left(E_{\gamma, X}\right)}{\varepsilon\left(E_{\gamma, X}\right) p\left(E_{\gamma, X}\right) T}
$$

na qual A é a atividade da amostra, $\mathrm{S}\left(\mathrm{E}_{\gamma, \mathrm{X}}\right)$ é a área do pico de energia $\mathrm{E}_{\gamma, \mathrm{X}}$, corrigida para desintegração, radiação de fundo, tempo morto, efeito soma em cascata, atenuação e geometria, $\mathrm{p}\left(\mathrm{E}_{\gamma, \mathrm{X}}\right)$ é a probabilidade de emissão de radiação gama ou $\mathrm{X}$ de energia $\mathrm{E}_{\gamma, \mathrm{X}}, \varepsilon$ é a eficiência do pico de absorção total para esta energia, e T o tempo de medida.

\section{Formação de espectros}

Ao observar um espectro de emissão de fótons em um detector de radiação, é possível ver o resultado das diferentes interações produzidas por eles no interior do detector ou em materiais ao seu redor, tais como picos de absorção total, espalhamento Compton, fenômenos de escape e coincidências.

Picos de absorção total

Espectros de fótons que não sofreram interações com o meio exterior, depositando toda sua energia no interior do detector, por efeito Fotoelétrico, por várias interações Compton ou produção-de-pares seguida de Compton e Fotoelétrico, cuja energia depositada corresponda a energia específica do fóton.

\section{Espalhamento Compton}

Compõem espectros de formas diferentes:

a) Fótons que sofreram somente uma interação Compton no interior do detector. O fóton espalhado terá energia máxima quando o ângulo de espalhamento for zero e mínima quando o ângulo de espalhamento tender a $180^{\circ}$, portanto, a energia depositada no detector será máxima para ângulo de espalhamento de $180^{\circ}$ e zero para ângulo zero, 
resultando num fundo contínuo no espectro com energias entre zero $\mathrm{keV}$ e a energia máxima depositada.

b) Fótons que sofrem várias interações Compton no interior do detector antes de ser espalhado para fora dele. No espectro aparecem superpostos ao fundo contínuo e também além dele, formando um vale que acaba antes do pico de absorção total.

c) Fótons que interagem por efeito Compton com o meio externo antes de depositar sua energia no detector, encontrados no fundo contínuo e no vale. Em alguns casos, os fótons espalham-se no meio externo em um ângulo próximo a $180^{\circ}$ antes de atingir o detector, dando origem a um pico característico, chamado pico de retroespalhamento.

Fenômenos de escape

Quando um fóton com energia E maior que $1022 \mathrm{keV}$ alcança o detector, ele pode produzir um par elétron-pósitron acompanhado da aniquilação do pósitron dentro do detector e a emissão de dois fótons de $511 \mathrm{keV}$. Estes fótons podem sair do detector sem interagir, originando dois picos: o pico de escape simples, com energia de $511 \mathrm{keV}$ abaixo do pico de absorção total, quando um único fóton escapa, e o pico de duplo escape, com energia de $1022 \mathrm{keV}$ abaixo, se os dois fótons escapam. A produção-de-pares pode também ter origem no exterior do detector, sendo que, neste caso, um dos dois fótons de aniquilação pode interagir com o detector e dar origem a um pico com $511 \mathrm{keV}$, chamado pico de aniquilação.

Um outro fenômeno de escape está ligado aos fótons $\mathrm{X}$ de fluorescência. Neste caso, haverá um pico com energia $\mathrm{E}-\mathrm{E}_{\mathrm{X}}$, sendo $\mathrm{E}_{\mathrm{X}}$ a diferença entre a energia de ligação das duas camadas.

\section{Eventos em Coincidência}

A desexcitação do núcleo, sucedendo uma desintegração, pode originar uma ou várias transições gama. Quando emitidos em cascata, em um intervalo de tempo muito inferior ao tempo de coleta de cargas do detector, um único pulso, soma dos dois eventos distintos, será registrado, reduzindo a intensidade dos picos correspondentes à energia de 
cada fóton e originando um pico-soma de coincidência gama-gama. Pode ocorrer também, no caso de um fóton gama seguido de um fóton $\mathrm{X}$ de rearranjo, formando um pico soma por coincidência gama-X. 


\section{METODOLOGIA}

\subsection{Medida Absoluta da Atividade Pelo Método de Coincidência}

Os métodos absolutos, ou diretos, são aqueles na qual a taxa de desintegração pode ser determinada sem o conhecimento prévio dos parâmetros do detector (ângulo sólido, eficiência etc.) ou do valor de uma grandeza auxiliar, a não ser o tempo.

Um dos métodos de medida absolutos amplamente utilizado na padronização de radionuclídeos é o método de coincidência ${ }^{[1-7]}$, que possibilita a obtenção de resultados de taxa de desintegração com grande exatidão por meio das taxas de contagens, sem o conhecimento dos parâmetros inerentes ao processo de desintegração ou do equipamento utilizado.

\subsubsection{Princípio do Método}

O método de coincidência é utilizado para as padronizações de radionuclídeos que se desintegram pela emissão de duas radiações simultâneas e de natureza diferente, como $\alpha-\gamma, \beta-\gamma$, CE- $\gamma$. Baseia-se no uso de dois detectores estáveis no tempo, um para cada tipo de radiação emitida e um módulo para a determinação dos eventos coincidentes, isto é, detectados simultaneamente nos dois detectores, figura 2.1.

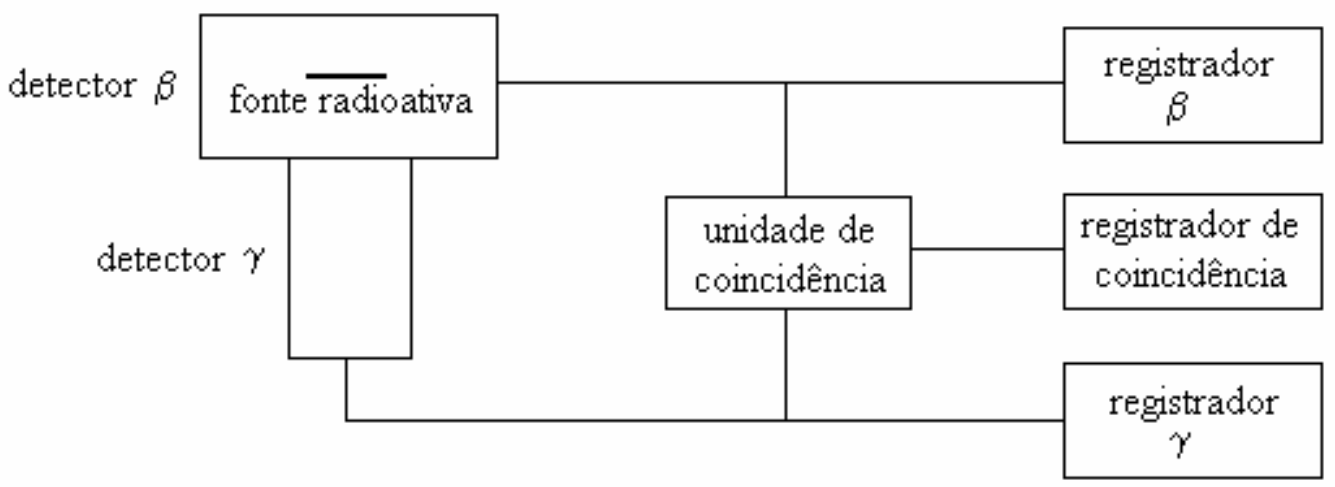

Figura 2.1 - Diagrama de bloco do sistema de coincidência típico. 
Considerando-se uma fonte puntiforme de um emissor genérico com esquema de desintegração simples (Figura 2.2), em que a desintegração se dá pela emissão de uma partícula $\beta^{-}$seguida por um fóton gama, as taxas de contagem registradas em cada uma das vias de detecção são dadas por:

$$
\begin{aligned}
& \mathrm{N}_{\beta}=\mathrm{N}_{0} \varepsilon_{\beta} \\
& \mathrm{N}_{\gamma}=\mathrm{N}_{0} \varepsilon_{\gamma} \\
& \mathrm{N}_{\mathrm{c}}=\mathrm{N}_{0} \varepsilon_{\beta} \varepsilon_{\gamma}
\end{aligned}
$$

na qual

$\mathrm{N}_{0}$ atividade da fonte;

$\mathrm{N}_{\beta} \quad$ taxa de contagens na via beta;

$\mathrm{N}_{\gamma}$ taxa de contagens na via gama;

$\mathrm{N}_{\mathrm{c}}$ taxa de contagens de coincidências;

$\varepsilon_{\beta} \quad$ probabilidade de detecção da via beta e

$\varepsilon_{\gamma} \quad$ probabilidade de detecção da via gama.

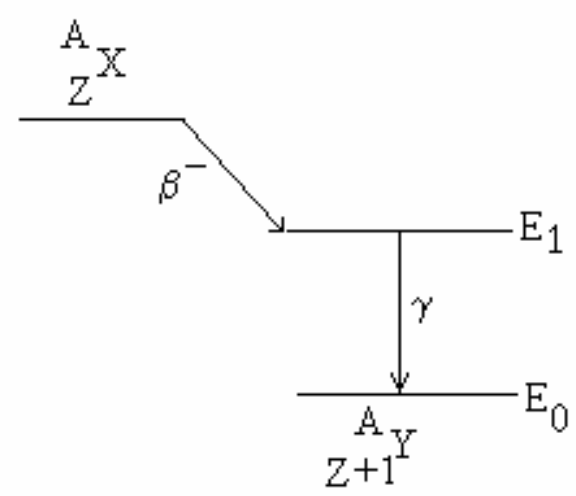

Figura 2.2 - Esquema de desintegração simples para um emissor $\beta-\gamma$ genérico.

Multiplicando-se a equação (2.1) por (2.2) e dividindo por (2.3), tem-se:

$$
\frac{\mathrm{N}_{\beta} \mathrm{N}_{\gamma}}{\mathrm{N}_{\mathrm{c}}}=\mathrm{N}_{0}
$$


Portanto, a atividade do radionuclídeo $\left(\mathrm{N}_{0}\right)$ é função apenas das taxas de contagens observadas, não dependendo do conhecimento de nenhum outro dado. Neste caso simples, as eficiências também podem ser determinadas substituindo a equação (2.4) nas equações (2.1) e (2.2) obtendo-se:

$$
\begin{gathered}
\varepsilon_{\beta}=\frac{\mathrm{N}_{\mathrm{c}}}{\mathrm{N}_{\gamma}} \\
\varepsilon_{\gamma}=\frac{\mathrm{N}_{\mathrm{c}}}{\mathrm{N}_{\beta}}
\end{gathered}
$$

\subsubsection{Técnica da Extrapolação da Eficiência}

Na prática, a desintegração apresentado no esquema anterior (Figura 2.2), corresponde a uma condição ideal que é raramente alcançada. Geralmente, os radionuclídeos apresentam esquemas de desintegração mais complexos, com vários ramos beta seguidos de transição gama ou da emissão de elétrons de conversão interna. Desse modo, uma série de correções devem ser aplicadas ${ }^{[1-7]}$, visto que não podem ser descartados outros parâmetros intrínsecos ao método de medida como sensibilidade de um dos detectores ao outro tipo de radiação, a qual ele não se destina, uso de fontes não puntiformes, tempos de resposta diferentes para cada detector etc.

Para um caso prático, em que o radionuclídeo decai por $\beta-\gamma$ (ou Captura Eletrônica $-\gamma$ ) e apresenta um esquema de desintegração complexo, com $n$ ramos beta, as equações (2.1), (2.2), (2.3) são alteradas e podem ser reescritas como:

$$
\begin{gathered}
\mathrm{N}_{\beta}=\mathrm{N}_{0} \sum_{\mathrm{i}=1}^{\mathrm{m}} \mathrm{a}_{\mathrm{i}}\left\{\varepsilon_{\beta_{\mathrm{i}}}+\left(1-\varepsilon_{\beta_{\mathrm{i}}}\right) \sum_{\mathrm{j}=1}^{\mathrm{n}} \mathrm{b}_{\mathrm{ij}} \frac{\left.\alpha_{\mathrm{ij}}\left[\varepsilon_{\mathrm{ec}_{\mathrm{ij}}}+\left(1-\varepsilon_{\mathrm{ec}_{\mathrm{ij}}}\right) \varepsilon(\mathrm{X}, \mathrm{A})_{\mathrm{ij}}\right)\right]+\varepsilon_{\gamma_{\mathrm{ij}}}}{1+\alpha_{\mathrm{ij}}}\right\} \\
\mathrm{N}_{\gamma}=\mathrm{N}_{0}\left(\sum_{\mathrm{i}=1}^{\mathrm{m}} \mathrm{a}_{\mathrm{i}} \sum_{\mathrm{j}=1}^{\mathrm{n}} \mathrm{b}_{\mathrm{ij}} \varepsilon_{\gamma_{\mathrm{ij}}} \frac{1}{1+\alpha_{\mathrm{ij}}}\right)
\end{gathered}
$$




$$
N_{c}=N_{0}\left\{\sum_{i=1}^{m} a_{i}\left[\varepsilon_{\beta_{i}} \sum_{j=1}^{n} b_{i j} \varepsilon_{\gamma_{i j}} \frac{1}{1+\alpha_{i j}}+\left(1-\varepsilon_{\beta_{i}}\right) \sum_{j=1}^{n} b_{i j} \varepsilon_{c_{i j}} \frac{1}{1+\alpha_{i j}}\right]\right\}
$$

na qual

$\mathrm{N}_{\beta}, \mathrm{N}_{\gamma}$ e $\mathrm{N}_{\mathrm{c}}$ são contagens beta, gama e coincidência, respectivamente;

$\mathrm{N}_{0}$ é a atividade;

$a_{i}$ e $b_{i j}$ são as intensidades por desintegração do i-ésimo ramo beta e intensidade relativa da j-ésima transição relacionada com o i-ésima ramo;

n é o número de transições que seguem o i-ésimo ramo beta;

$\mathrm{m}$ é o número de ramos beta;

$\varepsilon_{\beta_{\mathrm{i}}}$ é a eficiência beta associada ao i-ésimo ramo beta;

$\varepsilon_{\gamma_{\mathrm{ij}}}$, e $\varepsilon_{\beta \gamma_{\mathrm{ij}}}$ são eficiências de detecção gama e eficiência gama para detector beta, respectivamente, associada a ij-ésima transição;

$\varepsilon_{\mathrm{ec}_{\mathrm{ij}}}$ e $\varepsilon_{(\mathrm{X}, \mathrm{A})_{\mathrm{ij}}}$ são eficiências de detecção para elétron de conversão e elétron Auger ou eficiência de detecção para raio-X, respectivamente, associada a ij-ésima transição e,

$\varepsilon_{C_{i j}}$ e $\alpha_{i j}$ são a eficiência de detecção da coincidência gama-gama e o coeficiente de conversão interna da ij-ésima transição.

e a equação (2.4) passa a ser reescrita como:

$\frac{\mathrm{N}_{\beta} \mathrm{N}_{\gamma}}{\mathrm{N}_{\mathrm{c}}}=$

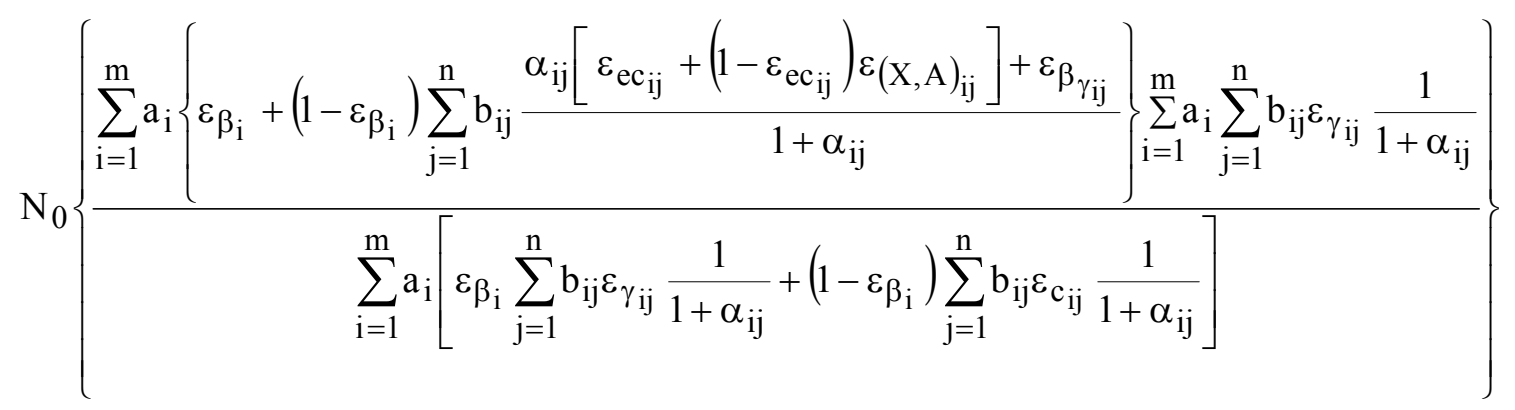

A medida da eficiência beta pode ser escrita como: 


$$
\frac{N_{c}}{N_{\gamma}}=\frac{\sum_{i=1}^{m} a_{i}\left[\varepsilon_{\beta_{i}} \sum_{j=1}^{n} b_{i j} \varepsilon_{\gamma_{i j}} \frac{1}{1+\alpha_{i j}}+\left(1-\varepsilon_{\beta_{i}}\right) \sum_{j=1}^{n} b_{i j} \varepsilon_{c_{i j}} \frac{1}{1+\alpha_{i j}}\right]}{\sum_{i=1}^{m} a_{i} \sum_{j=1}^{n} b_{i j} \varepsilon_{\gamma_{i j}} \frac{1}{1+\alpha_{i j}}}
$$

A equação (2.10) determina que para obtermos $\mathrm{N}_{0}$ é necessário conhecer os parâmetros do esquema de desintegração e as probabilidades de detecção. Para solucionar esse problema e manter a principal característica do método que consiste na sua independência do conhecimento das eficiências de detecção e dos parâmetros do esquema de desintegração, foi desenvolvida a Técnica da Extrapolação da Eficiência ${ }^{[3]}$.

Segundo esta técnica, a determinação da taxa de desintegração de um nuclídeo com esquema de desintegração complexo, sem o conhecimento prévio dos parâmetros do esquema de desintegração e das eficiências de detecção, só será possível se pudermos estabelecer uma relação funcional entre a contagem no detector beta, $\mathrm{N}_{\beta}$, e o parâmetro de eficiência $\frac{\mathrm{N}_{\mathrm{c}}}{\mathrm{N}_{\gamma}}$ tal que $\mathrm{N}_{\beta} \rightarrow \mathrm{N}_{0}$ quando $\frac{\mathrm{N}_{\mathrm{c}}}{\mathrm{N}_{\gamma}} \rightarrow 1$

Esta condição ocorre quando a eficiência dos vários ramos beta, $\varepsilon_{\beta_{\mathrm{r}}}$, puder ser representada como uma função de uma única eficiência $\varepsilon_{\beta_{\mathrm{s}}}$, isto é,

$$
\varepsilon_{\beta_{\mathrm{r}}}=\mathrm{f}_{\mathrm{r}}\left(\varepsilon_{\beta_{\mathrm{s}}}\right)
$$

e é imprescindível que esta relação se conserve para o caso em que $f_{r}$ tenda para 1 , quando uma das eficiências beta, $\varepsilon_{\beta_{\mathrm{s}}}$, tender para a unidade, ou seja,

$$
\mathrm{f}_{\mathrm{r}} \rightarrow 1 \text { quando } \varepsilon_{\beta_{\mathrm{s}}} \rightarrow 1 \text { para } \mathrm{r}=1,2, \cdots, \mathrm{n}
$$

além de que os valores das probabilidades de detecção $\varepsilon_{\gamma_{\mathrm{r}}}, \varepsilon_{\beta_{\gamma_{\mathrm{r}}}}, \varepsilon_{\mathrm{c}_{\mathrm{r}}}$ e $\varepsilon_{\mathrm{ec}_{\mathrm{r}}}$ permaneçam constantes no intervalo de variação $\frac{\mathrm{N}_{\mathrm{c}}}{\mathrm{N}_{\gamma}}$. 
Portanto, $\mathrm{N}_{\beta}$ resulta como uma função $\mathrm{F}$ do parâmetro de eficiência $\frac{\mathrm{N}_{\mathrm{c}}}{\mathrm{N}_{\gamma}}$, tal que:

$$
\mathrm{N}_{\beta}=\mathrm{N}_{0} \mathrm{~F}\left(\frac{\mathrm{N}_{\mathrm{c}}}{\mathrm{N}_{\gamma}}\right)
$$

em que $\mathrm{F} \rightarrow 1$ e $\quad \mathrm{N}_{\beta} \rightarrow \mathrm{N}_{0} \quad$ quando $\quad \frac{\mathrm{N}_{\mathrm{c}}}{\mathrm{N}_{\gamma}} \rightarrow 1$. A equação (2.12) é a fórmula de coincidência generalizada ${ }^{[1,3]}$.

A fórmula de coincidência generalizada pode ser posta em uma forma mais conveniente para sua solução gráfica ou analítica. Usando-se $\frac{\mathrm{N}_{\beta} \mathrm{N}_{\gamma}}{\mathrm{N}_{\mathrm{c}}}$ como variável dependente e $\frac{1-\frac{\mathrm{N}_{\mathrm{c}}}{\mathrm{N}_{\gamma}}}{\frac{\mathrm{N}_{\mathrm{c}}}{\mathrm{N}_{\gamma}}}$ como parâmetro variável, obtém-se uma função G do parâmetro de ineficiência, cuja variação é mais lenta do que a da função F referida na equação (2.12).

Assim a fórmula de coincidência generalizada pode ser escrita como:

$$
\frac{\mathrm{N}_{\beta} \mathrm{N}_{\gamma}}{\mathrm{N}_{\mathrm{c}}}=\mathrm{N}_{0} \mathrm{G}\left(\frac{1-\frac{\mathrm{N}_{\mathrm{c}}}{\mathrm{N}_{\gamma}}}{\frac{\mathrm{N}_{\mathrm{c}}}{\mathrm{N}_{\gamma}}}\right)
$$

na qual a função $\mathrm{G} \rightarrow 1$ e $\frac{\mathrm{N}_{\beta} \mathrm{N}_{\gamma}}{\mathrm{N}_{\mathrm{c}}} \rightarrow \mathrm{N}_{0}$ quando $\quad \frac{1-\frac{\mathrm{N}_{\mathrm{c}}}{\mathrm{N}_{\gamma}}}{\frac{\mathrm{N}_{\mathrm{c}}}{\mathrm{N}_{\gamma}}} \rightarrow 0$.

A variação do parâmetro de eficiência pode ser obtida pelo uso de absorvedores externos, ou auto-absorção na fonte, discriminação eletrônica ou qualquer outro método que possibilite a variação da eficiência beta. 
Determina-se a taxa de desintegração $\mathrm{N}_{0}$ por meio da extrapolação obtida por ajuste polinomial dos dados experimentais a partir do gráfico de $\frac{\mathrm{N}_{\beta} \mathrm{N}_{\gamma}}{\mathrm{N}_{\mathrm{c}}}$ versus $\frac{1-\frac{\mathrm{N}_{\mathrm{c}}}{\mathrm{N}_{\gamma}}}{\frac{\mathrm{N}_{\mathrm{c}}}{\mathrm{N}_{\gamma}}}$.

\subsubsection{Técnica do Traçador}

A padronização de um emissor beta puro geralmente é feita utilizando a técnica do traçador ${ }^{[47]}$. Nesse procedimento um emissor beta-gama conveniente é utilizado como traçador e misturado na solução do beta puro.

As equações gerais de coincidência aplicada para esta técnica podem ser escritas como:

$$
\begin{aligned}
& \mathrm{N}_{\beta}=\mathrm{N}_{0 \mathrm{P}} \varepsilon_{\beta \mathrm{P}}+ \\
& +\mathrm{N}_{0 \mathrm{~T}} \sum_{\mathrm{i}=1}^{\mathrm{m}} \mathrm{a}_{\mathrm{i}}\left\{\varepsilon_{\beta_{\mathrm{i}}}+\left(1-\varepsilon_{\beta_{\mathrm{i}}}\right) \sum_{\mathrm{j}=1}^{\mathrm{n}} \mathrm{b}_{\mathrm{ij}} \frac{\left.\alpha_{\mathrm{ij}}\left[\varepsilon_{\mathrm{ec}}+\left(1-\varepsilon_{\mathrm{ec}}\right) \varepsilon_{\mathrm{ij}}(\mathrm{X}, \mathrm{A})_{\mathrm{ij}}\right)\right]+\varepsilon_{\beta_{\gamma_{\mathrm{j}}}}}{1+\alpha_{\mathrm{ij}}}\right\}_{\mathrm{T}} \\
& \mathrm{N}_{\gamma \mathrm{T}}=\mathrm{N}_{0 \mathrm{~T}}\left(\sum_{\mathrm{i}=1}^{\mathrm{m}} \mathrm{a}_{\mathrm{i}} \sum_{\mathrm{j}=1}^{\mathrm{n}} \mathrm{b}_{\mathrm{ij}} \varepsilon_{\gamma_{\mathrm{ij}}} \frac{1}{1+\alpha_{\mathrm{ij}}}\right)_{\mathrm{T}} \\
& \mathrm{N}_{\mathrm{cT}}=\mathrm{N}_{0 \mathrm{~T}}\left\{\sum_{\mathrm{i}=1}^{\mathrm{m}}\left[\mathrm{a}_{\mathrm{i}}\left[\varepsilon_{\beta_{\mathrm{i}}} \sum_{\mathrm{j}=1}^{\mathrm{n}} \mathrm{b}_{\mathrm{ij}} \varepsilon_{\gamma_{\mathrm{ij}}} \frac{1}{1+\alpha_{\mathrm{ij}}}+\left(1-\varepsilon_{\beta_{\mathrm{i}}}\right) \sum_{\mathrm{j}=1}^{\mathrm{n}} \mathrm{b}_{\mathrm{ij}} \varepsilon_{\mathrm{c}_{\mathrm{ij}}} \frac{1}{1+\alpha_{\mathrm{ij}}}\right]\right\}_{\mathrm{T}}\right.
\end{aligned}
$$

assim 
$\frac{\mathrm{N}_{\beta} \mathrm{N}_{\gamma}}{\mathrm{N}_{\mathrm{c}}}=\mathrm{N}_{0 \mathrm{P}} \varepsilon_{\beta \mathrm{P}} \frac{\mathrm{N}_{\gamma}}{\mathrm{N}_{\mathrm{c}}}+$

$$
+N_{0 T}\left\{\frac{\sum_{i=1}^{m} a_{i}\left\{\varepsilon_{\beta_{i}}+\left(1-\varepsilon_{\beta_{i}}\right) \sum_{j=1}^{n} b_{i j} \frac{\alpha_{i j}\left[\varepsilon_{e c_{i j}}+\left(1-\varepsilon_{e c_{i j}}\right) \varepsilon_{(x, A)_{i j}}\right]+\varepsilon_{\beta_{\gamma_{i j}}}}{1+\alpha_{i j}}\right\} \sum_{i=1}^{m} a_{i} \sum_{j=1}^{n} b_{i j} \varepsilon_{\gamma_{i j}} \frac{1}{1+\alpha_{i j}}}{\sum_{i=1}^{m} a_{i}\left[\varepsilon_{\beta_{i}} \sum_{j=1}^{n} b_{i j} \varepsilon_{\gamma_{i j}} \frac{1}{1+\alpha_{i j}}+\left(1-\varepsilon_{\beta_{i}}\right) \sum_{j=1}^{n} b_{i j} \varepsilon_{c_{i j}} \frac{1}{1+\alpha_{i j}}\right]}\right\}
$$

na qual:

$P$ e $T$ são índices relacionados com emissores puro e beta-gama, respectivamente. Os demais parâmetros foram definidos no item 2.1.2.

Em casos especiais, para os quais o pico de absorção total de energia no canal gama pode ser selecionado para um único gama (com intensidade b por desintegração e coeficiente de conversão $\alpha$ ), as coincidências gama-gama são eliminadas $\left(\varepsilon_{\mathrm{c}_{\mathrm{ij}}}\right)$ e as equações 2.17 e 2.11 podem ser simplificadas:

$$
\begin{gathered}
\frac{\mathrm{N}_{\beta} \mathrm{N}_{\gamma}}{\mathrm{N}_{\mathrm{c}}}=\mathrm{N}_{0 \mathrm{P}} \varepsilon_{\beta \mathrm{P}} \frac{\mathrm{N}_{\gamma}}{\mathrm{N}_{\mathrm{c}}}+\mathrm{N}_{0 \mathrm{~T}}\left\{1+\mathrm{b} \frac{\left(1-\varepsilon_{\beta}\right)}{\varepsilon_{\beta}} \frac{\alpha\left[\varepsilon_{\mathrm{CE}}+\left(1-\varepsilon_{\mathrm{CE}}\right) \varepsilon(\mathrm{X}, \mathrm{A})\right]+\varepsilon_{\beta \gamma}}{1+\alpha}\right\}_{\mathrm{T}} \\
\frac{\mathrm{N}_{\mathrm{c}}}{\mathrm{N}_{\gamma}}=\varepsilon_{\beta \mathrm{T}}
\end{gathered}
$$

na qual

$$
\varepsilon_{\beta T}=\left\{\sum_{i=1}^{m} a_{i} \varepsilon_{\beta i}\right\}_{T}
$$




\subsection{Arranjo Experimental do Sistema de Coincidência $4 \pi(\mathrm{PC}) \beta-\gamma$}

O sistema de coincidência $4 \pi(\mathrm{PC}) \beta-\gamma$ utilizado no LMN do IPEN é constituído por um detector proporcional a gás fluente, com geometria $4 \pi$ para detecção da radiação $\beta$ $\left(\beta^{-}\right.$e $\left.\beta^{+}\right)$, elétrons de conversão, raios-X e elétrons Auger, acoplado a dois cristais cintiladores de $\mathrm{NaI}(\mathrm{Tl})$, para detecção da radiação gama. A figura 2.3 mostra o sistema de coincidência do LMN. Os eventos coincidentes são registrados quando detectados simultaneamente nos dois detectores $(4 \pi(\mathrm{PC})$ e $\mathrm{NaI}(\mathrm{Tl}))$.

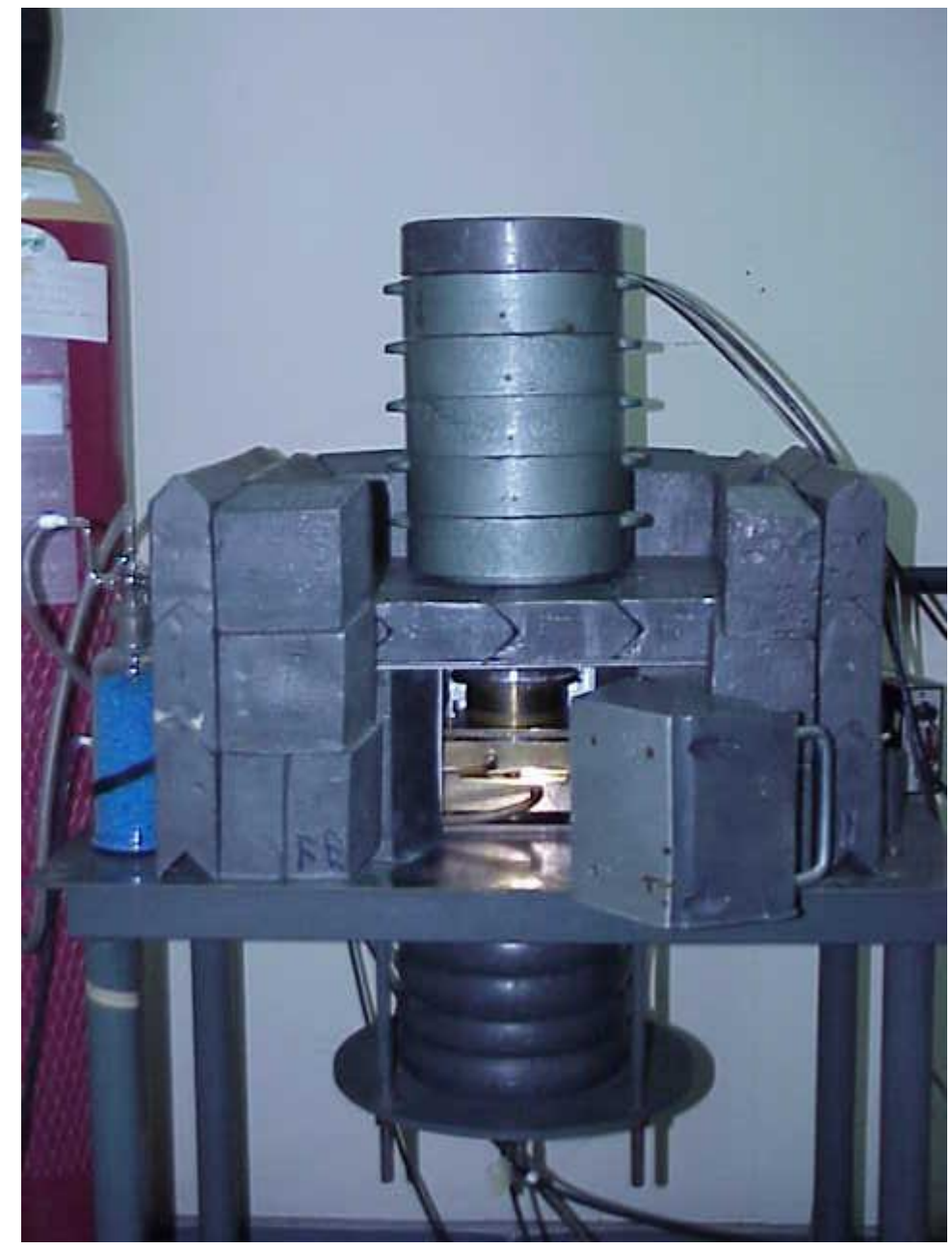

Figura 2.3 - Sistema de coincidência $4 \pi(\mathrm{PC})-\mathrm{NaI}(\mathrm{Tl})$ do $\mathrm{LMN}$. 


\subsubsection{Detector Proporcional com Geometria $4 \pi$}

O contador proporcional apresenta baixo ruído, baixa sensibilidade para radiação $\gamma(<1 \%)$, tempo de recuperação de $\approx 100 \mathrm{~ns}$, e alta eficiência na detecção de partículas $\beta$, entre $90 \%$ a $100 \%$.

O detector proporcional do LMN é composto por duas partes simétricas de latão, entre as quais é encaixada uma placa deslizante com dois orifícios para o posicionamento das fontes no interior do detector, sem a necessidade de abrí-lo. A vedação é garantida por um sistema de anéis de borracha dispostos em cada uma das metades. Seu volume interno é delimitado por definidores de latão, que o tornam aproximadamente cilíndrico quando as duas partes do conjunto são justapostas, tendo $3,0 \mathrm{~cm}$ de diâmetro e comprimento de $7,5 \mathrm{~cm}$, como mostra as figuras 2.4 (a) e (b).
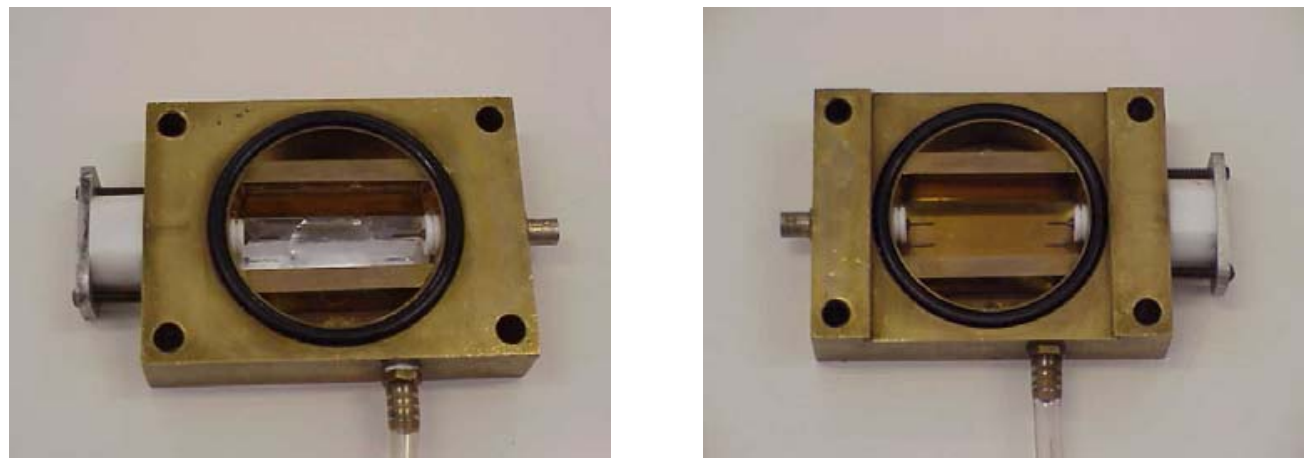

Figura 2.4 - Contador proporcional do LMN, com suas duas metades separadas para ilustrar o interior do detector. $\mathrm{Na}$ ilustração (b) percebe-se a fenda para encaixar a placa deslizante para o posicionamento da fonte no seu interior.

Em cada metade do detector estende-se, paralelamente ao eixo do cilindro, um anodo, formado por um fio de aço inoxidável, com diâmetro de $25 \mu \mathrm{m}$, muito bem esticado, preso por isoladores de teflon em um dos lados e ligados a conectores de alta tensão do outro lado. A figura 2.5 mostra o esquema do contador proporcional, em corte transversal e vista frontal. 


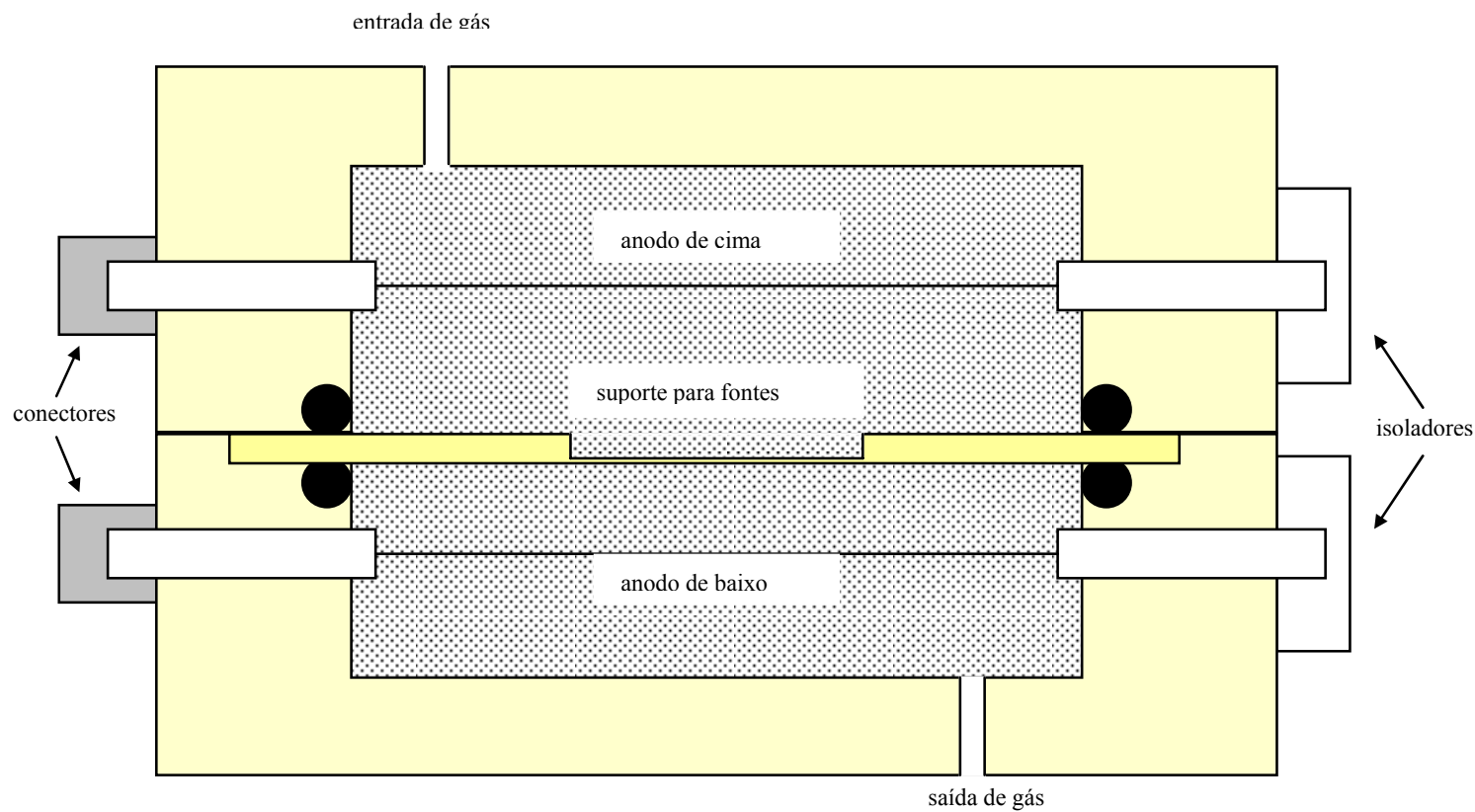

Figura 2.5 - Esquema do contador proporcional, em corte transversal e vista frontal.

O interior do contador é preenchido com gás de alta pureza constituído de $90 \%$ de argônio e 10\% de metano (mistura $\mathrm{P} 10$ ), operado à pressão de $0,1 \mathrm{MPa}$ (pressão atmosférica). O contador proporcional do LMN também pode ser pressurizado, acoplandose um sistema de alta pressão.

As partículas emitidas pela fonte ionizam as moléculas do gás. Os elétrons livres assim formados são acelerados pelo campo elétrico no interior do detector em direção ao anodo, ganhando energia no percurso e ionizando outras moléculas do gás, originando uma avalanche, conhecida como avalanche Towsend ${ }^{[42]}$. Essa avalanche termina quando todos os elétrons livres são coletados no anodo, sendo a corrente formada proporcional ao número de elétrons, que por sua vez, é proporcional à energia depositada pela partícula, uma vez que o detector é operado na região proporcional, com um fator de multiplicação de aproximadamente $10^{4}$.

\subsubsection{Cristal Cintilador de NaI(TI)}

As janelas de alumínio (espessura de $0,3 \mathrm{~mm}$ ) de dois cristais cintiladores de $\mathrm{NaI}$ (Iodeto de Sódio) ativado com Tálio (Tl), com dimensões de $76 \mathrm{~mm}$ x $76 \mathrm{~mm}$, estão posicionados em contato direto com as faces superior e inferior do contador proporcional. 
Os fótons emitidos pela fonte radioativa incidem sobre a superfície do cristal produzindo ionização que podem ser detectadas pela emissão de cintilações através das quais as moléculas do cristal retornam ao estado fundamental. A luz visível assim gerada incide em uma célula fotomultiplicadora, que a converterá em elétrons que, por sua vez, serão multiplicados. Esta corrente elétrica produz um pulso cuja altura é proporcional à energia perdida pela radiação e/ou partículas no interior do cristal.

\subsubsection{Sistema Eletrônico Associado}

A cada um dos detectores são acoplados equipamentos eletrônicos que permitem os ajustes necessários para a medida e aquisição de dados. $\mathrm{O}$ sistema eletrônico básico utilizado é apresentado na figura $2.6^{[24]}$.

Este sistema é composto por duas vias distintas, $\beta$ e $\gamma$, cada uma destinada a um tipo de detector.

- Via $\beta$ - Detector proporcional $4 \pi$ : Os pulsos provenientes dos dois anodos distintos são somados gerando um único sinal que passa por um estagio de amplificação. A medida nesta via é feita selecionando-se a região do espectro beta acima no ruído eletrônico. Para isso faz-se a discriminação por meio de um analisador monocanal com atrasador (Single Channel Analyser-Timing, ou simplesmente SCA-Timing). Após esta discriminação, os pulsos são enviados a um módulo de porta e atraso (Gate and Delay Generator) onde é fixado o atraso para a separação dos pulsos no espectro de aquisição de dados, sendo posteriormente enviados para um conversor de tempo em altura de pulso (módulo TAC) para o registro dos eventos.

- Via $\gamma$ - Detector cintilador de NaI(Tl): Os pulsos provenientes dos dois detectores distintos passam por amplificadores e são depois somados gerando um único sinal. A medida nesta via é feita selecionando-se a região do espectro gama referente a um ou mais picos de absorção total; para isso faz-se a discriminação destes por meio de um analisador monocanal com atrasador (Single Channel Analyser-Timing, ou 
simplesmente SCA-Timing) que permite selecionar a faixa de energia de interesse janela. Após esta seleção, os pulsos são tratados de modo semelhante à via beta.

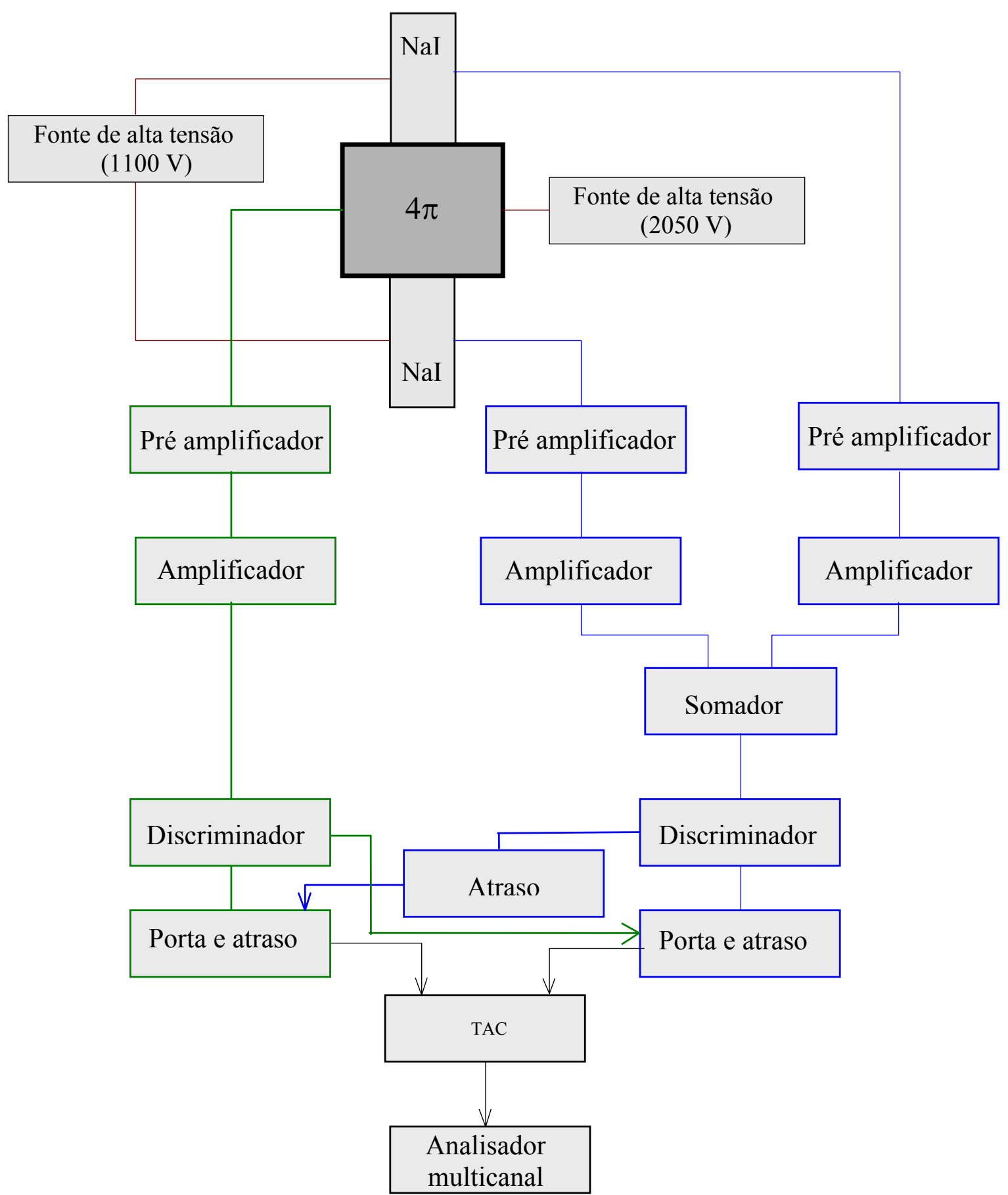

Figura 2.6 - Diagrama do arranjo eletrônico convencional utilizado no sistema de coincidências $4 \pi \beta-\gamma$. 
O método utilizado para o registro das contagens foi desenvolvido no LMN do IPEN e utiliza um módulo TAC, acoplado a um multicanal, onde é feito o registro dos espectros de aquisição de dados ${ }^{[48]}$.

Para isso, o módulo TAC recebe os pulsos provenientes das vias, $\gamma$ e $\beta$, após passagem por módulos de porta e atraso. Uma vez no TAC, um dos pulsos dará o início start e o outro o término stop, havendo o registro das contagens do seguinte modo: uma contagem $\beta$ quando ambos os pulsos forem provenientes do detector proporcional; uma contagem $\gamma$ quando ambos os pulsos forem provenientes dos cintiladores e uma contagem de coincidência quando for detectado um pulso de cada uma das vias.

\subsubsection{Preparação de Fontes Radioativas}

As fonte radioativas utilizadas no sistema de coincidências, devem ser preparadas em substratos de plásticos (filmes) finos o suficiente para evitar que haja a auto-absorção de elétrons ou raio-X de baixa energia.

Os substratos utilizados na confecção das fontes para medida no sistema de coincidência $4 \pi \beta-\gamma$, são filmes finos de Collodion (nitrato de celulose, com espessura de $\sim 10 \mu \mathrm{g} \cdot \mathrm{cm}^{-2}$ ) aderidos a arandelas de aço inoxidável com $0,1 \mathrm{~mm}$ de espessura, diâmetro interno de $2 \mathrm{~cm}$ e externo de $4 \mathrm{~cm}$. Como o sistema de coincidência utilizado tem como pré-requisito o uso de substratos condutores, o conjunto é metalizado com Au (densidade superficial de $\sim 10 \mu \mathrm{g} \cdot \mathrm{cm}^{-2}$ ), tornando-o condutor para que, uma vez colocado no interior do detector proporcional, não interfira no seu campo elétrico.

As fontes são preparadas depositando-se sobre o centro do substrato alíquotas conhecidas da solução radioativa. Durante o processo de secagem, pode ocorrer a cristalização de resíduos não uniformes. Para evitar a formação destes cristais e garantir a homogeneidade do conjunto, adiciona-se uma gota de Cyastat S.N. (concentração de 1\%), um agente que rompe a tensão superficial do líquido. Faz-se a secagem com jato aquecido de nitrogênio $\left(45{ }^{0} \mathrm{C}\right)$, reduzindo a formação de cristais maiores, produzidos com a secagem rápida. Na figura 2.7 é mostrado um esquema das fontes. 
Collodion metalizado com $\mathrm{Au}$

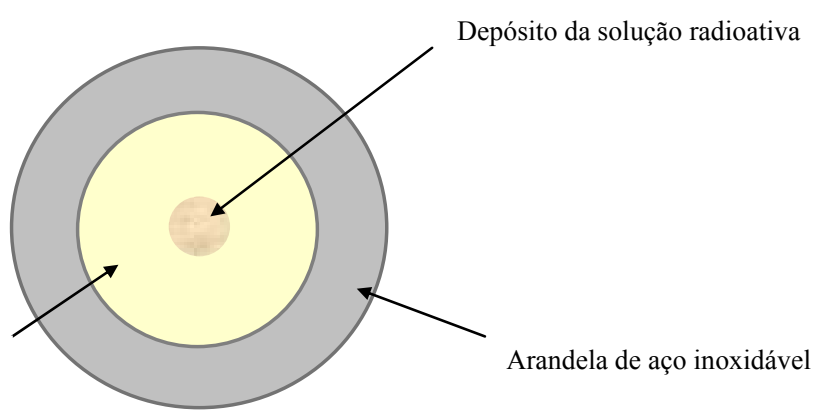

Figura 2.7 - Esquema das fontes para as medidas no sistema de coincidências $4 \pi \beta-\gamma$

A massa das fontes é determinada pelo método do picnômetro, que consiste na determinação da massa da alíquota pela diferença obtida entre pesagens consecutivas do picnômetro que contém a solução radioativa, antes e depois da deposição da alíquota no substrato. A pesagem do picnômetro é feita em balança analítica da marca Mettler, modelo M5SA, ou Sartorius MC 21S, com incerteza da ordem de $15 \mu \mathrm{g}$, sendo a massa da fonte aproximadamente da ordem de 1,7 mg.

\subsection{Simulação Teórica do Sistema de Coincidência $4 \pi(\mathrm{PC}) \beta-\gamma$}

Foi efetuada uma modelagem simplificada do arranjo experimental do sistema de coincidência $4 \pi(\mathrm{PC}) \beta-\gamma$ vista no item 2.2 para a simulação por meio da técnica de Monte Carlo da extrapolação da eficiência na medida da atividade, tomando-se por base informações da geometria do sistema de coincidência do LMN. Na figura 2.8 é apresentado esse modelo simplificado que é constituído por um detector proporcional e por um detector de $\mathrm{NaI}(\mathrm{Tl})$.

Este modelo é o que foi utilizado na geração das tabelas finais de eficiências no sistema de coincidências utilizadas na simulação da técnica da extrapolação da eficiência para determinar a atividade da fonte radioativa.

Os resultados obtidos utilizando este modelo estão de acordo com os resultados experimentais para a maioria dos casos estudados, mostrando que este modelo é uma aproximação razoável para o arranjo experimental. 


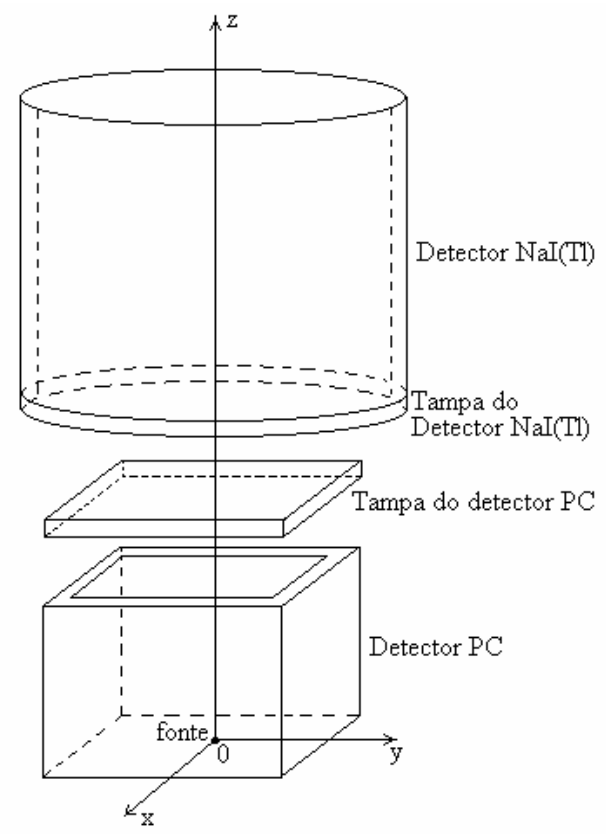

Figura 2.8 - Esquema simplificado do arranjo experimental do LMN utilizado na técnica de Monte Carlo.

\subsection{Padronização de Radionuclídeos por Meio de Sistemas de Coincidência}

Aplicou-se a metodologia desenvolvida no presente trabalho para a padronização de radionuclídeos que se desintegram por emissão de $\beta^{-}$e pelo processo de Captura Eletrônica. Os radionuclídeos empregados foram ${ }^{134} \mathrm{Cs}$ e ${ }^{72} \mathrm{Ga}$ para o primeiro caso, e ${ }^{133}$ Ba para o segundo.

Foi aplicada a metodologia também para o caso do ${ }^{35} \mathrm{~S}$ que é um emissor beta puro, por meio do Método do Traçador e utilizando-se ${ }^{60} \mathrm{Co}$ como radionuclídeo traçador.

\subsubsection{Padronização do ${ }^{134} \mathrm{Cs}$}

O radionuclídeo ${ }^{134} \mathrm{Cs}$ apresenta esquema de desintegração complexo e se desintegra com meia-vida de 2,066 anos e energia de desintegração total de 2058,7 keV. Vários ramos beta alimentam os níveis excitados do ${ }^{134} \mathrm{Ba}$ que, por sua vez, decaem originando várias transições gama ou por emissão de elétrons de conversão interna. O ramo beta mais intenso é o de $657,8 \mathrm{keV}$ e as transições gama mais intensas são de 604,69 keV e $795,84 \mathrm{keV}$. O ramo de Captura Eletrônica que alimenta o estado excitado de ${ }^{134} \mathrm{Xe}$ 
apresenta intensidade muito baixa $(0,0003 \%)$ e foi desprezado. O esquema de desintegração do ${ }^{134} \mathrm{Cs}$ é apresentado na figura $2.9^{[44]}$.

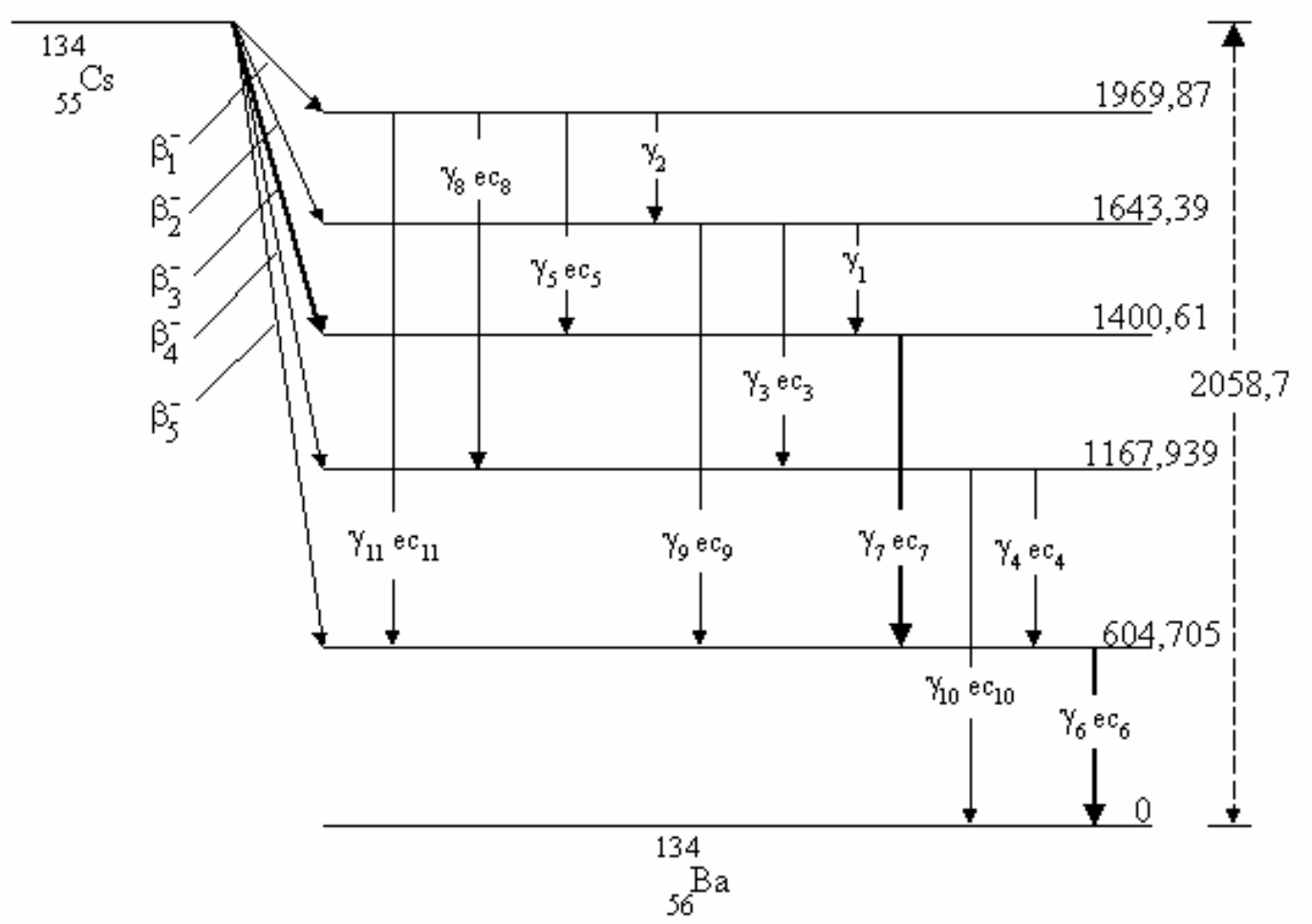

Figura 2.9 - Esquema de desintegração do ${ }^{134} \mathrm{Cs}$ (omitido ramo captura eletrônica)

Na tabela 2.1 são apresentadas as energias e probabilidades de emissão dos vários tipos de radiações emitidas na desintegração do ${ }^{134} \mathrm{Cs}^{[44]}$.

A padronização do ${ }^{134} \mathrm{Cs}$ foi realizada por meio de um sistema de coincidência convencional $4 \pi(\mathrm{PC}) \beta-\gamma^{[49]}$, constituído de um contador proporcional $4 \pi$ preenchido com uma mistura de gás $\mathrm{P}-10$ a pressão de $0,1 \mathrm{MPa}$, acoplado a um par de cristal de $\mathrm{NaI}(\mathrm{Tl})$ de $76 \mathrm{~mm}$ x $76 \mathrm{~mm}$. Uma descrição detalhada deste sistema pode ser obtida na referência 7 . Todos os pulsos acima da energia de corte do ruído $(0,7 \mathrm{keV})$ foram medidos no canal beta. Foram selecionadas duas janelas para os raios gama, abrangendo os picos de absorção total de $600 \mathrm{keV}$ e $800 \mathrm{keV}$, respectivamente. 
Tabela 2.1 - Tipos de radiação, energia e probabilidade de emissão para o processo de desintegração por $\beta^{-}$do ${ }^{134} \mathrm{Cs}$.

\begin{tabular}{|c|c|c|c|c|}
\hline $\begin{array}{l}\text { Tipo de } \\
\text { radiação }\end{array}$ & $\begin{array}{c}\text { Energia } \\
(\mathrm{keV})\end{array}$ & $\begin{array}{c}\text { Probabilidade de } \\
\text { emissão por } \\
\text { desintegração } \\
(\%)\end{array}$ & \multicolumn{2}{|c|}{$\begin{array}{l}\text { Coeficiente de } \\
\text { conversão interna }\end{array}$} \\
\hline \multicolumn{5}{|l|}{ Transições $\beta^{-}$} \\
\hline$\beta_{1}^{-}$ & $88,5 \pm 0,4$ & $27,2 \pm 0,2$ & & \\
\hline$\beta_{2}^{-}$ & $415,1 \pm 0,4$ & $2,50 \pm 0,05$ & & \\
\hline$\beta_{3}^{-}$ & $657,8 \pm 0,4$ & $70,2 \pm 0,4$ & & \\
\hline$\beta_{4}^{-}$ & $890,5 \pm 0,4$ & $0,04 \pm 0,03$ & & \\
\hline$\beta_{5}^{-}$ & $1453,7 \pm 0,4$ & $0,008 \pm 0,006$ & & \\
\hline \multicolumn{3}{|l|}{ Transições $\gamma$} & $\alpha_{K}\left(10^{-3}\right)$ & $\alpha_{t}\left(10^{-3}\right)$ \\
\hline$\gamma_{1}$ & $242,8 \pm 0,1$ & $0,021 \pm 0,001$ & & \\
\hline$\gamma_{2}$ & $326,5 \pm 0,1$ & $0,014 \pm 0,001$ & & \\
\hline$\gamma_{3}$ & $475,34 \pm 0,02$ & $1,52 \pm 0,02$ & $9,5 \pm 0,2$ & $11,4 \pm 0,3$ \\
\hline$\gamma_{4}$ & $563,23 \pm 0,02$ & $8,44 \pm 0,03$ & $6,1 \pm 0,4$ & $7,3 \pm 0,5$ \\
\hline$\gamma_{5}$ & $569,32 \pm 0,02$ & $15,44 \pm 0,05$ & $8,2 \pm 0,5$ & $9,6 \pm 0,6$ \\
\hline$\gamma_{6}$ & $604,69 \pm 0,02$ & $98,21 \pm 0,04$ & $4,94 \pm 0,13$ & $5,9 \pm 0,3$ \\
\hline$\gamma_{7}$ & $795,84 \pm 0,01$ & $85,78 \pm 0,03$ & $2,58 \pm 0,07$ & $3,0 \pm 0,1$ \\
\hline$\gamma_{8}$ & $801,93 \pm 0,02$ & $8,73 \pm 0,02$ & $2,54 \pm 0,08$ & $3,0 \pm 0,1$ \\
\hline$\gamma_{9}$ & $1038,555 \pm 0,020$ & $0,993 \pm 0,004$ & $1,57 \pm 0,15$ & $1,8 \pm 0,1$ \\
\hline$\gamma_{10}$ & $1167,92 \pm 0,02$ & $1,794 \pm 0,008$ & $1,12 \pm 0,05$ & $1,31 \pm 0,07$ \\
\hline$\gamma_{11}$ & $1365,16 \pm 0,02$ & $3,018 \pm 0,013$ & $0,82 \pm 0,03$ & $0,96 \pm 0,04$ \\
\hline
\end{tabular}

\subsubsection{Padronização do ${ }^{72} \mathrm{Ga}$}

$\mathrm{O}^{72} \mathrm{Ga}$ decai com meia-vida de $14,10 \mathrm{~h}^{[50]}$ por emissão $\beta^{-}$alimentando o estado excitado de ${ }^{72} \mathrm{Ge}$ e possui energia de desintegração total igual a 4001,1 keV. Seu esquema de desintegração é apresentado na figura 2.10 e $2.11^{[51]}$. A padronização do ${ }^{72}$ Ga no 
sistema de coincidência $4 \pi \beta-\gamma$ é de interesse, em razão da presença de gamas na região de alta energia (em torno de $2000 \mathrm{keV}$ ) onde há poucos pontos de calibração. Para a calibração de espectrômetros gama nesta região de energia, geralmente é utilizado o ${ }^{24} \mathrm{Na}$, que apresenta duas transições gama intensas: $1369 \mathrm{keV}$ e $2754 \mathrm{keV}$. O ${ }^{72} \mathrm{Ga}$ pode ser utilizado como um padrão auxiliar por apresentar transições gama intensas de $834 \mathrm{keV}$, $2200 \mathrm{keV}$ e $2500 \mathrm{keV}$, preenchendo o intervalo entre $1369 \mathrm{keV}$ e $2754 \mathrm{keV}$.

Experimentalmente foram irradiadas duas amostras e foram preparadas duas fontes radioativas a partir de cada amostra. As medidas foram efetuadas selecionando-se duas janelas no canal gama que foram $(680+834) \mathrm{keV}$ (janela 1) e $(2202+2508) \mathrm{keV}$ $(\text { janela } 2)^{[20,24]}$. 


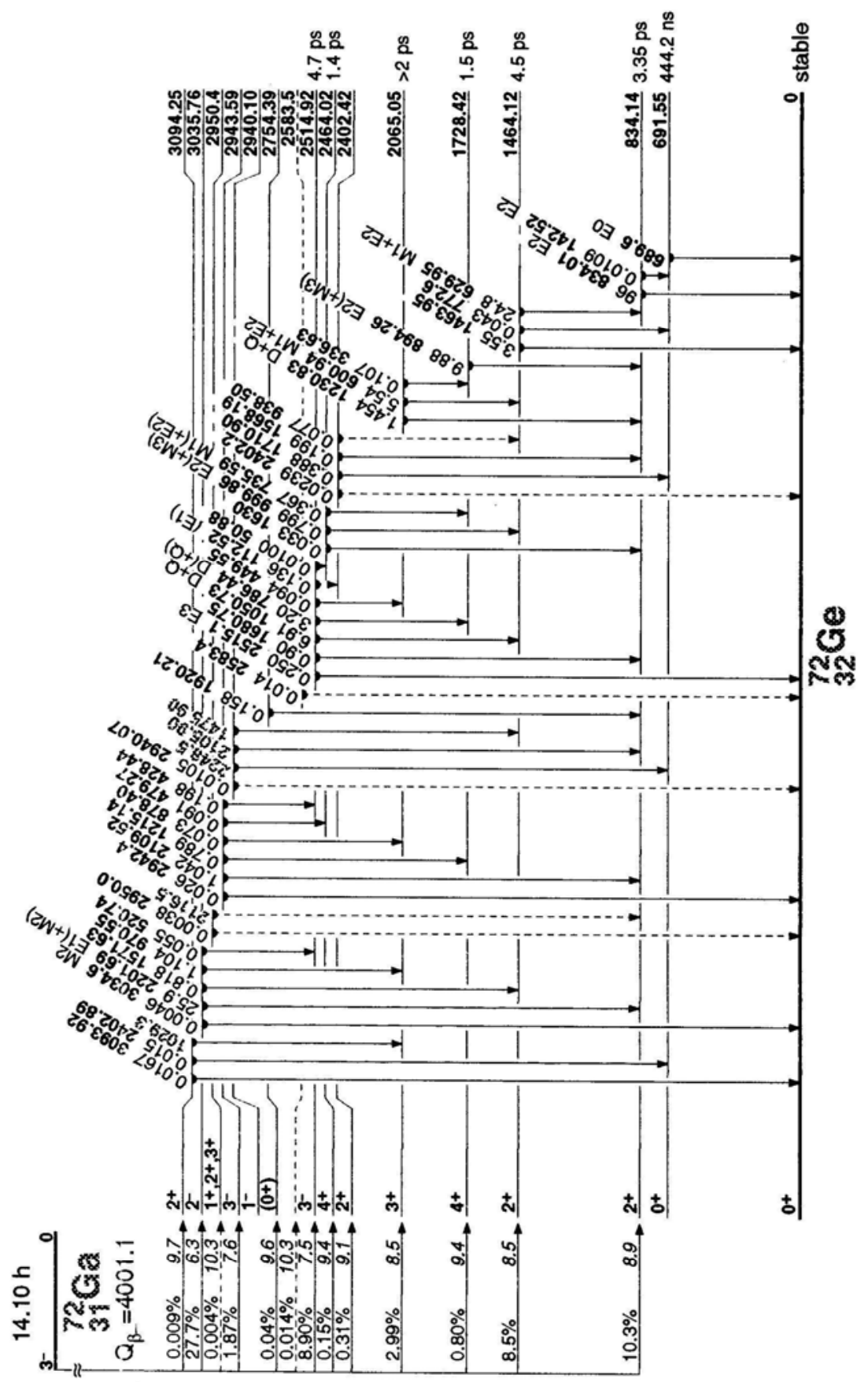

Figura 2.10 - Esquema de desintegração para o ${ }^{72} \mathrm{Ga}$, parte 1. 


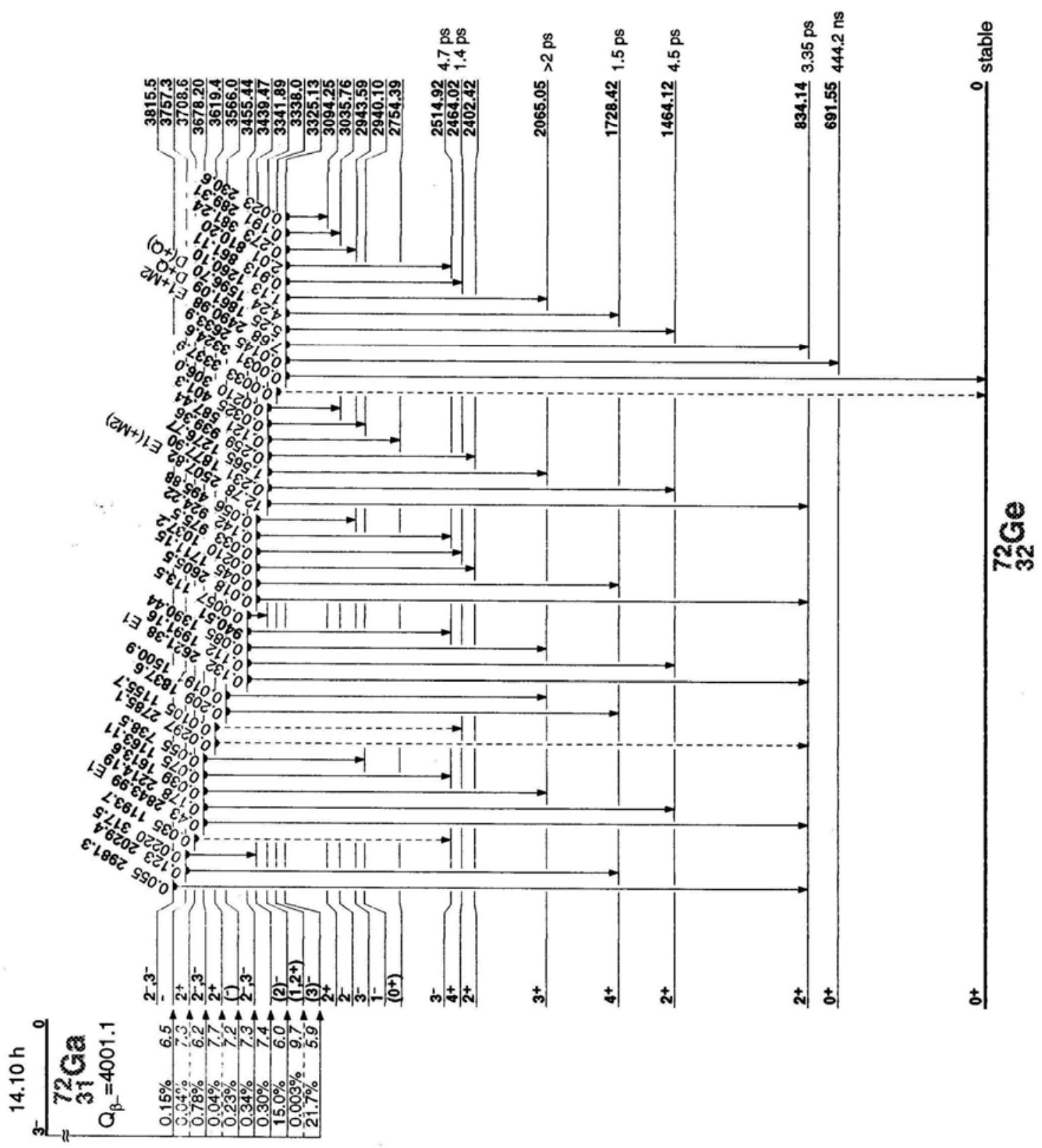

Figura 2.11- Esquema de desintegração do ${ }^{72} \mathrm{Ga}$, parte 2 .

Na tabela 2.2 estão as principais linhas de energia gama emitidas com suas respectivas probabilidades de emissão. 
Tabela 2.2 - Principais linhas de energias gama de desintegração do ${ }^{72} \mathrm{Ga}^{[51]}$.

\begin{tabular}{cc}
\hline Energia $(\mathrm{keV})$ & $\begin{array}{c}\text { Probabilidade de emissão } \\
\text { por desintegração } \%)\end{array}$ \\
\hline $600,95(3)$ & $5,53(11)$ \\
$629,96(4)$ & $24,76(48)$ \\
$786,44(8)$ & $3,20(6)$ \\
$810,20(9)$ & $2,008(38)$ \\
$834,03(3)$ & $95,63(7)$ \\
$861,11(5)$ & $0,913(20)$ \\
$894,25(10)$ & $9,87(16)$ \\
$999,86(6)$ & $0,798(14)$ \\
$1050,69(5)$ & $6,91(11)$ \\
$1230,86(7)$ & $1,453(19)$ \\
$1260,10(7)$ & $1,128(29)$ \\
$1276,76(7)$ & $1,564(16)$ \\
$1464,00(7)$ & $3,547(57)$ \\
$1596,68(8)$ & $4,236(86)$ \\
$1861,09(6)$ & $5,250(77)$ \\
$2201,66(7)$ & $25,91(47)$ \\
$2490,98(7)$ & $7,67(23)$ \\
$2507,79(7)$ & $12,77(23)$ \\
\hline
\end{tabular}

\subsubsection{Padronização do ${ }^{133} \mathrm{Ba}$}

O sistema de coincidência $4 \pi(\mathrm{PC}) \beta-\gamma$ também pode ser utilizado para a padronização de radionuclídeos que decaem por Captura Eletrônica e apresentam esquema de desintegração complexo. Para a verificação da presente metodologia, foi escolhido o ${ }^{133} \mathrm{Ba}$ porque foi alvo de uma comparação internacional patrocinada pelo BIPM (Bureau International dès Poids et Mèsures) ${ }^{[52]}$. O ${ }^{133} \mathrm{Ba}$ decai com meia vida de 10,6 anos, sendo a energia de desintegração de 520,4 keV. Apresenta dois ramos para captura eletrônica tendo 
o mais intenso energia de $83,4 \mathrm{keV}$ e duas transições gama mais intensas com energia de $80,998 \mathrm{keV}$ e $356,018 \mathrm{keV}$. Seu esquema de desintegração é apresentado na figura $2.12^{[44]}$.

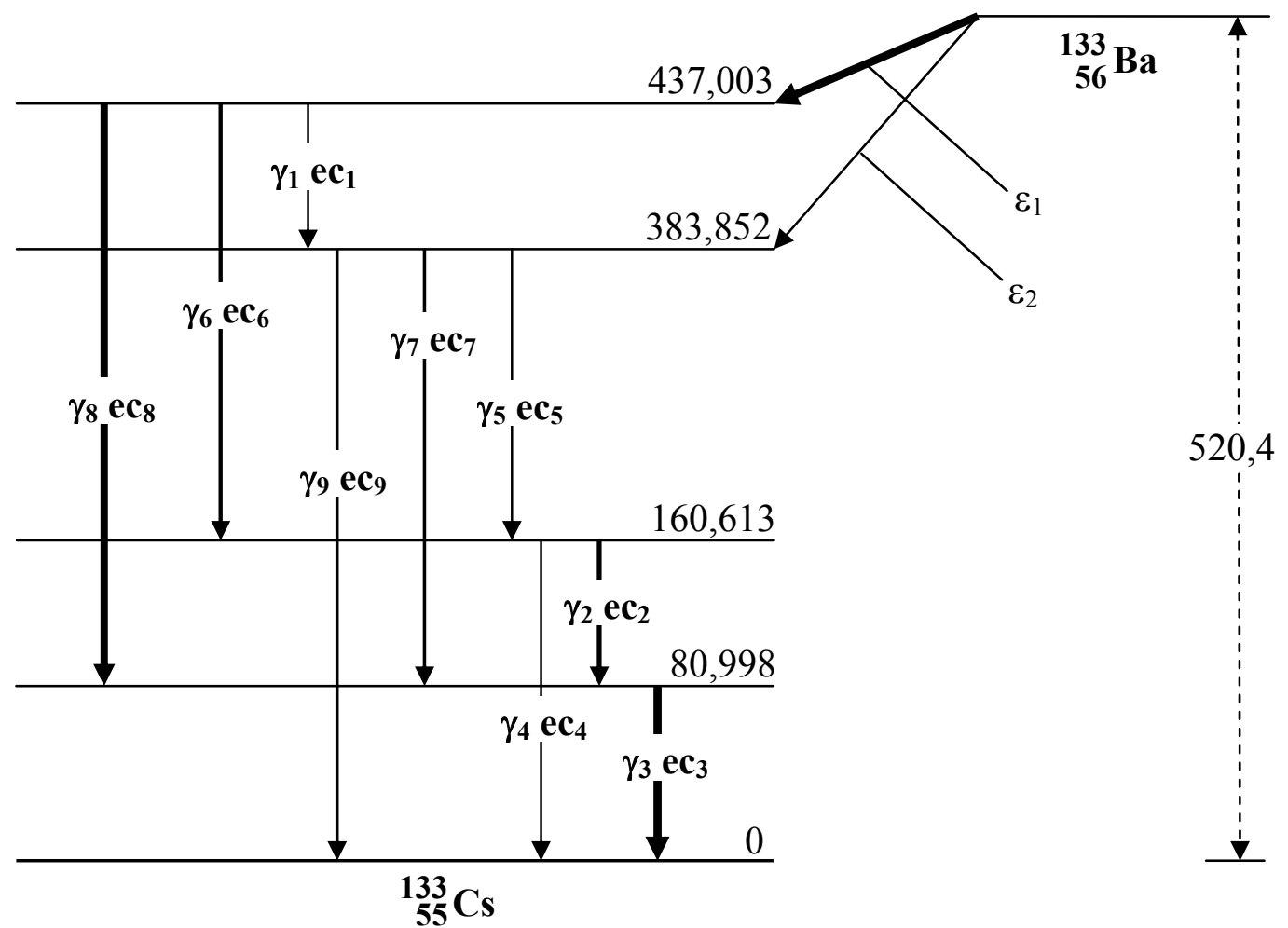

Figura 2.12 - Esquema de desintegração do ${ }^{133} \mathrm{Ba}$

Na tabela 2.3 são apresentadas as energias e intensidades dos vários tipos de radiações emitidas na desintegração do ${ }^{133} \mathrm{Ba}^{[44]}$.

Para a determinação experimental da atividade, o corte no nível de discriminação para o ruído no detector proporcional foi de $0,91 \mathrm{keV}$. A janela gama foi ajustada para abranger o intervalo de energias entre $222 \mathrm{keV}$ e $420 \mathrm{keV}$, aproximadamente. 
Tabela 2.3 - Tipos de radiação, energia e probabilidade de emissão para o esquema de desintegração por Captura Eletrônica do ${ }^{133} \mathrm{Ba}$.

\begin{tabular}{|c|c|c|c|c|}
\hline $\begin{array}{l}\text { Tipo de } \\
\text { radiação }\end{array}$ & $\begin{array}{c}\text { Energia } \\
(\mathrm{keV})\end{array}$ & $\begin{array}{c}\text { Probabilidade } \\
\text { de emissão por } \\
\text { desintegração } \\
(\%)\end{array}$ & & \\
\hline Captura Eletrônica & & & $\mathrm{P}_{\mathrm{K}}$ & $\mathrm{P}_{\mathrm{L}}$ \\
\hline$\varepsilon_{1}$ & $83,4 \pm 3,0$ & $85,9 \pm 0,6$ & $0,689 \pm 0,008$ & $0,247 \pm 0,007$ \\
\hline$\varepsilon_{2}$ & $136,6 \pm 3,0$ & $14,1 \pm 0,6$ & $0,775 \pm 0,006$ & $0,179 \pm 0,005$ \\
\hline Transição $\gamma$ & & & $\alpha_{K}$ & $\alpha_{t}$ \\
\hline$\gamma_{1}$ & $53,161 \pm 0,001$ & $14,65 \pm 0,35$ & $4,85 \pm 0,05$ & $5,66 \pm 0,06$ \\
\hline$\gamma_{2}$ & $79,623 \pm 0,010$ & $7,6 \pm 0,3$ & $1,4 \pm 0,1$ & $1,7 \pm 0,2$ \\
\hline$\gamma_{3}$ & $80,998 \pm 0,005$ & $90,10 \pm 0,20$ & $1,36 \pm 0,04$ & $1,64 \pm 0,05$ \\
\hline$\gamma_{4}$ & $160,613 \pm 0,008$ & $0,80 \pm 0,02$ & $0,205 \pm 0,007$ & $0,253 \pm 0,010$ \\
\hline$\gamma_{5}$ & $223,234 \pm 0,012$ & $0,49 \pm 0,02$ & $0,085 \pm 0,010$ & $0,090 \pm 0,010$ \\
\hline$\gamma_{6}$ & $276,398 \pm 0,002$ & $7,59 \pm 0,06$ & $0,048 \pm 0,002$ & $0,058 \pm 0,003$ \\
\hline$\gamma_{7}$ & $302,863 \pm 0,001$ & $19,1 \pm 0,2$ & $0,037 \pm 0,002$ & $0,043 \pm 0,003$ \\
\hline$\gamma_{8}$ & $356,018 \pm 0,010$ & $63,6 \pm 0,4$ & $\begin{array}{c}0,0211 \pm \\
0,0006\end{array}$ & $0,0256 \pm 0,0007$ \\
\hline$\gamma_{9}$ & $383,852 \pm 0,009$ & $9,1 \pm 0,1$ & $0,017 \pm 0,001$ & $0,021 \pm 0,001$ \\
\hline
\end{tabular}

Constantes atômicas
$\omega_{K}$
$0,895 \pm 0,012$
$\bar{\omega}_{L}$
$0,10 \pm 0,03$

Elétron Auger

$\begin{array}{lcc}\text { KLL } & 24,41-25,80 & 100 \\ \text { KLX } & 29,00-30,96 & 46,8 \\ \text { KXY } & 33,51-35,95 & 6,4\end{array}$

$\mathrm{L}$

$2,5-5,6$ 


\subsubsection{Padronização do ${ }^{35} \mathrm{~S}$}

Este caso corresponde à medida de um emissor beta puro, aplicando-se o Método do Traçador. O radionuclídeo escolhido para esta padronização foi o ${ }^{35} \mathrm{~S}$ que se desintegra por desintegração $\beta^{-}$para o estado fundamental do núcleo estável do ${ }^{35} \mathrm{Cl}$ (a figura 2.13 mostra o esquema de desintegração ${ }^{[44]}$ ). Foi utilizado como traçador o ${ }^{60}$ Co que se desintegra por emissão $\beta^{-}$populando os níveis excitados do ${ }^{60} \mathrm{Ni}$ (figura 2.14 mostra o esquema de desintegração) misturado na solução com o beta puro.

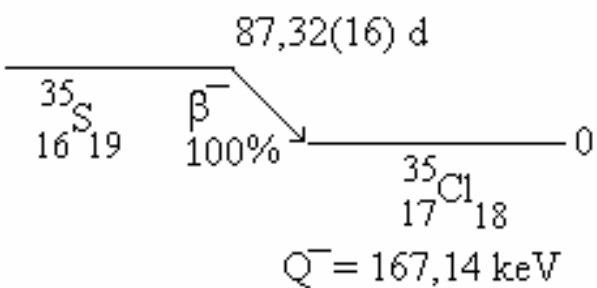

Figura 2.13 - Esquema de desintegração do ${ }^{35} \mathrm{~S}$.

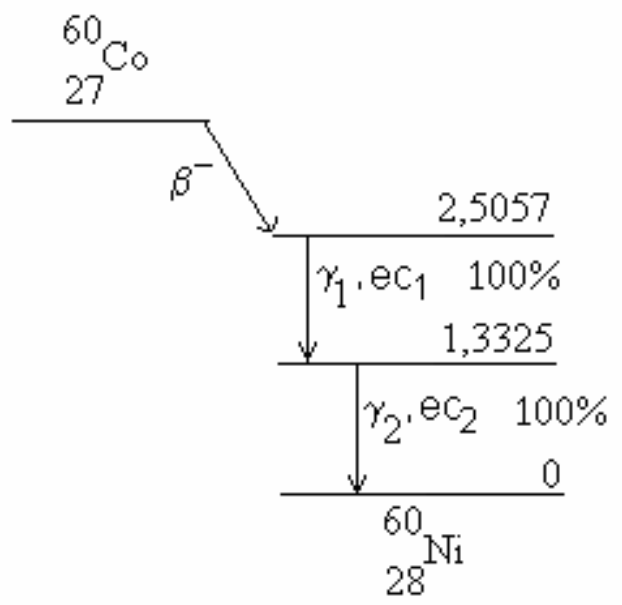

Figura 2.14 - Esquema de desintegração simplificado do ${ }^{60} \mathrm{Co}$.

$\mathrm{Na}$ tabela 2.4 são apresentadas as transições mais intensas das radiações emitidas do ${ }^{60} \mathrm{Co}$.

Foram preparados dois tipos de fontes radioativas para serem medidas no sistema $4 \pi(\mathrm{PC}) \beta-\gamma$ : fonte pura de ${ }^{60} \mathrm{Co}$ e fonte mista de ${ }^{35} \mathrm{~S}+{ }^{60} \mathrm{Co}$. 
Tabela 2.4 - Energias e probabilidades de emissão das transições mais intensas do ${ }^{60} \mathrm{Co}$.

\begin{tabular}{|c|c|c|c|c|}
\hline $\begin{array}{c}\text { Tipo de } \\
\text { Radiação }\end{array}$ & $\begin{array}{c}\text { Energia } \\
(\mathrm{keV})\end{array}$ & $\begin{array}{c}\text { Probabilidade de } \\
\text { emissão } \\
(\%)\end{array}$ & & \\
\hline \multicolumn{5}{|l|}{ Transição $\beta^{-}$} \\
\hline$\beta^{-}$ & $317,89 \pm 0,11$ & $99,92 \pm 0,03$ & & \\
\hline Transição $\gamma$ & & & $\alpha_{K}$ & $\alpha_{t}$ \\
\hline$\gamma_{1}$ & $1173,239 \pm 0,004$ & $99,91 \pm 0,02$ & $1,510,03$ & $1,67 \pm 0,04$ \\
\hline$\gamma_{2}$ & $1332,503 \pm 0,0005$ & $99,9989 \pm 0,0006$ & $1,15 \quad 0,03$ & $1,27 \pm 0,04$ \\
\hline
\end{tabular}

\subsection{O Método de Monte Carlo}

\subsubsection{Introdução}

A velocidade de processamento e a capacidade de memória dos modernos computadores tornaram possível a simulação de complicados problemas matemáticos utilizando o Método de Monte Carlo. O Método de Monte Carlo é um método numérico que permite resolver problemas físicos ou matemáticos, através da simulação de processos aleatórios. O Método de Monte Carlo pode ser considerado como uma metodologia matemática melhor do que a solução por meio de equações (geralmente regidas por leis de probabilidade conhecidas), que tornariam este tipo de problema tão complexo que não poderia ser expresso por meio de uma solução analítica simples ${ }^{[53-57]}$.

Considera-se como data de nascimento do Método de Monte Carlo o ano de 1949, em que apareceu o artigo com o título "The Monte Carlo methods"[53]. A criação deste método está ligado aos matemáticos norte-americanos J. von Neumann e S. Ulam, que foram os principais responsáveis pela grande utilização do método de Monte Carlo em Física e Engenharia modernas, sem a necessidade de fundamentos sofisticados da teoria estatística $^{[53]}$. 
O nome Monte Carlo é uma referência ao principado de Mônaco, célebre por seus cassinos. Um dos aparelhos mecânicos mais sensíveis que permitem obter variáveis aleatórias é a roleta.

Uma peculiaridade do método de Monte Carlo é que seu algoritmo tem uma estrutura muito simples. Como regra, elabora-se primeiro um programa para a realização de um evento aleatório. Depois esse evento se repete $\mathrm{N}$ vezes de modo que cada experiência seja independente das outras e toma-se a média dos resultados de todas as histórias.

A outra peculiaridade é que a incerteza estatística é, em geral, proporcional à $\sqrt{\frac{D}{N}}$, onde $\mathrm{D}$ é uma constante e $\mathrm{N}$ é o número de histórias. Esta fórmula permite observar-se que para diminuir o erro em 10 vezes é preciso aumentar $\mathrm{N}$ (ou seja, o número de processamentos) em 100 vezes.

Um mesmo problema pode ser resolvido aplicando variações distintas do método de Monte Carlo, para que correspondam a diferentes valores de D. Em diversos problemas consegue-se elevar consideravelmente a precisão dos resultados escolhendo um procedimento de cálculo que utiliza um valor pequeno de D.

\subsubsection{Problemas que Podem ser Resolvidos pelo Método de Monte Carlo}

O método de Monte Carlo permite simular qualquer processo cuja seqüência depende de fatores que sejam aleatórios e conhecidos. Em muitos problemas matemáticos que não tem a menor relação com questões aleatórias, pode-se inventar um modelo probabilístico artificial (ou mais de um modelo) que permita resolver estes problemas. Por conseguinte, pode-se dizer que o método de Monte Carlo é um método universal para a solução de problemas matemáticos. Historicamente, o método de Monte Carlo tem sido uma ferramenta computacional rotineira para problemas de transporte de partículas, com maior ênfase ao nêutron e ao fóton. 


\subsubsection{Números Aleatórios e Pseudo-Aleatórios}

O ponto crítico da aplicação do método de Monte Carlo é a geração de números distribuídos uniformemente no intervalo ]0, 1[ . Existem vários métodos para a geração desses números e a escolha do método a ser adotado é de grande importância, pois de seu sucesso depende a correta solução do problema.

Números aleatórios podem ser obtidos utilizando tabelas construídas através de procedimentos experimentais, como por exemplo, uma roleta de números. Entretanto, para a utilização de tabelas é necessário uma grande área de memória para armazenamento destes números, o que constitui uma desvantagem. Tão pouco é conveniente introduzir a tabela em uma memória externa e recorrer constantemente a ela já que isto afeta consideravelmente a velocidade de processamento. Usualmente utiliza-se fórmulas de recorrência que fornecem números chamados pseudo-aleatórios, uma vez que estes números são gerados deterministicamente.

O primeiro algoritmo destinado a contrução de números pseudo-aleatórios foi proposto por J. von Neumann e é conhecida como a técnica do quadrado central. Para explicá-lo vamos tomar um exemplo ${ }^{[53]}$.

Considere o número $v_{0}=0,9876$ formado por quatro algarismos. Ao elevarmos ao quadrado obtemos o número $v_{0}^{2}=0,97535376$ de oito algarismos. Tomemos os quatro algarismos que aparecem no centro deste número e consideramos o número $v_{1}=0,5353$.

Elevando agora $v_{1}$ ao quadrado $\left(v_{1}^{2}=0,28654609\right)$, escolhemos de novo os quatro algarismos do centro e consideremos $v_{2}=0,6546$. Procedendo da mesma forma, obteremos $\quad v_{2}^{2}=0,42850116, \quad v_{3}=0,8501 ; \quad v_{3}^{2}=0,72267001, \quad v_{4}=0,2670$; $v_{4}^{2}=0,07128900, v_{5}=0,1289$, etc. 
Podemos resumir este algoritmo na fórmula $v_{\mathrm{k}+1}=\mathrm{F}\left(v_{\mathrm{k}}\right)$, onde $\mathrm{F}$ representa o conjunto de operações que devemos realizar com o número $v_{\mathrm{k}}$ para obtermos $v_{\mathrm{k}+1}$. $\mathrm{O}$ número $v_{0}$ é um número dado.

As vantagens da utilização dos números pseudo-aleatórios são evidentes. Para obter um número basta realizar algumas operações simples, o que torna a velocidade de geração desses números aleatórios da mesma ordem de grandeza da velocidade dos computadores. O programa ocupa pouca memória e qualquer número $v_{\mathrm{k}}$ pode ser reproduzido facilmente. Apesar de ser impossível garantir que o algoritmo utilizado na geração de números aleatórios mantenha uma uniformidade desses números, basta comprovar uma vez a qualidade desta geração para poder aplicá-la depois reiteradamente na solução de problemas semelhantes.

Hoje, muitos computadores estão equipados com programas para esta finalidade. No presente trabalho utilizou-se o gerador de números aleatórios do programa FORTRAN (FORTRAN Visual Workbench Version 1.00). A eficácia do gerador foi verificada em simulações específicas, conforme descrito no capítulo 3.

\section{Exemplo Aplicado à Interação de Fótons Gama com a Matéria}

Considere na interação da radiação gama com a matéria apenas três processos, ou sejam: o efeito fotoelétrico (A), o efeito Compton (B) e o efeito de formação de pares (C). Supondo que probabilidades de ocorrer o evento do tipo A é de $0,2\left(\mathrm{p}_{1}\right)$, do tipo B é $0,5\left(\mathrm{p}_{2}\right)$ e do tipo $\mathrm{C}$ é $0,3\left(\mathrm{p}_{3}\right)^{[57]}$, tem-se que para um grande número $\mathrm{N}$ de números aleatórios (r) gerados:

$0,2 \mathrm{~N}$ estarão no intervalo $0<\mathrm{r}<0,2$

$0,5 \mathrm{~N}$ estarão no intervalo $0,2 \leq \mathrm{r}<0,7$

$0,3 \mathrm{~N}$ estarão no intervalo $0,7 \leq \mathrm{r}<1$

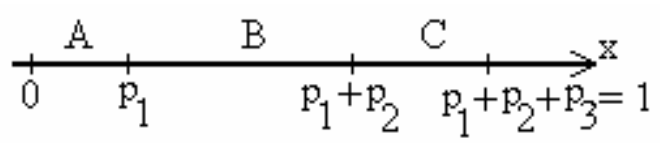


Cada vez que simulamos a experiência, geramos um número r. Se esse número está no intervalo correspondente ao evento A, então aceitamos como ocorrido o evento A. Se estiver no intervalo B, aceitamos B e assim sucessivamente.

O fluxograma do exemplo acima está esquematizado na figura 2.15 abaixo.

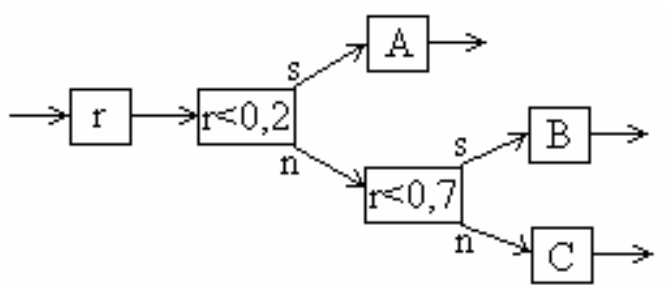

Figura 2.15 - Fluxograma para os eventos A, B e C.

Se $E_{1}, E_{2}, \ldots, E_{n}$ são eventos independentes mutuamente exclusivos, com probabilidades respectivamente $\mathrm{p}_{1}, \mathrm{p}_{2}, \ldots, \mathrm{p}_{\mathrm{n}}$, sendo $\mathrm{p}_{1}+\ldots+\mathrm{p}_{\mathrm{n}}=1$ e se $\mathrm{r}$ é um dos componentes de um conjunto de $\mathrm{N}$ números, o acontecimento do evento $\mathrm{E}_{\mathrm{i}}$ é determinado pela relação $\mathrm{p}_{1}+\ldots+\mathrm{p}_{\mathrm{i}-1} \leq \mathrm{r}<\mathrm{p}_{1}+\ldots+\mathrm{p}_{\mathrm{i}}$.

\subsection{Aplicação do Método de Monte Carlo ao Presente Trabalho}

As taxas de contagens nas vias beta, gama e de coincidência observadas pela técnica da extrapolação da eficiência na medida de atividade podem ser simuladas por meio da técnica de Monte Carlo. No presente trabalho, o cálculo de eficiências para fótons e elétrons foi efetuado por meio do programa MCNP4C - Monte Carlo N-Particle Transport Code System ${ }^{[58]}$, que é um programa consagrado e faz simulação para nêutron, elétron e fóton. Para simular todo o processo de detecção e calcular os espectros de energia depositada, o programa MCNP4C utiliza como dados de entrada: a) especificação da fonte radioativa; b) especificação dos tipos de superfície e definição de células para a geometria. Os resultados obtidos pelo programa foram utilizados para gerar uma tabela de resposta do detector proporcional $4 \pi$ para elétrons monoenergéticos e, uma tabela de resposta do detector $\mathrm{NaI}(\mathrm{Tl})$ para fótons monoenergéticos, utilizados no programa ESQUEMA. A simulação do processo de desintegração do radionuclídeo considerado foi efetuada por meio de um programa de Monte Carlo, desenvolvido no presente trabalho em linguagem 
FORTRAN (FORTRAN Visual Workbench Version 1.00), denominado ESQUEMA. A descrição deste programa é apresentada nas próximas seções.

\subsubsection{Período e Uniformidade na Geração de Números Aleatórios do Gerador de Números Aleatórios do FORTRAN.}

Inicialmente, foi feita uma análise da qualidade do gerador de números aleatórios do FORTRAN (FORTRAN Visual Workbench Version 1.00). Foi desenvolvido um código em linguagem fortran (APÊNDICE A) para verificar o período do gerador (quantidade de números gerados até a primeira repetição) de números aleatórios, e constatou-se que o período é da ordem de $2,1 \times 10^{9}$.

Desenvolveu-se também um outro código em linguagem fortran (APÊNDICE B) para verificar a uniformidade na geração de números aleatórios. $\mathrm{O}$ algoritmo consiste em gerar números aleatórios no intervalo de ]0, 1[. Em um primeiro nível, esse intervalo foi dividido em 20 subintervalos, e dentro de cada subintervalo gerou-se novos números aleatórios no intervalo de ]0, 1[, e dividiu-se esse subintervalo em 20 novos subintervalos e assim sucessivamente até 3 níveis. A divisão em 20 subintervalos e em 3 níveis garante que a geração de números aleatórios não ultrapassa o período do gerador. Os resultados obtidos podem ser vistos na figura $2.16,2.17$ e 2.18 .

O programa ESQUEMA necessita no máximo de 12 números aleatórios por história, em média, para os casos estudados no presente trabalho. Os resultados obtidos nos dois casos indicam que o período do gerador de números aleatórios é suficiente para o propósito do presente trabalho e a uniformidade na geração dos números aleatórios é confiável até $20^{3}$ subníveis, valor suficiente para as simulações apresentadas no presente trabalho. 


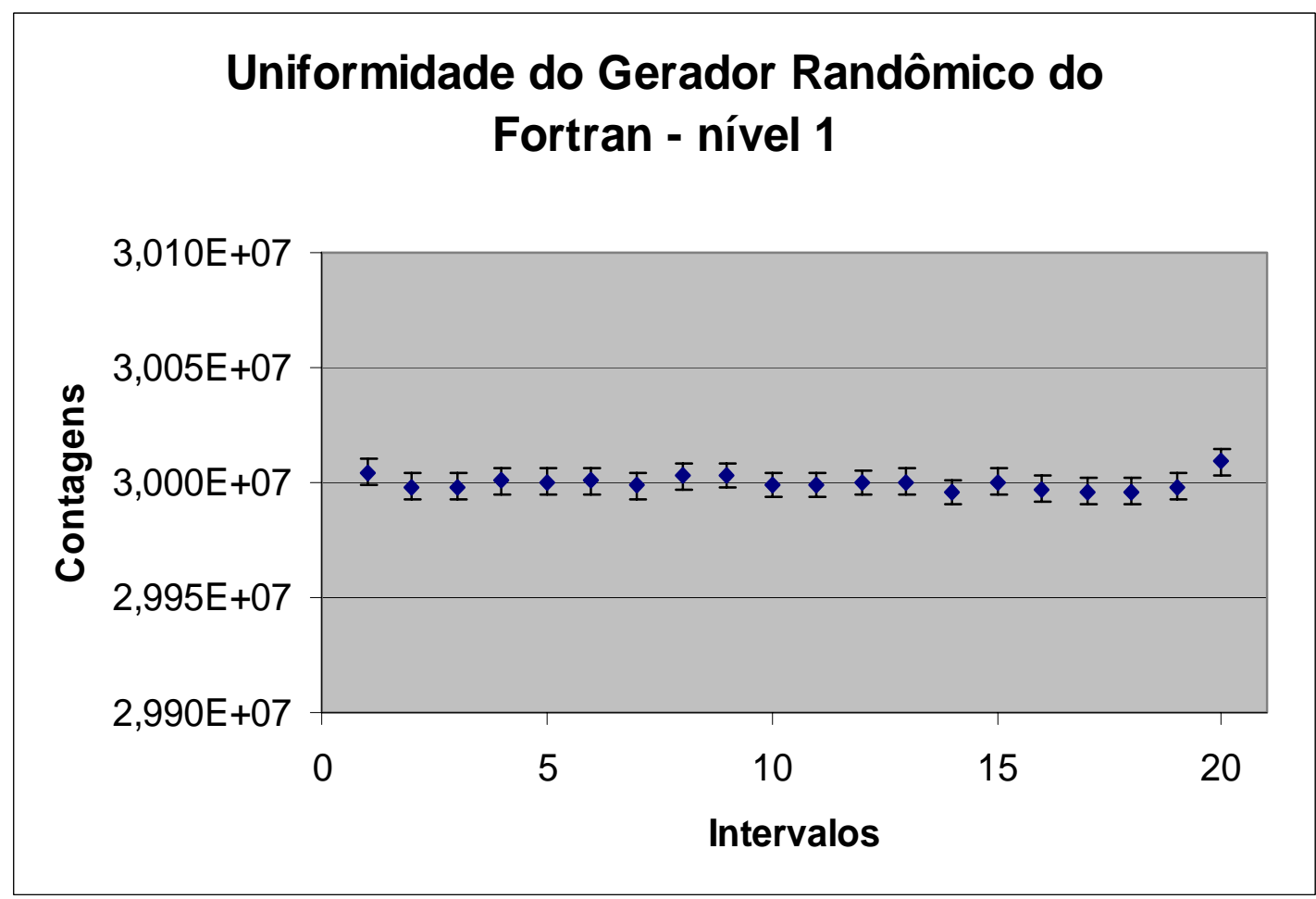

Figura 2.16 - Uniformidade do gerador de números aleatórios do Fortran, nível 1.

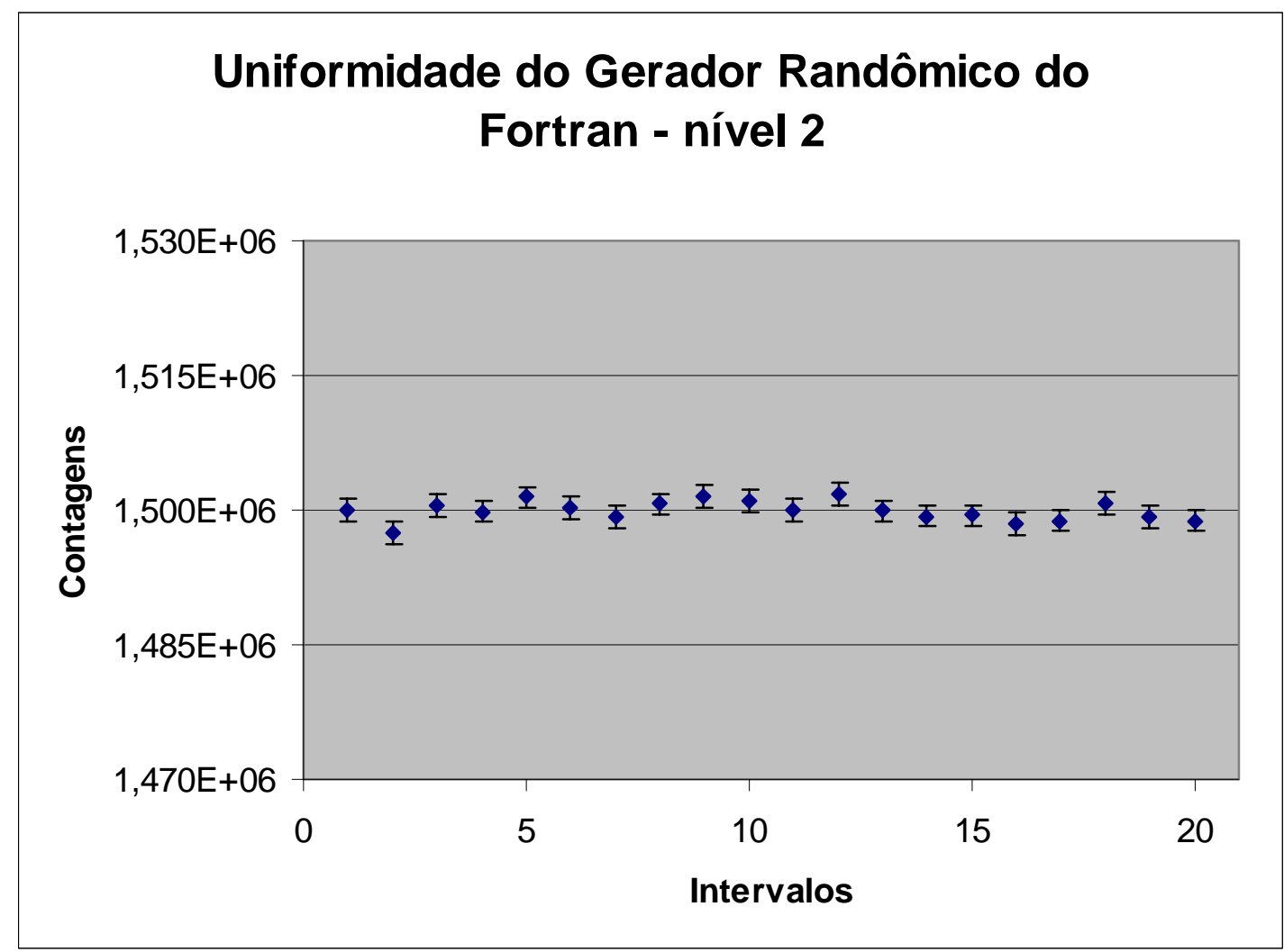

Figura 2.17 - Uniformidade do gerador de números aleatórios do Fortran, nível 2. 


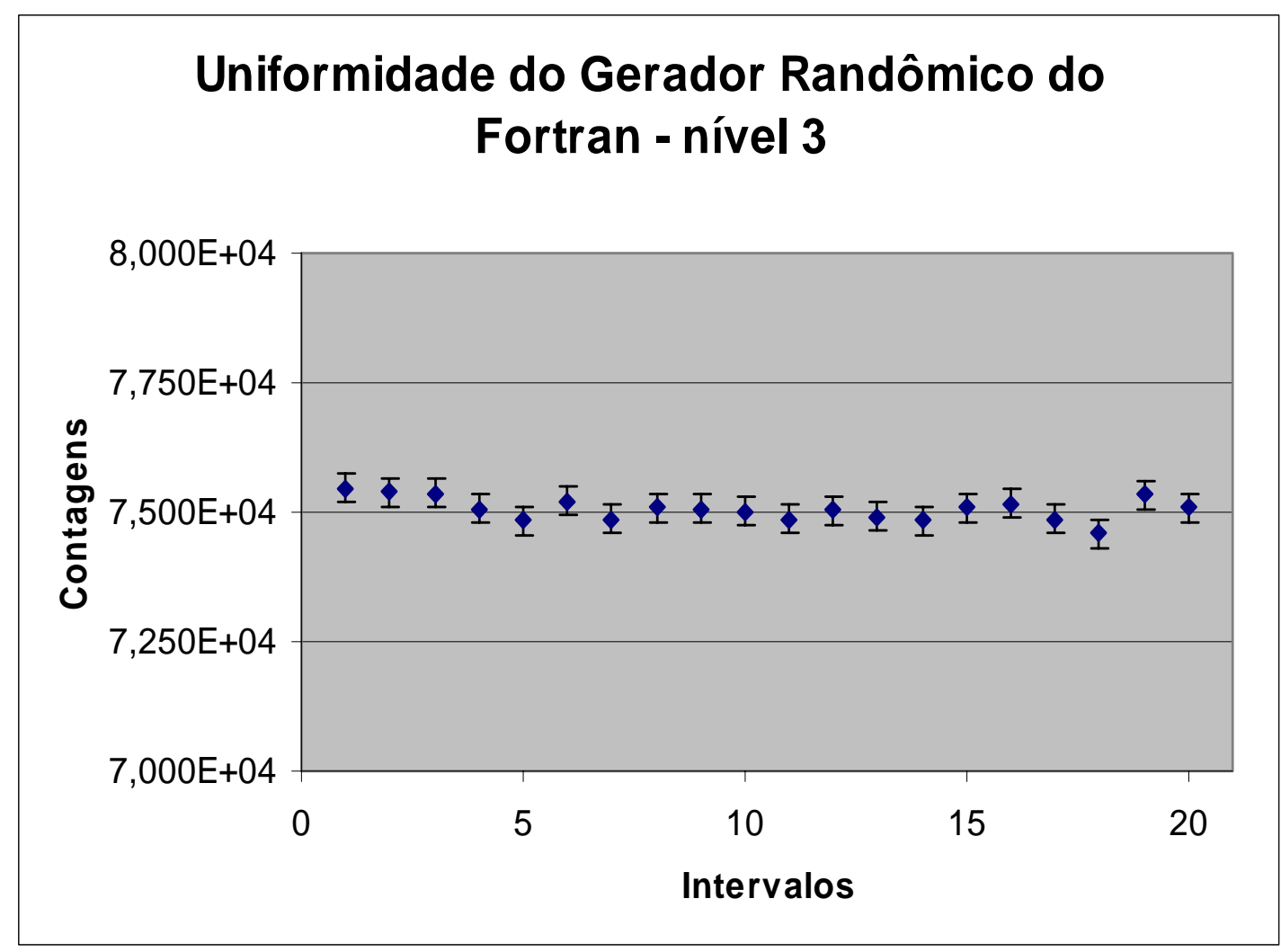

Figura 2.18 - Uniformidade do gerador de números aleatórios do Fortran, nível 3.

\subsubsection{Elaboração dos Dados de Entrada Utilizados no Programa ESQUEMA}

O programa ESQUEMA utiliza dois tipos de dados de entrada armazenados em arquivos. Um deles são dados constantes ou obtidos por intermédio de expressões analíticas ou da literatura e o outro obtido por meio do Método de Monte Carlo.

Os dados do arquivo de entrada para a modelagem do sistema utilizado pelo programa MCNP4C - Monte Carlo N-Particle Transport Code System para o cálculo do espectro de detecção para elétrons e fótons como função da energia foi determinado tomando-se por base informações da geometria do sistema de coincidência $4 \pi(\mathrm{PC}) \beta-\gamma$ do LMN (Figura 2.8).

O cálculo de espectros de energia depositada e eficiências para elétrons monoenergéticos foi efetuado por meio do programa de Monte Carlo MCNP4C, abrangendo o intervalo de energias entre $2,1650 \mathrm{keV}$ e 4,1024 MeV, para a geometria do arranjo experimental do detector proporcional $4 \pi$ utilizado no método da coincidência (figura 2.8). Esse intervalo foi dividido em subintervalos variando de $15 \%$ em energia. A 
menor energia que o programa de Monte Carlo MCNP4C fornece como resultado uma eficiência para elétrons monoenergéticos diferente de zero é $2,165 \mathrm{keV}$. Considerando que a energia depositada pelo beta é da ordem de $5 \mathrm{keV}$, o valor de 2,165 keV é relativamente alto. Para contornar essa restrição foi feito uma extrapolação das eficiências até a energia de $231 \mathrm{eV}$ considerando o mesmo comportamento da curva de 2,165 keV. Os resultados obtidos dão origem a um vetor de energias de $0 \mathrm{MeV}$ a 4,1024 MeV com energias variando conforme mencionado acima e uma tabela integrada e normalizada de eficiências para elétrons em função dessas energias. Esses dados estão dispostos no arquivo EFIBETA.DAT (APÊNDICE C)

Os espectros de energia depositada e eficiências para fótons também são determinadas por meio do programa MCNP4C. Análogo ao caso do elétron monoenergético, a menor energia que o programa de Monte Carlo MCNP4C fornece como resultado uma eficiência para fótons diferente de zero é $48 \mathrm{keV}$. Considerando esse fato, foi considerado o intervalo de energias de $48 \mathrm{keV}$ a $3,004 \mathrm{MeV}$, com subintervalos variando de $4 \mathrm{keV}$ em energia, para o detector de $\mathrm{NaI}(\mathrm{Tl})$, para a geometria do arranjo experimental utilizado no método de coincidências (figura 2.8). Por intermédio dos resultados obtidos pelo MCNP4C neste cálculo gerou uma tabela integrada e normalizada de eficiências para esses fótons em função da energia de $0 \mathrm{MeV}$ a 3,004 MeV com variação de energia conforme descrito acima. Essa tabela faz parte do arquivo EFIGAMA.DAT (APÊNDICE D). Esses resultados geraram também uma tabela de eficiência total em função da energia para esses fótons, cujos resultados estão no arquivo EFIGAMAT.DAT (APÊNDICE E).

Os dados do arquivo de entrada utilizados pelo programa ESQUEMA, como o valor da energia de corte dos elétrons emitidos e ou depositados no detector proporcional, número de canais, número de histórias, espessura do absorvedor, quantidade de absorvedores e a opção para definir se a desintegração ocorre por beta-gama, CE-gama ou emissão beta puro na qual é aplicada a técnica do traçador estão dispostos no arquivo CONSTANT.DAT. Um exemplo desse arquivo com a formatação e disposição desses dados e as respectivas definições está disponível no APÊNDICE F.

Os dados utilizados, provenientes das informações contidas no esquema de desintegração de um nuclídeo, obtidas na literatura, como níveis de energia, energia de 
desintegração (Q), intensidade dos ramos beta ou captura eletrônica, níveis de energia, probabilidade das transições gama, coeficientes de conversão interna, probabilidade de emissão de raio-X, estão dispostos no arquivo ESQUEMA.DAT. No APÊNDICE G está disponível um exemplo desse arquivo com a formatação e disposição desses dados e as respectivas definições.

Os dados referentes a escolha da janela gama, como quantidade de janelas e canais do intervalo de interesse estão dispostos no arquivo NPICOS.DAT, e no APÊNDICE H é apresentado um exemplo desse arquivo.

\subsubsection{Espectro Beta}

Para compor o espectro beta emitido do radionuclídeo, aplicou-se a Teoria de Fermi para desintegração $\beta^{[40]}$, levando-se em conta as características de energia e regras de seleção de momento angular para a transição nuclear considerada. Para o caso não relativístico a probabilidade de que um beta seja emitido com momento entre p e $(p+d p)$ é dada por ${ }^{[40]}$.

$$
\frac{\mathrm{dn}}{\mathrm{dE}_{0}}=\left(\frac{16 \pi^{2} \mathrm{~m}_{0}{ }^{5} \mathrm{c}^{4} \mathrm{dp}}{\mathrm{h}^{6}}\right) \eta^{2}\left(\mathrm{~W}_{0}-\mathrm{W}\right)^{2} \mathrm{~F}_{\mathrm{N}}(\mathrm{Z}, \eta) \mathrm{d} \eta
$$

na qual

$$
\begin{gathered}
\eta \equiv \frac{\mathrm{p}}{\mathrm{m}_{0} \mathrm{c}}, \\
\mathrm{W} \equiv \frac{\mathrm{E}+\mathrm{m}_{0} \mathrm{c}^{2}}{\mathrm{~m}_{0} \mathrm{c}^{2}}, \\
F_{N}(Z, \eta) \cong \frac{2 \pi y}{1-e^{-2 \pi y}}
\end{gathered}
$$

e o valor de y é dado por: 


$$
y \equiv \frac{ \pm Z}{137 \beta}= \pm Z \alpha \frac{W}{\eta} \quad\left\{\begin{array}{l}
+Z \text { para decaimento } \beta^{-} \\
-Z \text { para decaimento } \beta^{+}
\end{array}\right.
$$

Para uma verificação inicial, foi calculado um espectro para energia máxima beta de $1,0 \mathrm{MeV}$, supondo uma transição beta permitida. $\mathrm{O}$ intervalo foi dividido em 25 subintervalos de $40 \mathrm{keV}$, que foram integrados e normalizados. O espectro obtido é apresentado na figura 2.19 .

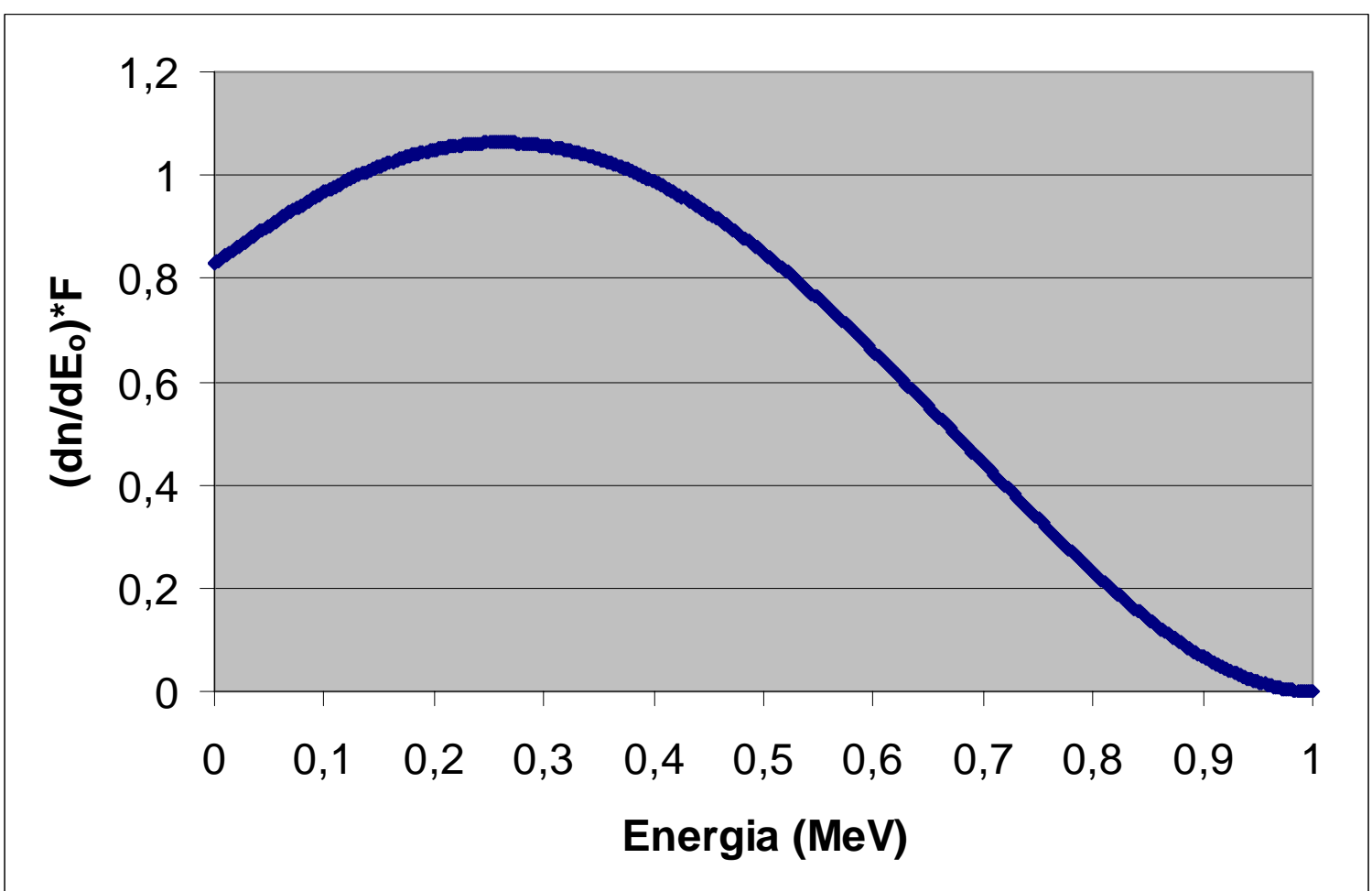

Figura 2.19 - Espectro beta para $\mathrm{E}=1 \mathrm{MeV}$ previsto pela Teoria de Fermi, para uma transição permitida

O programa ESQUEMA calcula o espectro beta para todas as energias dos ramos beta do esquema de desintegração utilizando a Teoria de Fermi vista acima. Valendo-se dos resultados obtidos constrói-se uma matriz que relaciona as probabilidades com as respectivas energias. Essa matriz está no arquivo FERMI.DAT (APÊNDICE I).

\subsubsection{Resolução em Energia}

O espectro do programa ESQUEMA é de alta resolução, entretanto a resolução em energia na maioria dos detectores cintiladores utilizados para radiação gama não é alta e, portanto, o espectro apresenta picos de energia relativamente alargados. Para simular o 
espectro experimental, no programa ESQUEMA, para o espectro de energia gama depositada, foi considerado a resolução relativa em energia $\mathrm{R}$ definida como $^{[42]}$ :

$$
R=\frac{F W H M}{H_{0}}=\frac{k}{\sqrt{E}}
$$

na qual:

FWHM = largura a meia altura do pico de energia.

$\mathrm{H}_{0}=$ altura de pulso médio correspondente ao mesmo pico.

$\mathrm{k}=$ constante de proporcionalidade

$\mathrm{E}=$ energia gama

O parâmetro R e a energia correspondente são dados de entrada, permitindo sua alteração de acordo com os parâmetros do sistema utilizado. No presente trabalho foi considerado o parâmetro $\mathrm{R}$ para a energia de $661,66 \mathrm{keV}$ do ${ }^{137} \mathrm{Cs}$, obtido experimentalmente no sistema de coincidências $4 \pi \beta-\gamma$ do LMN.

Na figura 3.1 e 3.5 pode-se observar os espectros gama simulados por Monte Carlo, não incluindo o efeito de resolução e incluindo o efeito de resolução.

\subsubsection{O Programa ESQUEMA}

O programa ESQUEMA foi desenvolvido no presente trabalho, para a simulação de todo o processo de desintegração radioativo considerado, acompanhando o esquema de desintegração desde o núcleo pai até o núcleo filho, em seu estado fundamental. Considera os níveis excitados de energia e analisa as transições gama ou elétrons de conversão interna, de modo a determinar as contagens nos canais beta, gama e coincidência. Um esquema do programa é mostrado na figura 2.20.

A presente versão do programa permite selecionar valores de corte para a energia das partículas beta emitidas e/ou para a energia depositada no detector $4 \pi \beta$ (correspondente a discriminação eletrônica), possibilitando assim a simulação da variação na eficiência beta. É possível também simular a variação da eficiência $\beta$ através do corte 


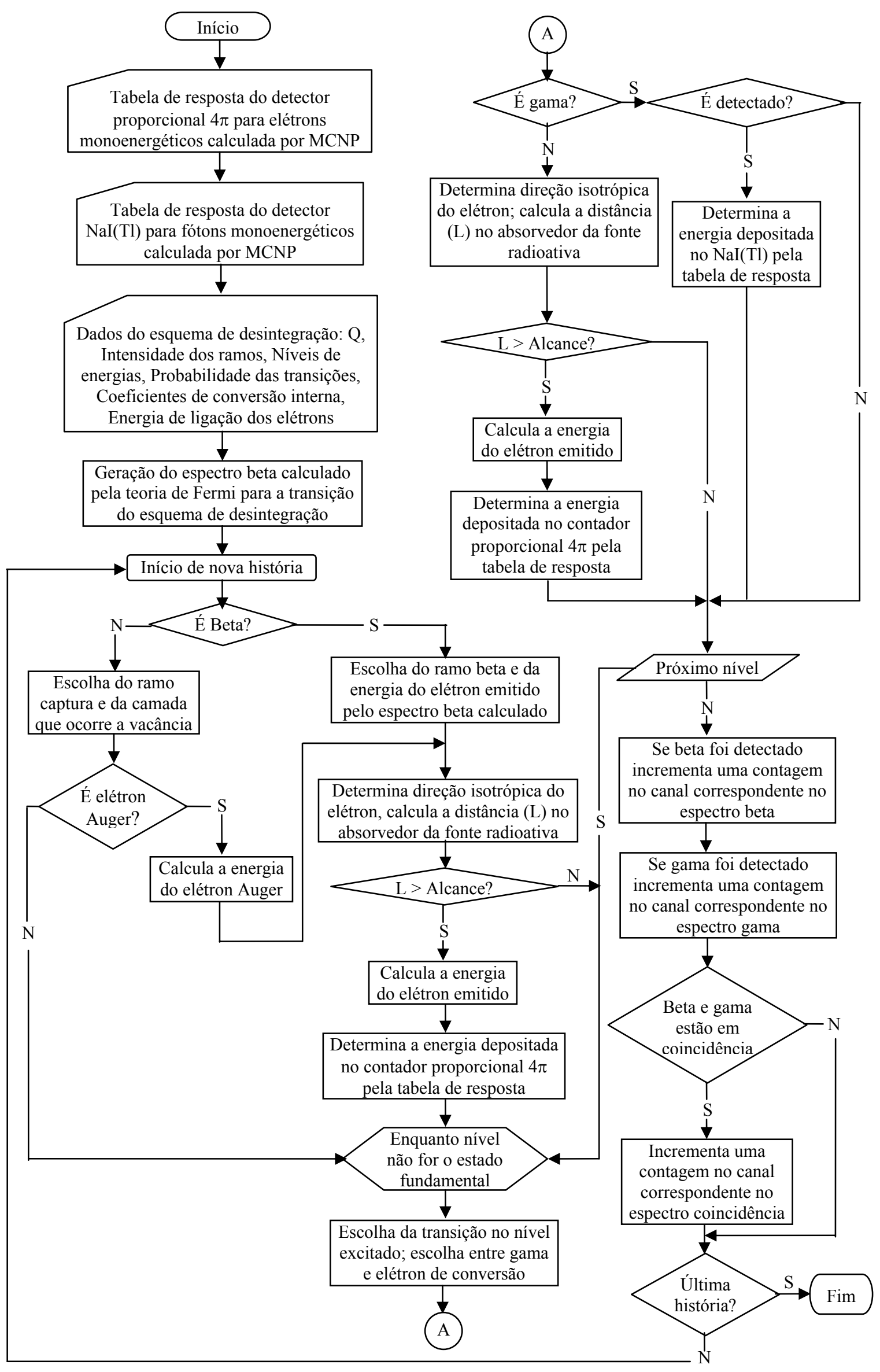

Figura 2.20 - Diagrama de Blocos do programa ESQUEMA 
para a energia depositada pelo uso de absorvedores externos de Collodion (nitrato de celulose) sobre o substrato da fonte radioativa.

O programa faz a simulação para desintegração por $\beta^{-}$, por Captura Eletrônica ou por desintegração mista $\beta^{-}$-CE, além de permitir o uso da Técnica do Traçador (seção 2.1.3.). A escolha do tipo de desintegração é feita através do sorteio de um número aleatório que é comparado com as probabilidades para $\beta^{-}$e Captura Eletrônica determinada pelo esquema de desintegração.

O programa permite fazer a simulação do sistema de coincidência $4 \pi \beta-\gamma$ utilizando o esquema de desintegração e prever qual a melhor transição gama, mesmo para esquema de desintegração complexo e determinar a atividade da fonte radioativa. $O$ programa ESQUEMA é apresentado no APÊNDICE J.

\subsubsection{Desintegração por Captura Eletrônica}

$\mathrm{Na}$ desintegração por Captura Eletrônica, o programa faz a escolha do ramo captura pelo sorteio de um número aleatório que é comparado com as probabilidades dos ramos captura, com base no esquema de desintegração do radionuclídeo.

A escolha da camada K, L etc, onde ocorre a vacância, é feita mediante o sorteio de um número aleatório, que é comparado com as probabilidades de captura do elétron dessas camadas $\left(\mathrm{P}_{\mathrm{K}}, \mathrm{P}_{\mathrm{L}}, \ldots\right)$ fornecida pelo esquema de desintegração do radionuclídeo. A escolha entre a emissão de raio $\mathrm{X}$ ou de um elétron Auger, que acompanha o preenchimento da vacância na camada $\mathrm{K}, \mathrm{L}, \ldots$, é feita por intermédio de outro número aleatório, que é comparada com $\omega_{\mathrm{K}}, \omega_{\mathrm{L}}, \ldots$, que são as probabilidades de emissão de raio $\mathrm{X}$.

A energia do raio $X_{K}$ emitido para uma transição $K X(X=L, M, N, \ldots)$ é calculada segundo a equação 1.31, e a energia do elétron Auger é calculada por meio da equação 1.32 . 
Ocorrendo a emissão de um elétron Auger, determina-se o alcance do elétron, para essa energia, através do sorteio de um número aleatório e compara-se com o alcance do elétron no absorvedor (Collodion) que se encontra tabelado no APÊNDICE K ${ }^{[59]}$.

Se o alcance do elétron for maior que o alcance tabelado, para essa energia, a escolha da energia do elétron depositada no detector proporcional $4 \pi$ é feita por meio do sorteio de um número aleatório, considerando os espectros de energia depositada para elétrons mono energéticos calculados previamente por Monte Carlo, conforme item 2.6.2.

\subsubsection{Desintegração por $\beta^{-}$}

No programa ESQUEMA, a escolha do ramo $\beta^{-}$é feita mediante o sorteio de um número aleatório e comparado com as probabilidades dos ramos beta, com base em dados obtidos no esquema de desintegração do radionuclídeo.

A energia do beta emitido é escolhida por meio do sorteio de um número aleatório e a sua probabilidade corresponde a um valor no espectro beta, determinado previamente pela Teoria de Fermi conforme item 2.6.3. para a energia do beta correspondente ao ramo escolhido anteriormente.

Calcula-se o alcance do beta para essa energia através do sorteio de um número aleatório e compara-se com o alcance no absorvedor (Collodion) tabelado no APÊNDICE $\mathrm{K}^{[59]}$.

Se o alcance do beta for maior que o alcance tabelado para essa energia, a escolha da energia do beta depositada no detector proporcional $4 \pi$ é feita por meio do sorteio de outro número aleatório, considerando os espectros de energia depositada para elétrons mono energéticos calculados previamente por Monte Carlo, conforme item 2.6.2.

\subsubsection{Transição $\gamma$ ou por Emissão de Elétrons de Conversão Interna}

Para as desintegrações por Captura Eletrônica ou por $\beta^{-}$, o programa segue o esquema de desintegração e a escolha da transição subseqüente gama ou por elétron de 
conversão também é feita por meio de um novo número aleatório. Esse número aleatório é comparado com a probabilidade de ocorrer uma transição gama $\frac{\alpha}{1+\alpha}$. Se esse número aleatório for menor do que a razão $\frac{\alpha}{1+\alpha}$ ocorreu uma transição gama, caso contrário ocorreu o elétron de conversão interna.

Ocorrendo uma transição gama, é sorteado um novo número aleatório e comparado com a eficiência total para a energia correspondente a essa transição, determinando se o gama foi detectado ou não detectado. Se foi detectado, a escolha da energia depositada no detector $\mathrm{NaI}(\mathrm{Tl})$ para essa transição é obtida com base em um novo número aleatório, considerando os espectros de energia depositada para fótons calculados previamente por Monte Carlo, conforme item 2.6.2.

Se ocorrer o elétron de conversão, determina-se o alcance do elétron conforme item 2.6.5.1 ou 2.6.5.2, e a escolha da energia depositada no detector proporcional $4 \pi$ para esse elétron é obtida por meio do sorteio de novo número aleatório, considerando os espectros de energia depositada para elétrons mono energéticos calculados previamente por Monte Carlo, conforme item 2.6.2.

Seguindo o esquema de desintegração, o programa verifica se está em um nível excitado ou no nível fundamental. Se estiver num nível excitado o programa seleciona nova transição gama conforme descrito anteriormente nesse item e executa esse processo até chegar ao nível fundamental.

\subsubsection{Espectros $\beta$, $\gamma$ e Coincidência}

Ocorrendo os eventos beta e gama, as contagens desses eventos são armazenadas nos respectivos espectros, de acordo com os canais correspondentes à energia depositada. Quando um evento gama é detectado em coincidência com um evento beta, uma contagem é incrementada no espectro de coincidências, no canal correspondente à energia gama depositada. Quando um elétron de conversão é detectado em coincidência com uma partícula beta, uma contagem é incrementada no espectro beta, no canal correspondente à energia cuja soma corresponde a das duas energias depositadas. 
Uma vez obtidos os três espectros de contagem: beta, gama e de coincidências,

determina-se $\frac{\mathrm{N}_{\beta} \mathrm{N}_{\gamma}}{\mathrm{N}_{\mathrm{c}}}$ e $\frac{1-\frac{\mathrm{N}_{\mathrm{c}}}{\mathrm{N}_{\gamma}}}{\frac{\mathrm{N}_{\mathrm{c}}}{\mathrm{N}_{\gamma}}}$, selecionando-se os intervalos de altura de pulso de interesse. É possível a obtenção de outros pontos, a partir da simulação da variação na eficiência beta por meio do corte para energia beta emitida e/ou detectada e/ou utilização de absorvedores externos sobre a fonte radioativa, possibilitando a construção do gráfico para determinar a taxa de desintegração $\mathrm{N}_{0}$ e o coeficiente angular da curva obtida por Monte Carlo.

\subsubsection{Determinação da Atividade pelo Método de Monte Carlo}

Para determinar o valor da atividade $\mathrm{N}_{0}$, foi utilizado o método dos mínimos quadrados combinando os dados experimentais e simulados. O valor de qui-quadrado correspondente é dado por:

$$
\chi^{2}=\left(\vec{y}_{\exp }-N_{0} \vec{y}_{M C}\right)^{T} V^{-1}\left(\vec{y}_{\exp }-N_{0} \vec{y}_{M C}\right)
$$

na qual:

$\vec{y}_{\exp } \quad$ é o vetor experimental de $\mathrm{N}_{\beta} \mathrm{N}_{\gamma} / \mathrm{N}_{c}$

$\vec{y}_{M C}$ é o vetor $\mathrm{N}_{\beta} \mathrm{N}_{\gamma} / \mathrm{N}_{\mathrm{c}}$ calculado por Monte Carlo para atividade unitária;

$\mathrm{N}_{0} \quad$ é a atividade específica da solução radioativa;

$\mathrm{V}$ é a matriz de covariância total, incluindo ambas as incertezas experimental e calculada, e

T representa matriz transposta. 


\subsubsection{Simulações Iniciais}

Foram realizados alguns cálculos preliminares de espectro de energia depositada e de eficiência utilizando o programa MCNP4C. O primeiro caso foi para a simulação de uma geometria simples (Figura 2.21), com o objetivo de comparar os resultados fornecidos pelo programa $\mathrm{MCNP} 4 \mathrm{C}$, com os obtidos utilizando uma fórmula analítica, que leva em conta apenas o ângulo sólido entre fonte e detector. Este resultado analítico corresponde ao valor esperado para o caso de fontes puntiformes e gamas de baixa energia, onde a deposição da radiação gama é próxima à superfície do detector.

Este procedimento foi efetuado considerando uma fonte gama puntiforme com energia de $50 \mathrm{keV}$ e um detector de $\mathrm{NaI}(\mathrm{Tl})$ cilíndrico com $2,53 \mathrm{~cm}$ de raio e altura de $4,96 \mathrm{~cm}$. Os resultados de eficiência são apresentados na Tabela 2.5:

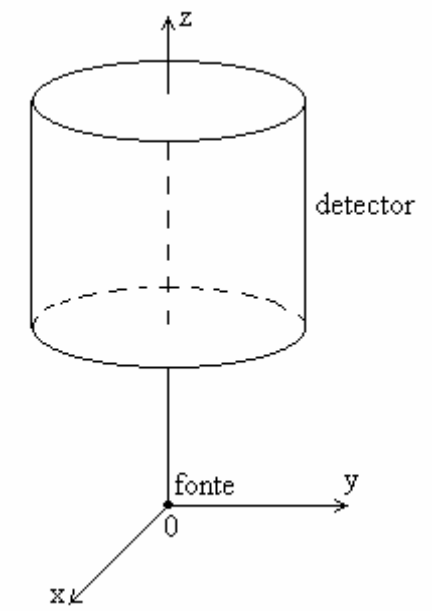

Figura 2.21 - Geometria simples para simulação pelo método de Monte Carlo no cálculo de eficiências.

Tabela 2.5 - Resultados de eficiências para geometria simples. Os números entre parênteses correspondem à incerteza nos últimos dígitos.

\begin{tabular}{|c|c|c|c|}
\hline Distância Fonte-Detector $(\mathrm{cm})$ & $\begin{array}{c}\text { Eficiência de Pico } \\
\left(\varepsilon_{\mathrm{p}}\right)\end{array}$ & $\begin{array}{c}\text { Eficiência Total } \\
\left(\varepsilon_{\mathrm{T}}\right)\end{array}$ & $\begin{array}{c}\text { Eficiência Analítica } \\
\left(\varepsilon_{\mathrm{T}}\right) \approx\left(\varepsilon_{\mathrm{p}}\right)\end{array}$ \\
\hline 0,001 & $0,4798(16)$ & $0,5011(16)$ & 0,50 \\
\hline 188 & $0,0000447(12)$ & $0,0000455(12)$ & 0,0000451 \\
\hline
\end{tabular}


Os resultados obtidos indicam que a modelagem e os resultados fornecidos pelo programa MCNP4C estão em bom acordo com os resultados analíticos dentro da incerteza estatística, objetivo desta simulação.

O segundo caso foi a simulação para geometria simplificada (Figura 2.8), do arranjo experimental utilizado no LMN, com o intuito de comparar os resultados obtidos por Monte Carlo para essa modelagem com resultados experimentais para essa geometria.

Os resultados obtidos com o programa MCNP4C para as eficiências gama total e de pico do cintilador de $\mathrm{NaI}(\mathrm{Tl})$, correspondentes ao fóton de $661,66 \mathrm{keV}$ do ${ }^{137} \mathrm{Cs}$ são comparados na Tabela 2.6, com as eficiências experimentais obtidas por meio de uma fonte calibrada pela Agência Internacional de Energia Atômica.

Foi efetuada também uma simulação para a eficiência de pico para o fóton de $1173,238 \mathrm{keV}$ do ${ }^{60} \mathrm{Co}$ e comparada com a eficiência de pico experimental. A eficiência total é de difícil determinação experimental para as energias individuais do ${ }^{60} \mathrm{Co}$, utilizando um detector de $\mathrm{NaI}(\mathrm{Tl})$. Por esta razão, foi efetuada uma simulação para eficiência total para a energia média de $1252,5 \mathrm{keV}$, para comparar com a eficiência total experimental.

Os resultados obtidos estão incluídos na Tabela 2.6.

Tabela 2.6 - Eficiência gama para o fóton de $661,66 \mathrm{keV}$ do ${ }^{137} \mathrm{Cs}$ e eficiências de pico para o fóton de $1173,238 \mathrm{keV}$ e total, para energia média de $1252,5 \mathrm{keV}$ do ${ }^{60} \mathrm{Co}$.

\begin{tabular}{|c|c|c|c|}
\hline \multirow{2}{*}{ Nuclídeo } & Eficiência & Monte Carlo & Experimental \\
\hline \multirow{2}{*}{${ }^{137} \mathrm{Cs}$} & Pico & $0,0349(6)$ & $0,0334(4)$ \\
\cline { 2 - 4 } & Total & $0,0897(14)$ & $0,0908(11)$ \\
\hline \multirow{2}{*}{${ }^{60} \mathrm{Co}$} & Pico & $0,0230(5)$ & $0,0236(4)$ \\
\cline { 2 - 4 } & Total & $0,0735(15)$ & $0,0739(10)$ \\
\hline
\end{tabular}

A boa concordância entre os resultados obtidos por Monte Carlo e a Experimental indica que a modelagem se aproxima da experimental. 
A verificação da simulação do método de coincidência pelo método de Monte Carlo por meio do programa ESQUEMA, foi efetuada a partir de um esquema de desintegração hipotético simples, apresentado na Figura 2.22, no qual o coeficiente angular da curva resultante é fácil de se obter analiticamente.

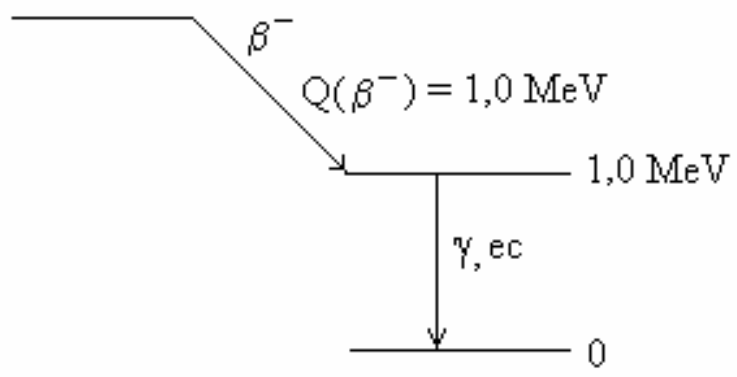

Figura 2.22 - Esquema de desintegração hipotético simples.

Nesse esquema hipotético simples, um único ramo beta com energia de $1,0 \mathrm{MeV}$ é seguido por um único fóton gama de 1,0 MeV. Esta transição gama é parcialmente convertida internamente com um coeficiente de conversão $(\alpha)$ igual a 0,1 . A energia do elétron de conversão é de $0,95 \mathrm{MeV}$. Nessas condições, as equações de coincidência serão:

$$
\begin{gathered}
\mathrm{N}_{\beta}=\mathrm{N}_{0} \varepsilon_{\beta}+\mathrm{N}_{0}\left(1-\varepsilon_{\beta}\right) \frac{\alpha}{1+\alpha} \varepsilon_{\mathrm{ec}} \\
\mathrm{N}_{\gamma}=\mathrm{N}_{0} \varepsilon_{\gamma} \frac{1}{1+\alpha} \\
\mathrm{N}_{\mathrm{C}}=\mathrm{N}_{0} \varepsilon_{\beta} \varepsilon_{\gamma} \frac{1}{1+\alpha}
\end{gathered}
$$

assim,

$$
\frac{\mathrm{N}_{\beta} \mathrm{N}_{\gamma}}{\mathrm{N}_{\mathrm{C}}}=\mathrm{N}_{0}\left[1+\frac{\left(1-\varepsilon_{\beta}\right)}{\varepsilon_{\beta}} \frac{\alpha}{1+\alpha} \varepsilon_{\mathrm{ec}}\right]
$$


Por intermédio da equação (2.26) pode-se observar que há uma dependência linear entre $\frac{\mathrm{N}_{\beta} \mathrm{N}_{\gamma}}{\mathrm{N}_{\mathrm{C}}}$ e o parâmetro de ineficiência $\frac{\left(1-\varepsilon_{\beta}\right)}{\varepsilon_{\beta}}$, e a declividade corresponde a $\frac{\alpha}{1+\alpha} \varepsilon_{\mathrm{ec}}$. Enquanto a eficiência de detecção do elétron de conversão permanecer constante e próxima de $100 \%$, a curva será linear. No esquema de desintegração proposto, a declividade esperada é igual a 0,0909.

A Figura 2.23 mostra a curva de extrapolação obtida pelo Método de Monte Carlo. A curva $A$ corresponde à variação na eficiência beta, obtida alterando-se o valor de corte na energia beta emitida, enquanto a curva $B$ corresponde à variação na eficiência beta, alcançada modificando-se o valor de corte para a energia beta depositada.

Observa-se um comportamento não-linear para a curva $\mathrm{B}$, na região de baixas eficiências. Isto pode ser explicado, porque nesta região, os elétrons de conversão começam a ser absorvidos e deixam de contribuir na contagem do detector beta.

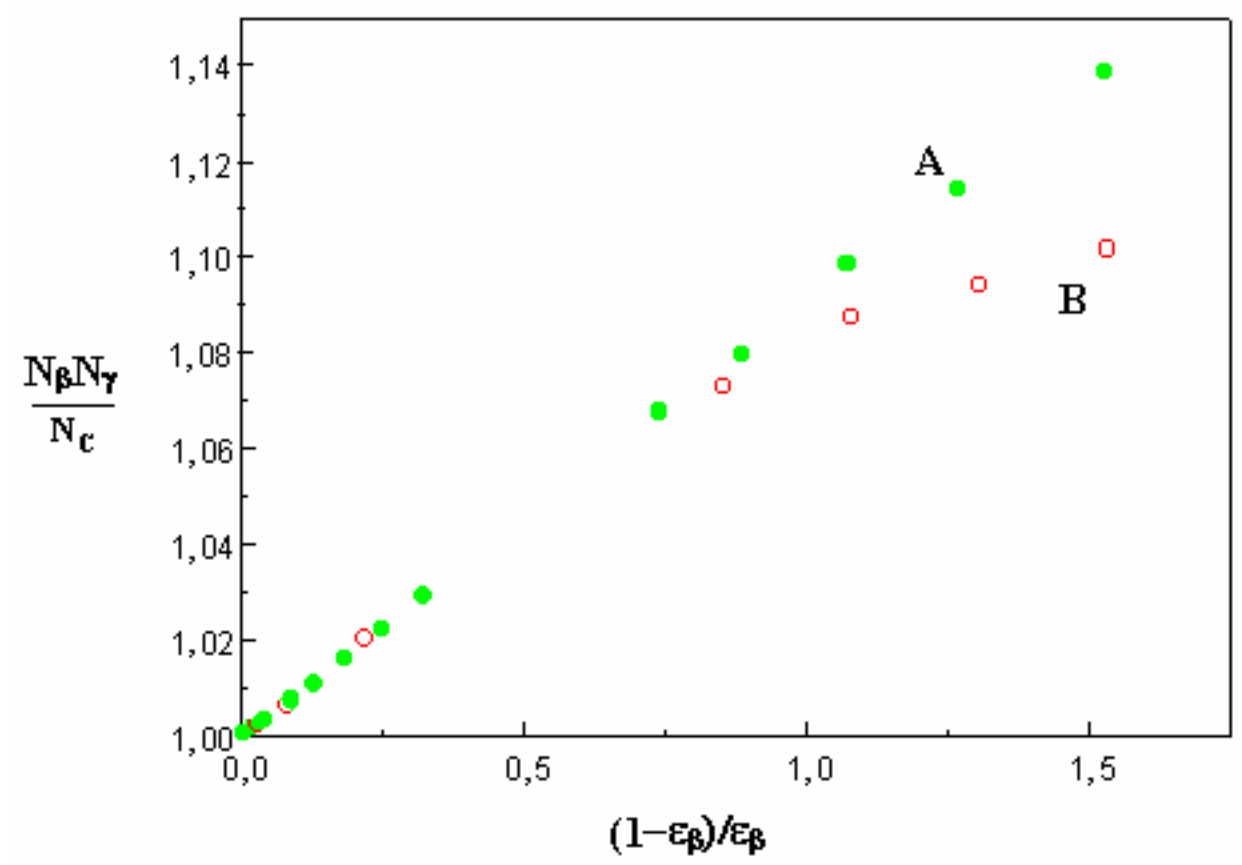

Figura 2.23 - Curvas de extrapolação previstas pelo método de Monte Carlo 
As duas curvas estão de acordo na região de alta eficiência e o ajuste pelo método dos mínimos quadrados resultou na inclinação esperada $(0,0909)$ dentro da incerteza estatística para ambas as curvas. Para abscissa acima de 1,0 (eficiência beta abaixo de 50\%), a curva B decresce, indicando que a eficiência do elétron de conversão está diminuindo para valores abaixo de 100\%. 


\section{RESULTADOS E DISCUSSÃO}

Neste capítulo são apresentados e discutidos os resultados obtidos na padronização de radionuclídeos por maio do sistema de coincidência $4 \pi \beta-\gamma$ aplicando o método de Monte Carlo. Os radionuclídeos estudados foram: ${ }^{134} \mathrm{Cs},{ }^{72} \mathrm{Ga}$, que desintegram por $\beta^{-} ;{ }^{133} \mathrm{Ba}$, que desintegra por captura eletrônica e ${ }^{35} \mathrm{~S}$, emissor beta puro. Para este último foi aplicada a técnica do traçador, sendo utilizado o ${ }^{60} \mathrm{Co}$ como traçador.

\subsection{Padronização do ${ }^{134} \mathrm{Cs}$}

A figura 3.1 apresenta o espectro gama simulado por Monte Carlo para o detector de $\mathrm{NaI}(\mathrm{Tl})$, de acordo com a geometria do sistema $4 \pi$ (PC) $\beta-\gamma$. No espectro A o efeito de resolução não está incorporado e mostra a contribuição de todas as transições gama. No espectro B este efeito foi incorporado, de acordo com a equação 2.21 , de modo a ajustar-se com o espectro experimental na energia de $661,66 \mathrm{keV}$, que corresponde a energia gama do ${ }^{137} \mathrm{Cs}$.

A figura 3.2 apresenta o comportamento de $\frac{N_{\beta} N \gamma}{N_{C}}$ como função do parâmetro de ineficiência $\frac{1-\frac{N_{C}}{N_{\gamma}}}{\frac{N_{C}}{N_{\gamma}}}$ para o ${ }^{134}$ Cs. Os círculos e triângulos cheios correspondem aos resultados experimentais para as janelas gama de $600 \mathrm{keV}$ e $800 \mathrm{keV}$ respectivamente, obtidos com a utilização de absorvedores externos de collodion sobre a fonte radioativa. Os círculos e triângulos vazios correspondem aos resultados obtidos pelo programa ESQUEMA utilizando o método de Monte Carlo, e simulando a utilização de absorvedores externos de collodion. 


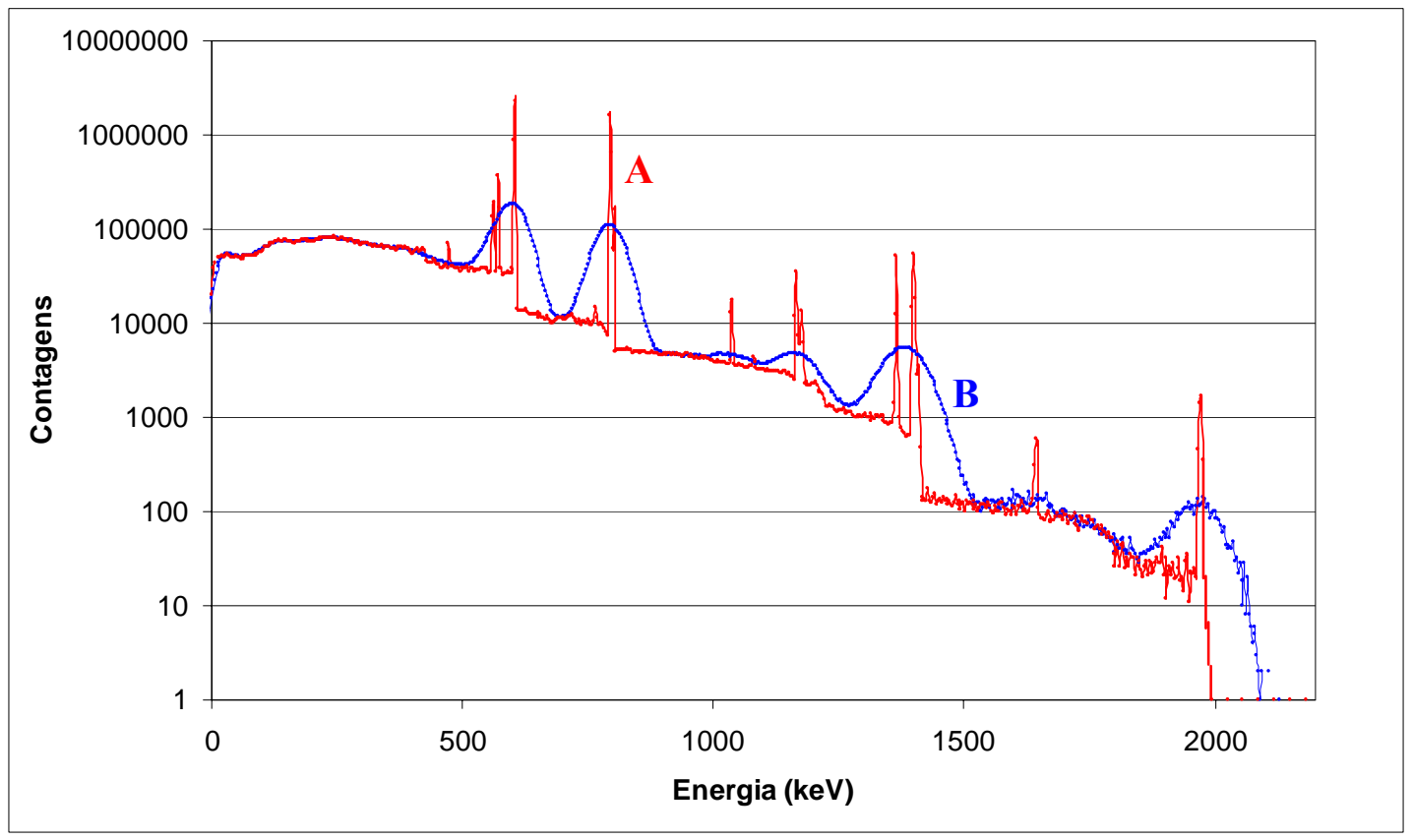

Figura 3.1 - Espectro gama simulado por Monte Carlo para o ${ }^{134} \mathrm{Cs}$. Espectro A não inclui efeito de resolução. Espectro B inclui efeito de resolução ajustado ao espectro experimental para energia de $661,66 \mathrm{keV}$ do ${ }^{137} \mathrm{Cs}$.

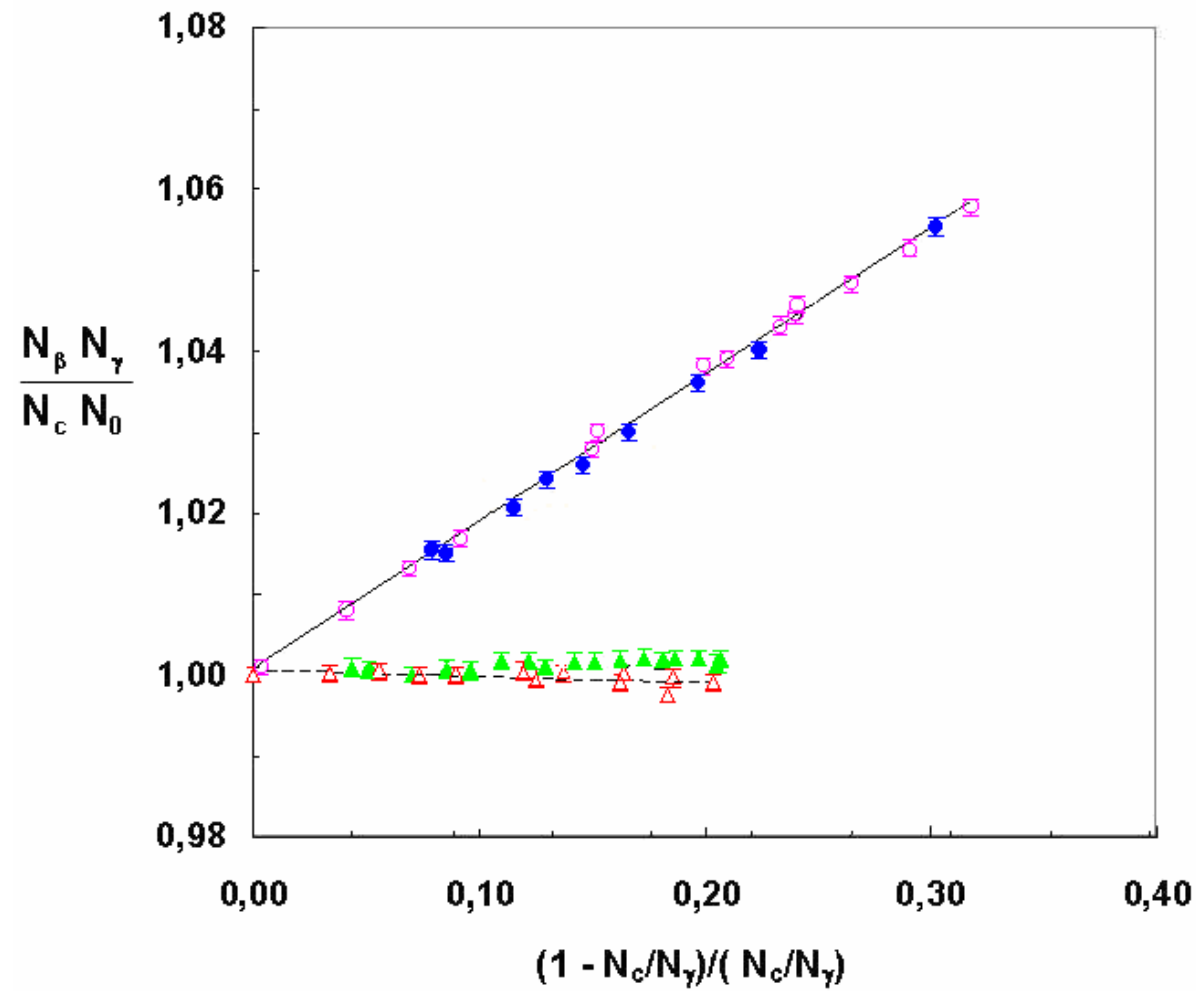

Figura 3.2 - Comportamento de $\mathrm{N}_{\beta} \mathrm{N}_{\gamma} / \mathrm{N}_{\mathrm{C}} \mathrm{N}_{0}$ em função do parâmetro de ineficiência $\left(1-\mathrm{N}_{\mathrm{C}} / \mathrm{N}_{\gamma}\right) /\left(\mathrm{N}_{\mathrm{C}} / \mathrm{N}_{\gamma}\right)$. Pontos superiores correspondem à janela gama de $600 \mathrm{keV}$ e pontos inferiores correspondem à janela gama de $800 \mathrm{keV}$. Símbolos cheios correspondem a resultados experimentais e símbolos vazios a resultados por Monte Carlo. 
$\mathrm{Na}$ padronização do radionuclídeo ${ }^{134} \mathrm{Cs}$, que apresenta esquema de desintegração complexo por beta-gama podemos observar as curvas de extrapolação apresentadas na figura 3.2 na qual pode-se verificar que há um bom acordo entre a declividade experimental $(0,1819(30))$ e a calculada por Monte Carlo $(0,1783(24))$, para a janela gama de $600 \mathrm{keV}$. Esta grande declividade pode ser explicada pela contribuição das transições de 569,32 keV e 801,93 keV na janela gama de $600 \mathrm{keV}$. Essas transições são provenientes da transição beta de baixa energia (máxima energia de $88,5 \mathrm{keV}$ ) para a qual as partículas beta são absorvidas mais facilmente em comparação com o beta principal (máxima energia de 657,8 keV). A janela de $800 \mathrm{keV}$ é muito menos afetada pelo ramo beta de baixa energia resultando numa declividade pequena. $\mathrm{O}$ valor experimental para a declividade em $800 \mathrm{keV}$ foi $(0,0099(19))$ e por Monte Carlo foi $(-0,0041(36))$. Esta pequena diferença pode ser explicada por uma possível subestimação da eficiência gama do detector beta feita por Monte Carlo. Contudo o efeito na atividade extrapolada é inferior a $0,1 \%$ para eficiências beta maiores que $94 \%$.

\subsection{Padronização do ${ }^{72}$ Ga}

A atividade do ${ }^{72} \mathrm{Ga}$ que também desintegra por beta-gama foi obtida pelo ajuste dado pela equação (2.22). A tabela 3.1 mostra os resultados para as duas janelas gama selecionadas. A atividade resultante é a média entre os resultados das duas janelas gama.

As figuras 3.3 e 3.4 mostram as curvas dos resultados experimentais $\frac{\mathrm{N}_{\beta} \mathrm{N}_{\gamma}}{\mathrm{N}_{\mathrm{C}} \mathrm{N}_{0}}$ como função de $\frac{1-\frac{N_{C}}{N_{\gamma}}}{\frac{N_{C}}{N_{\gamma}}}$ comparadas com os resultados obtidos na simulação por Monte Carlo (MC). O melhor ajuste para a janela 1 foi uma constante e para a janela 2 foi uma reta. 
Tabela 3.1 - Parâmetros obtidos para as duas janelas gama selecionadas utilizando a simulação por Monte Carlo.

\begin{tabular}{|c|c|c|c|c|c|c|}
\hline \multirow{2}{*}{ Fonte } & \multicolumn{2}{|c|}{ Janela 1 } & \multicolumn{2}{c|}{ Janela 2 } & \multicolumn{2}{c|}{ Média } \\
\cline { 2 - 7 } & \multicolumn{2}{|c|}{ Atividade (Bq) } & \multicolumn{2}{c|}{ Atividade (Bq) } & \multicolumn{2}{c|}{ Atividade (Bq) } \\
\cline { 2 - 7 } & Monte Carlo & Experimental & Monte Carlo & Experimental & Monte Carlo & Experimental \\
\hline Fonte 1 & $3877 \pm 6$ & $3882 \pm 10$ & $3861 \pm 9$ & $3860 \pm 31$ & $3869 \pm 5$ & $3871 \pm 10$ \\
\hline Fonte 2 & $3871 \pm 6$ & $3875 \pm 9$ & $3866 \pm 6$ & $3889 \pm 9$ & $3869 \pm 4$ & $3881 \pm 7$ \\
\hline Fonte 3 & $11546 \pm 21$ & $11578 \pm 60$ & $11446 \pm 21$ & $11440 \pm 61$ & $11496 \pm 20$ & $11509 \pm 41$ \\
\hline Fonte 4 & $41447 \pm 203$ & $41536 \pm 220$ & $41226 \pm 198$ & $41391 \pm 206$ & $41336 \pm 142$ & $41463 \pm 149$ \\
\hline Declividade & $0,0137 \pm 0,0007$ & 0 & $0,223 \pm 0,003$ & $0,225 \pm 0,018$ & & \\
\hline
\end{tabular}

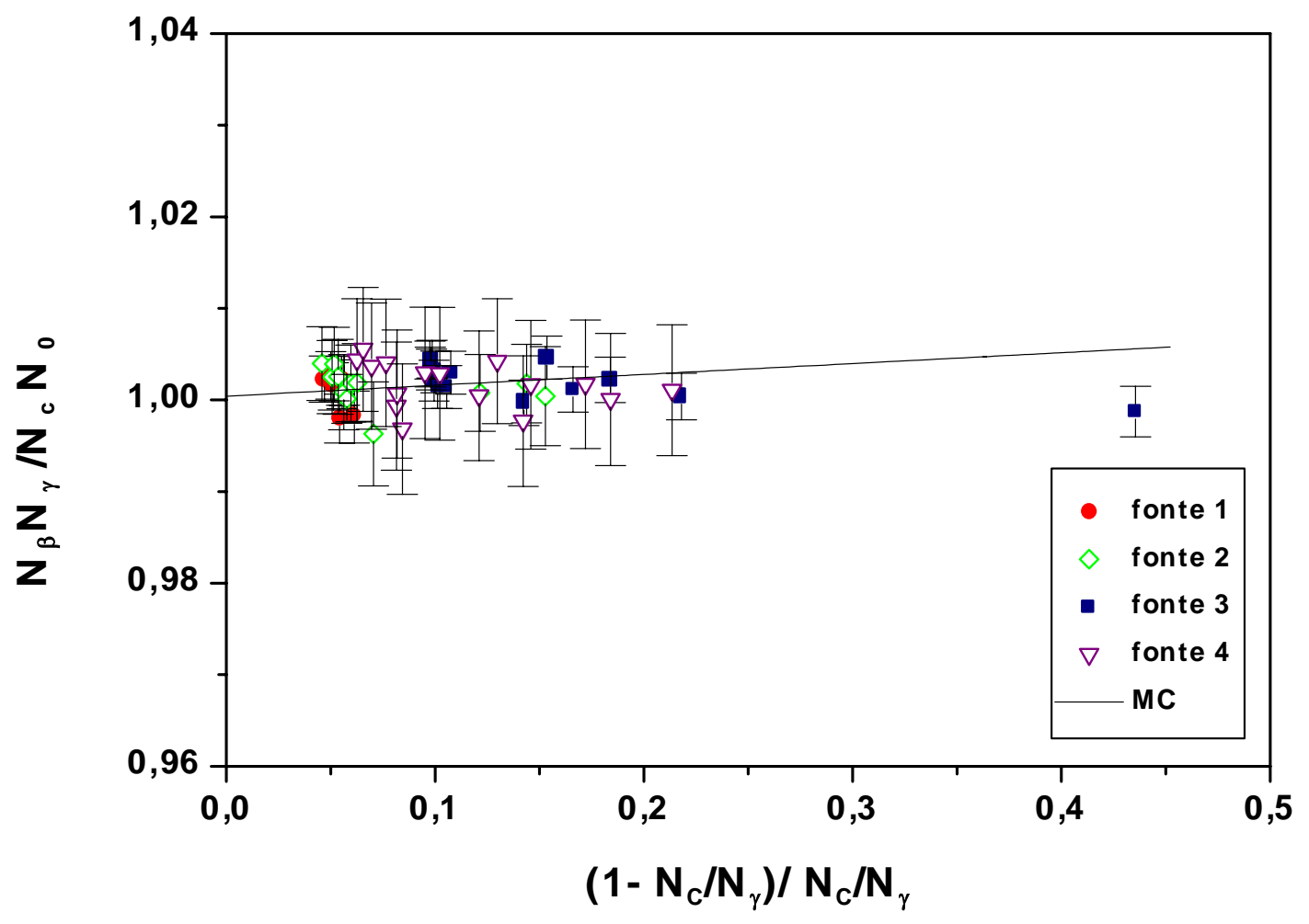

Figura 3.3 - Curvas dos resultados experimentais comparadas com a simulação por Monte Carlo (Janela 1) 


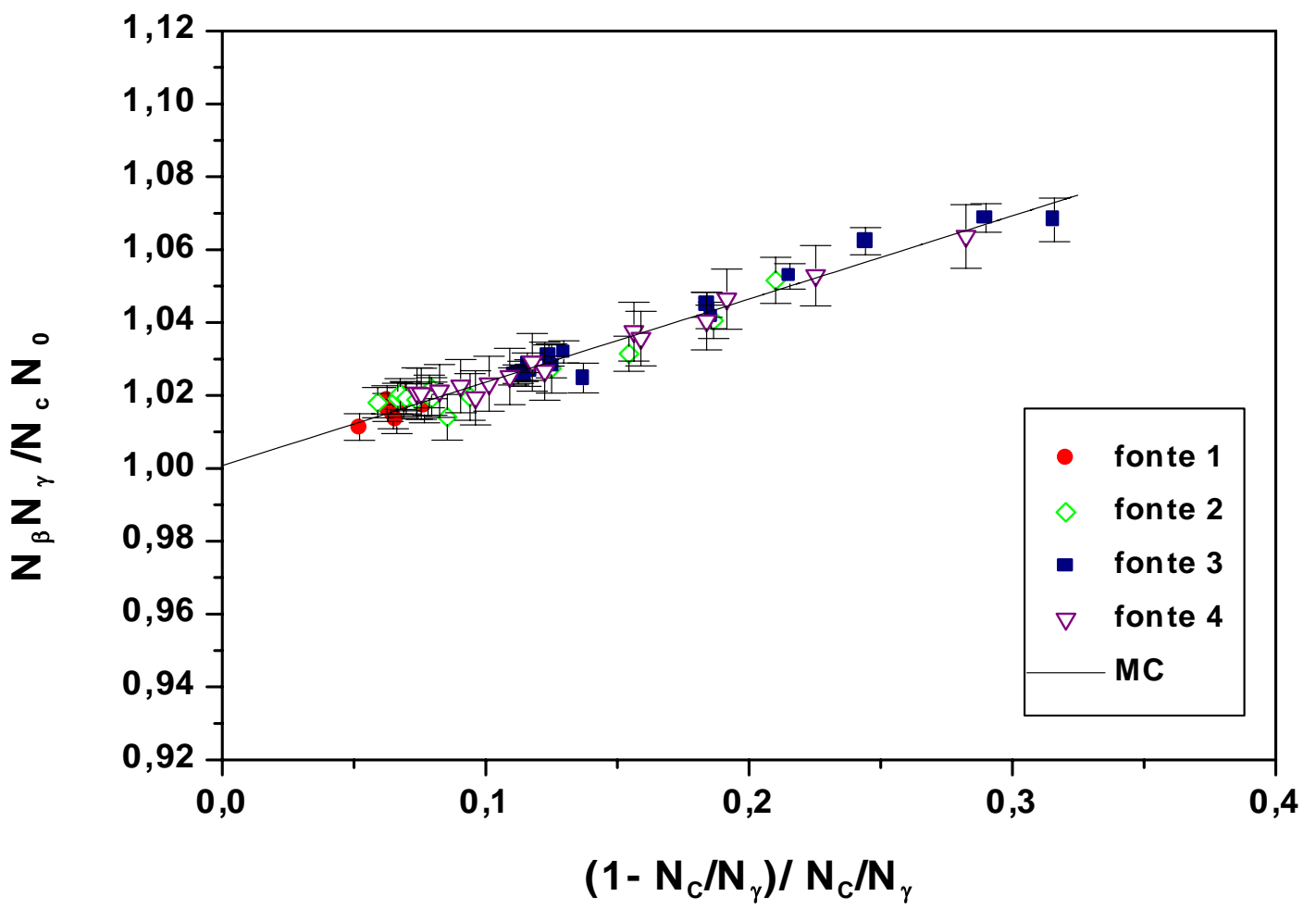

Figura 3.4 - Curvas dos resultados experimentais comparadas com a simulação por Monte Carlo (Janela 2)

Para a padronização do ${ }^{72} \mathrm{Ga}$, os resultados experimentais estão de acordo com os obtidos por meio da simulação de Monte Carlo (figuras 3.3 e 3.4). Isto indica que, nos casos onde temos poucos pontos experimentais que dificultam a extrapolação, o resultado obtido por Monte Carlo pode ser usado para atingir uma boa exatidão.

\subsection{Padronização do ${ }^{133} \mathrm{Ba}$}

$\mathrm{O}{ }^{133} \mathrm{Ba}$ se desintegra por captura eletrônica e tem esquema de desintegração complexo, apresentando dois ramos de captura e várias transições gama (figura 2.12). A janela gama experimental foi ajustada para abranger o intervalo de energias entre $222 \mathrm{keV}$ e $420 \mathrm{keV}$.

A figura 3.5 mostra dois espectros gama do ${ }^{133} \mathrm{Ba}$ simulado por Monte Carlo para o $\mathrm{NaI}(\mathrm{Tl})$ de acordo com a geometria do sistema $4 \pi(\mathrm{PC}) \beta-\gamma$. No espectro A não está incorporado o efeito de resolução e podem ser visualizadas as contribuições de todas as 
transições gama. $\mathrm{O}$ espectro $\mathrm{B}$ o efeito de resolução para o $\mathrm{NaI}(\mathrm{Tl})$ foi incorporado e foi ajustado ao espectro experimental para a energia gama de $661,66 \mathrm{keV}$ do ${ }^{137} \mathrm{Cs}$.

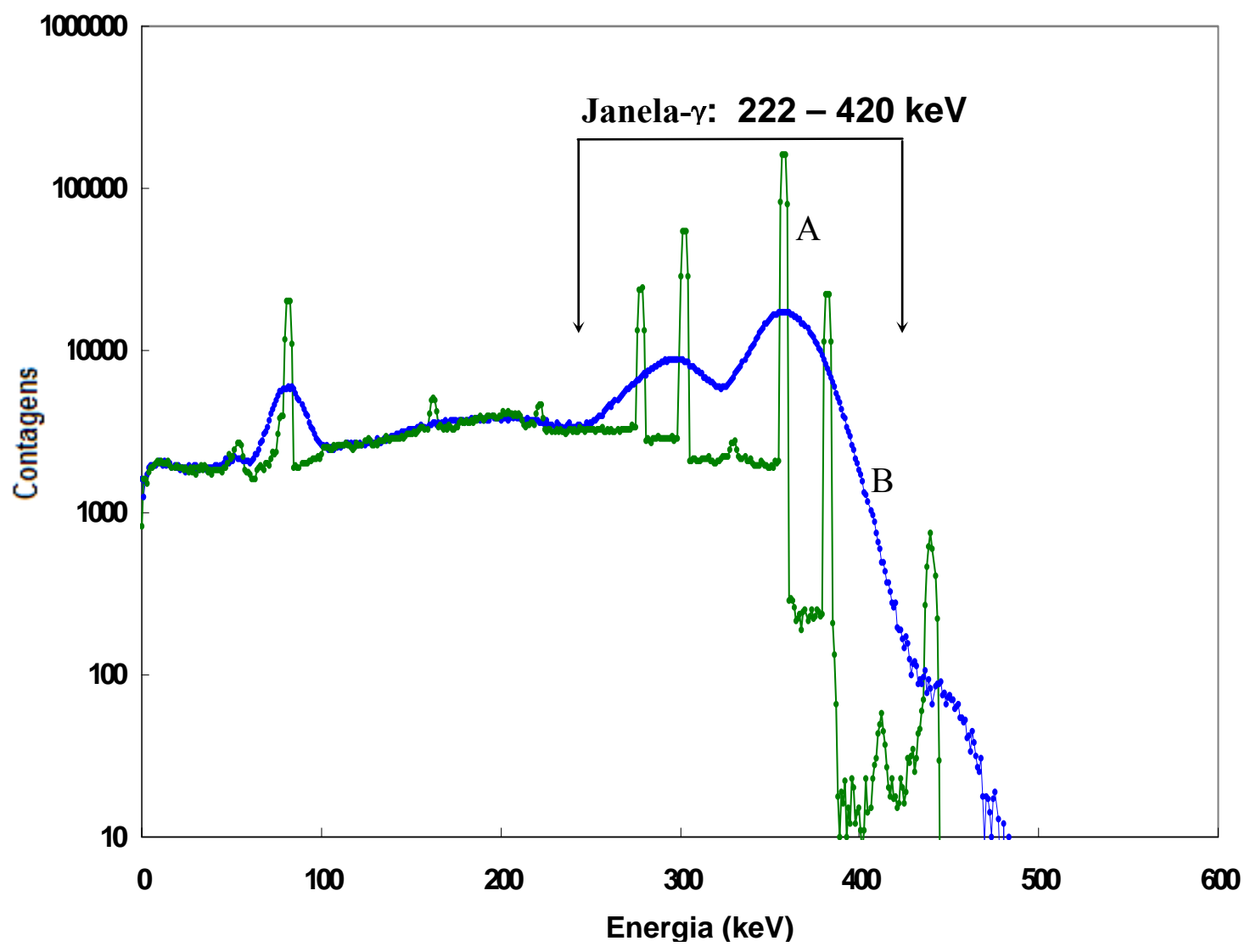

Figura 3.5 - Espectro gama simulado por Monte Carlo para o ${ }^{133} \mathrm{Ba}$. Espectro A não inclui efeito de resolução. Espectro $\mathrm{B}$ inclui efeito de resolução simulando o espectro experimental para energia de $661,6 \mathrm{keV}$.

A figura 3.6 mostra o comportamento da curva de $\frac{N_{\beta} N \gamma}{N_{C}}$ como função do parâmetro de ineficiência $\frac{1-\varepsilon_{\beta}}{\varepsilon_{\beta}}$. Os círculos vermelhos correspondem ao resultado experimental e os círculos na cor azul correspondem aos valores previstos pelo método de Monte Carlo, normalizado pelo valor da atividade. A linha cheia corresponde a um polinômio de segundo grau. Há uma boa concordância entre os pontos experimentais e os calculados por Monte Carlo. 


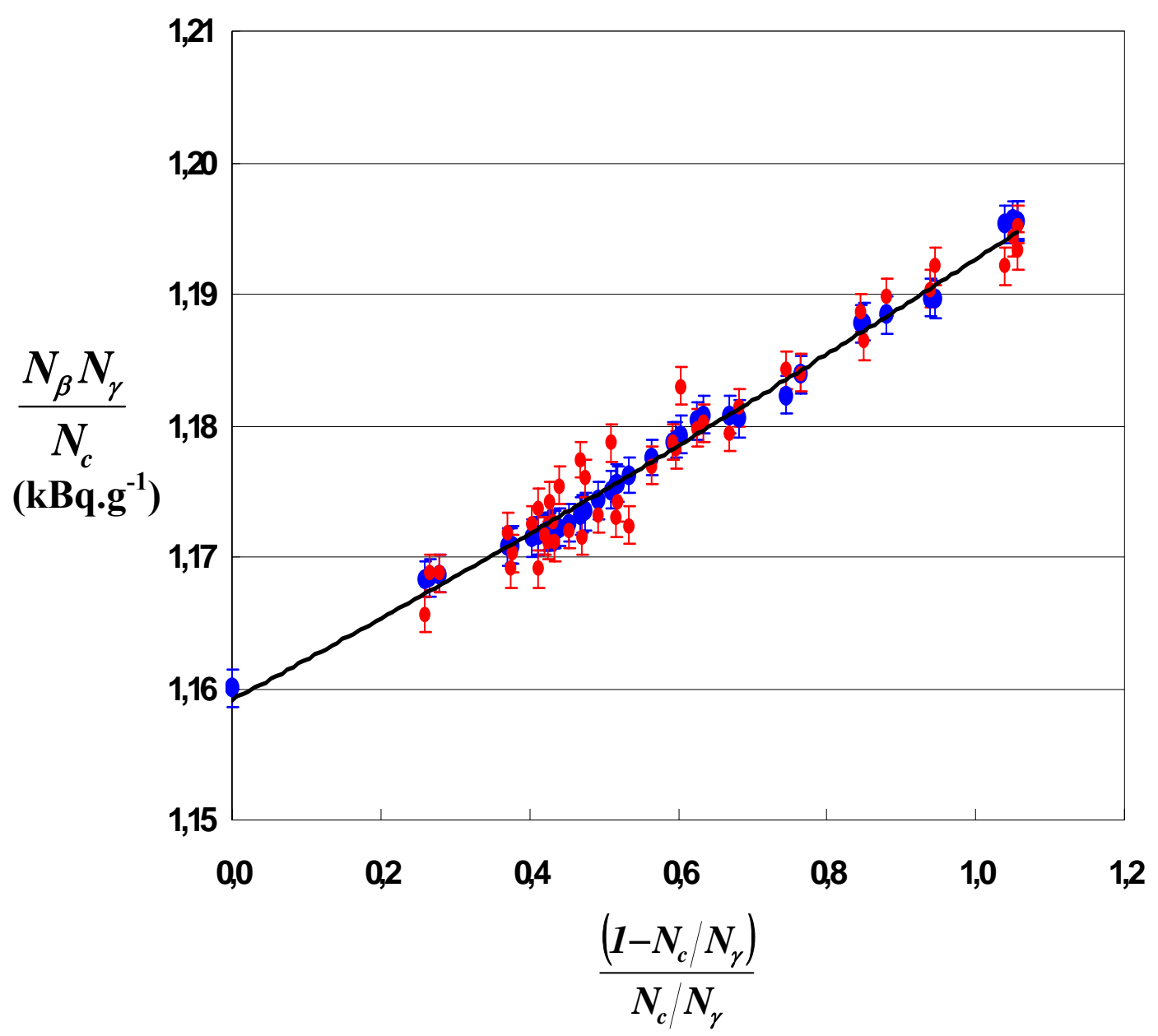

Figura 3.6 - Curvas dos resultados experimentais do ${ }^{133} \mathrm{Ba}$ comparadas com a simulação por Monte Carlo e polinômio de segundo grau.

A atividade obtida por intermédio do programa ESQUEMA foi de $1160,0(12) \mathrm{Bq}_{\mathrm{g}}{ }^{-1}$. Este valor está em excelente concordância com o valor de $1159,9(15) \mathrm{Bq}^{-g^{-1}}$ obtido utilizando o polinômio de segundo grau e com o valor médio obtido na comparação, 1160,8(42) Bq.g ${ }^{-1[52]}$.

Podemos concluir pelos resultados obtidos na padronização do ${ }^{133} \mathrm{Ba}$ (figura 3.6) que a simulação por Monte Carlo da curva de extrapolação está em boa concordância com a experimental para este radionuclídeo, que decai por Captura Eletrônica, apresentando uma pequena incerteza que é comparável à do ajuste polinomial. Entretanto, enquanto o ajuste polinomial não possui nenhuma base física, o método de Monte Carlo procura ser uma simulação, a mais próxima possível, do experimento efetuado. 


\subsection{Padronização do ${ }^{35} \mathrm{~S}$}

Fez-se uma simulação pelo método de Monte Carlo para a mistura de ${ }^{35} \mathrm{~S}+{ }^{60} \mathrm{Co}$ e foi efetuada uma comparação com o resultado experimental obtido no LMN. A janela gama utilizada para a discriminação gama abrange os picos de absorção total de $1173 \mathrm{keV}$ e $1332 \mathrm{keV}$, respectivamente.

A figura 3.7 mostra o comportamento da curva de $\frac{N_{\beta} N \gamma}{N_{C}}$ como função do parâmetro de ineficiência $\frac{1-\varepsilon_{\beta}}{\varepsilon_{\beta}}$. Os círculos cheios correspondem ao resultado experimental e os círculos vazios correspondem aos valores previstos pelo método de Monte Carlo. A linha tracejada corresponde ao ajuste por um polinômio de primeiro grau e a linha cheia a um polinômio de segundo grau.

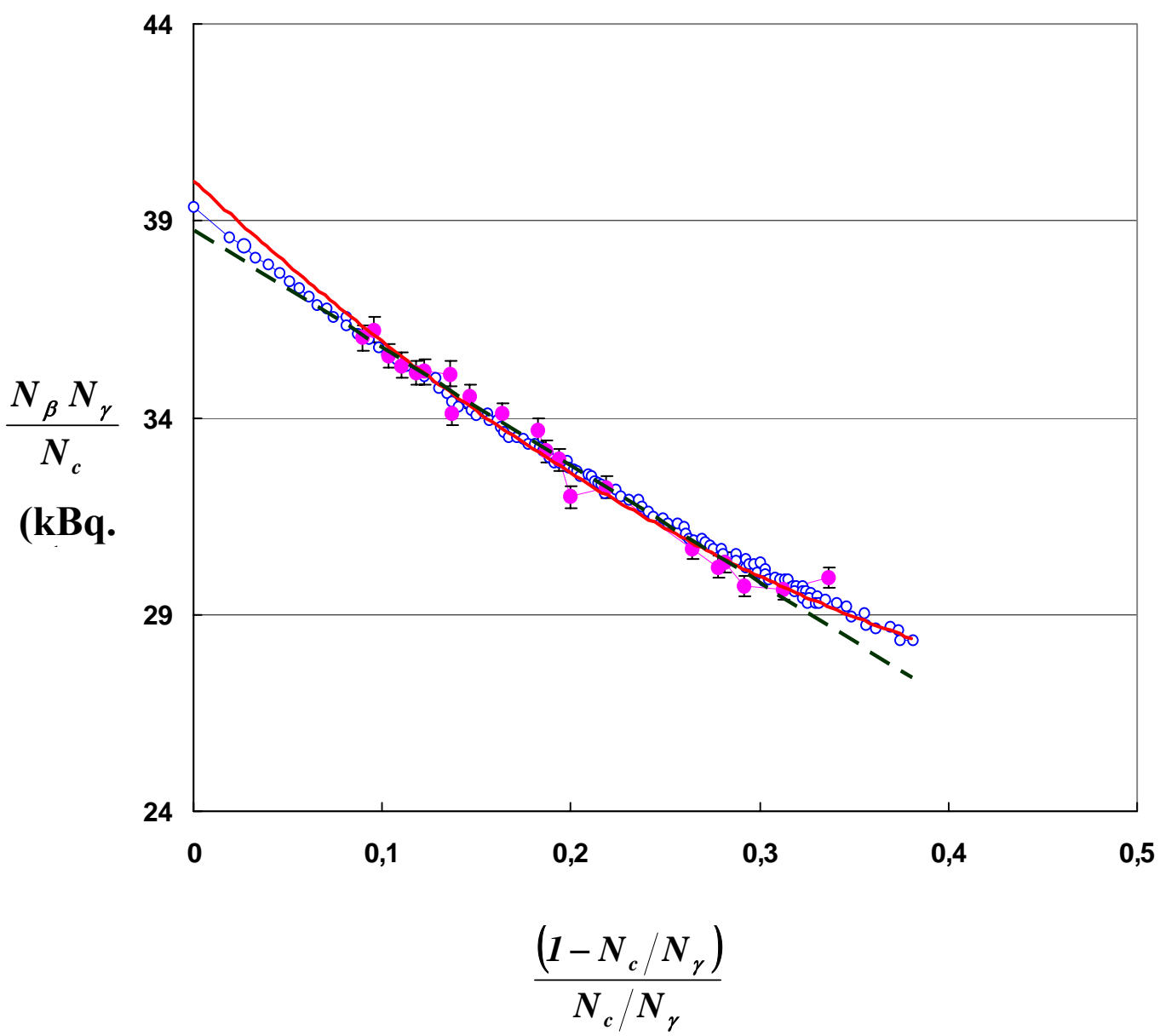

Figura 3.7 - Curvas dos resultados experimentais do ${ }^{35} \mathrm{~S}$ comparadas com a simulação por Monte Carlo e polinômios de primeiro e segundo graus. 
O resultado da atividade obtida pelo método dos mínimos quadrados de acordo com a equação 2.22 foi de $39,5(2) \mathrm{kBq} \cdot \mathrm{g}^{-1}$. De acordo com os polinômios de primeiro e segundo graus os resultados foram $38,8(3) \mathrm{kBq} \cdot \mathrm{g}^{-1}$ e $40,0(6) \mathrm{kBq} \cdot \mathrm{g}^{-1}$, respectivamente.

Conforme pode ser visto na figura 3.7, curva de extrapolação do ${ }^{35} \mathrm{~S}$, que é um emissor beta puro e foi utilizado o ${ }^{60} \mathrm{Co}$ como traçador, há uma boa concordância entre os pontos experimentais e os calculados por Monte Carlo dentro da incerteza estatística e indica um comportamento não linear. Os pontos obtidos por Monte Carlo situam-se entre as duas curvas polinomiais indicando que o procedimento por Monte Carlo sugere uma curva mais realista e pode simular satisfatoriamente as equações 2.11 e 2.17. 


\section{CONCLUSÃO}

Os resultados obtidos na tabela 2.5 indicam que, para essa geometria, os cálculos pelo método de Monte Carlo estão de acordo com os resultados analíticos, dentro da incerteza estatística.

Os resultados apresentados na tabela 2.6 mostram uma boa concordância entre os resultados obtidos por Monte Carlo e os dados experimentais indicando que a modelagem se aproxima do experimental.

Na Figura 2.23 as duas curvas estão de acordo na região de alta eficiência e o ajuste pelo método dos mínimos quadrados resultou na inclinação esperada $(0,0909)$ dentro da incerteza estatística para ambas as curvas. Para valores de abscissa acima de 1,0 (eficiência beta abaixo de 50\%), a curva B decresce, indicando que a eficiência do elétron de conversão está diminuindo para valores abaixo de 100\%.

Esses resultados indicam que o método de Monte Carlo permite um leque de alternativas na simulação aplicada à técnica da extrapolação da eficiência para determinar a atividade, tais como: variação na energia de corte para radiação beta depositada, que corresponde na prática a discriminação eletrônica; simulação de fonte sem absorvedor, que na prática não é possível obter; obter eficiência para beta próximas de $100 \%$, que na prática é difícil de obter, etc.

A simulação por Monte Carlo forneceu curvas de extrapolação em concordância com os pontos experimentais para os radionuclídeos estudados, que decaem por $\beta^{-}-\gamma\left({ }^{134} \mathrm{Cs} \mathrm{e}{ }^{72} \mathrm{Ga}\right)$ conforme item 3.1. e 3.2., Captura Eletrônica- $\gamma\left({ }^{133} \mathrm{Ba}\right)$ conforme item 3.3. e emissão $\beta^{-}$puro $\left({ }^{35} \mathrm{~S}+{ }^{60} \mathrm{Co}\right)$, este último padronizado pela Técnica do Traçador, conforme item 3.4. 
A aplicação do método de Extrapolação para a medida de atividade por coincidência $4 \pi \beta-\gamma$ coincide com as previsões por Monte Carlo para os casos estudados, com exceção do ${ }^{35} \mathrm{~S}$, cuja extrapolação convencional é incerta e ambígua. Neste caso, o resultado teórico pode ser considerado mais confiável, pois se baseia numa simulação próxima da realidade.

Os resultados obtidos nos casos mencionados anteriormente indicam a possibilidade da utilização do código desenvolvido no presente trabalho para qualquer radionuclídeo de interesse, cujo esquema de desintegração seja conhecido. Com base nas informações contidas no esquema de desintegração é possível desenvolver uma simulação que permite prever o espectro de coincidências, possibilitando a escolha da energia gama para o qual a atividade é obtida com melhor exatidão, incluindo a região em que a eficiência é próxima de $100 \%$.

A metodologia aplicando o método de Monte Carlo permite um planejamento adequado das medidas para o método de coincidências, principalmente para radionuclídeos com esquema de desintegração complexo.

A possibilidade de um planejamento para a escolha de energia gama que permite obter a atividade com melhor exatidão vem corroborar o excelente grau de confiança obtido nos sistemas de medidas desenvolvidos no IPEN, e um maior grau de exatidão na atividade obtida nas Comparações Internacionais patrocinadas pelo Bureau Internacional dès Poids et Mèsures, na França.

Este trabalho teve repercussão no $15^{\text {th }}$ International Conference on Radionuclide Metrology and its Applications - ICRM 2005, Oxford, England onde grupos de vários países, entre eles a Argentina, Korea, Itália, demonstraram interesse em conhecer o programa objeto do presente trabalho. A partir do convite do grupo da Argentina, o programa foi apresentado ao laboratório de metrologia do Centro Atómico Ezeiza - CNEA - Comision Nacional de Energia Atomica, Buenos Aires, Argentina, em março de 2006. 


\section{PERSPECTIVAS FUTURAS}

Está previsto, e já em fase de execução, um aprimoramento da modelagem da geometria do sistema $4 \pi(\mathrm{PC}) \beta-\gamma$, conforme apresentado na figura 5.1, para tornar o modelo mais realista. Neste novo modelo estão incluídos os materiais constituintes da blindagem e acessórios próximos aos detectores.

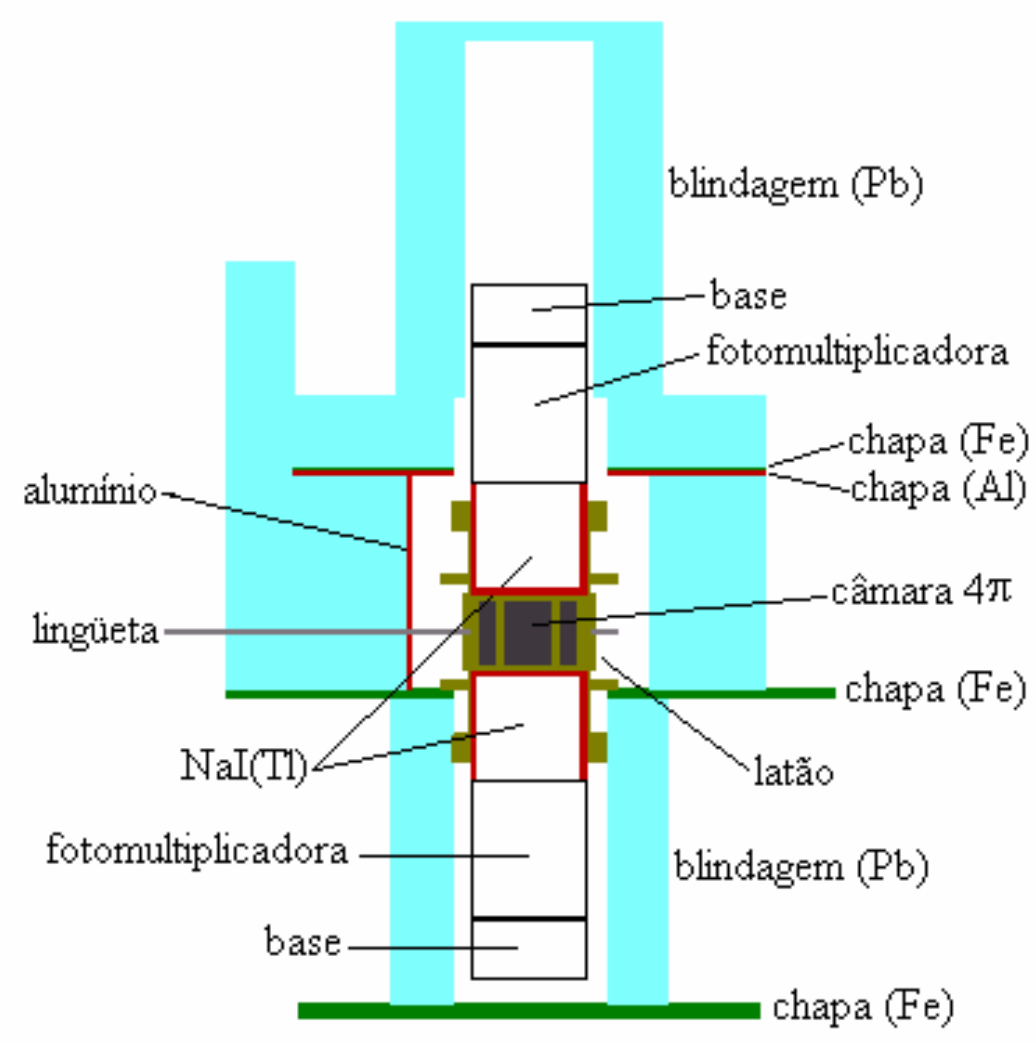

Figura 5.1 - Modelagem aprimorada da geometria do sistema $4 \pi \beta(\mathrm{PC})-\gamma$

Será acrescentada uma tabela com os valores da função de $\operatorname{Fermi~} F_{N}(Z, \eta)$ que estão tabuladas na referência ${ }^{[60]}$ para o espectro beta, considerando o efeito relativístico na equação 2.20. Pretende-se também efetuar uma estimativa da incerteza nos resultados do código ESQUEMA por meio de uma análise de sensibilidade, incorporando distribuições normais com desvio padrão pré-estabelecido para cada um dos parâmetros envolvidos no 
cálculo. Outra possibilidade é comparar os resultados do MCNP4C com outro código de transporte de radiação, e.g. PENELOPE ${ }^{[61]}$.

Estas melhorias no código estão previstas, com o objetivo de aperfeiçoar a modelagem, aproximando-se mais às condições experimentais e possibilitar a comparação dos resultados previstos por Monte Carlo, com curvas de extrapolação obtidas experimentalmente para outros radionuclídeos de esquema de desintegração complexos, ou que apresentem outros modos de desintegração, e.g. emissão $\beta^{+}$. Para a modelagem de emissores de pósitrons, os códigos indicados são PENELOPE ou MCNPX ${ }^{[62]}$. Com isto, haverá maior confiabilidade nos resultados simulados e permitirá que a presente metodologia torne-se uma ferramenta poderosa no planejamento de experimentos, utilizando a técnica de coincidências $4 \pi \beta-\gamma$. Este procedimento também evitará a ocorrência de ambigüidades na extrapolação, conforme pode-se constatar na literatura ${ }^{[63]}$. 


\section{APÊNDICE A - Código para Verificar o Período do Gerador de Números Aleatórios do Fortran}

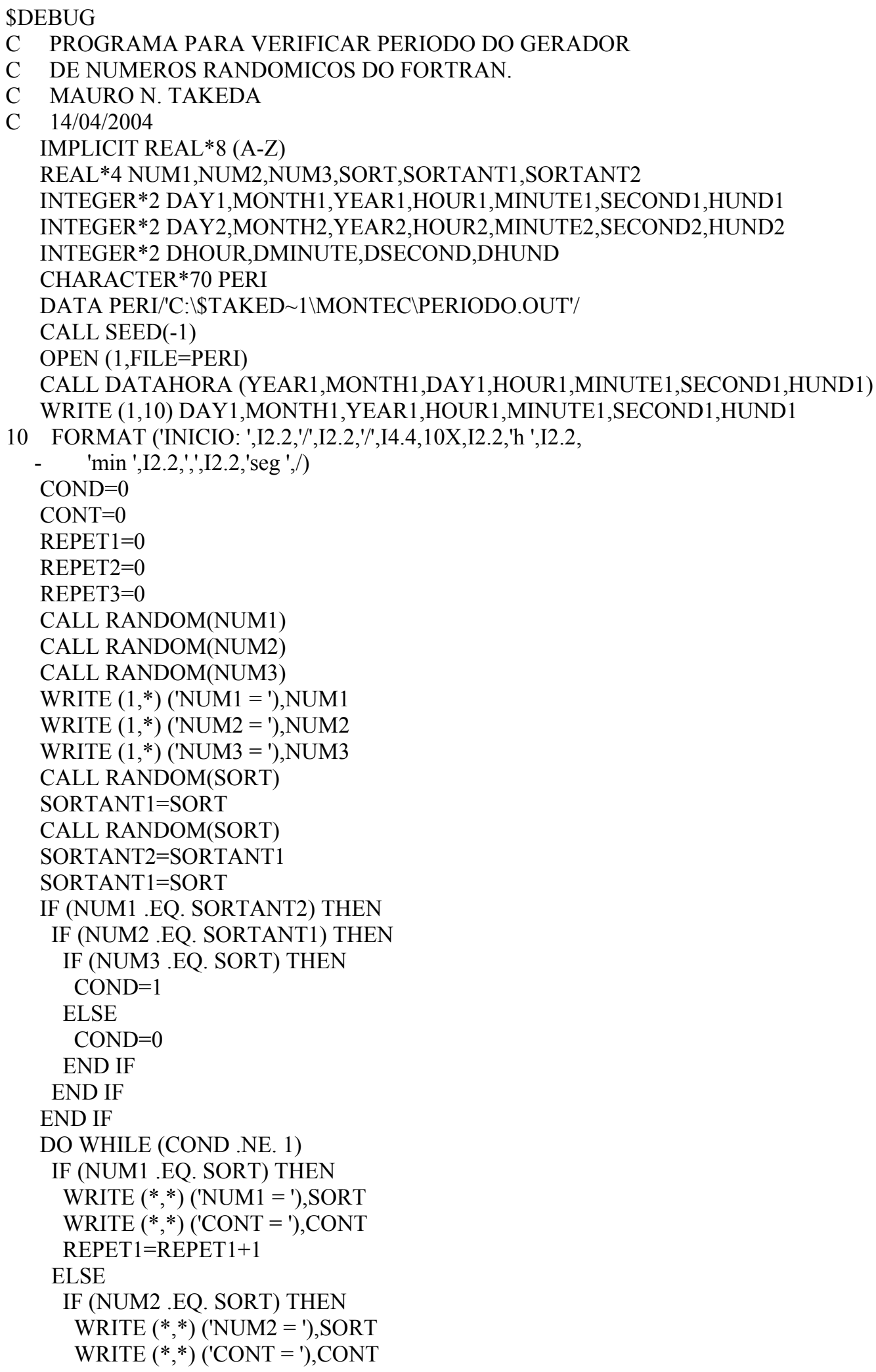




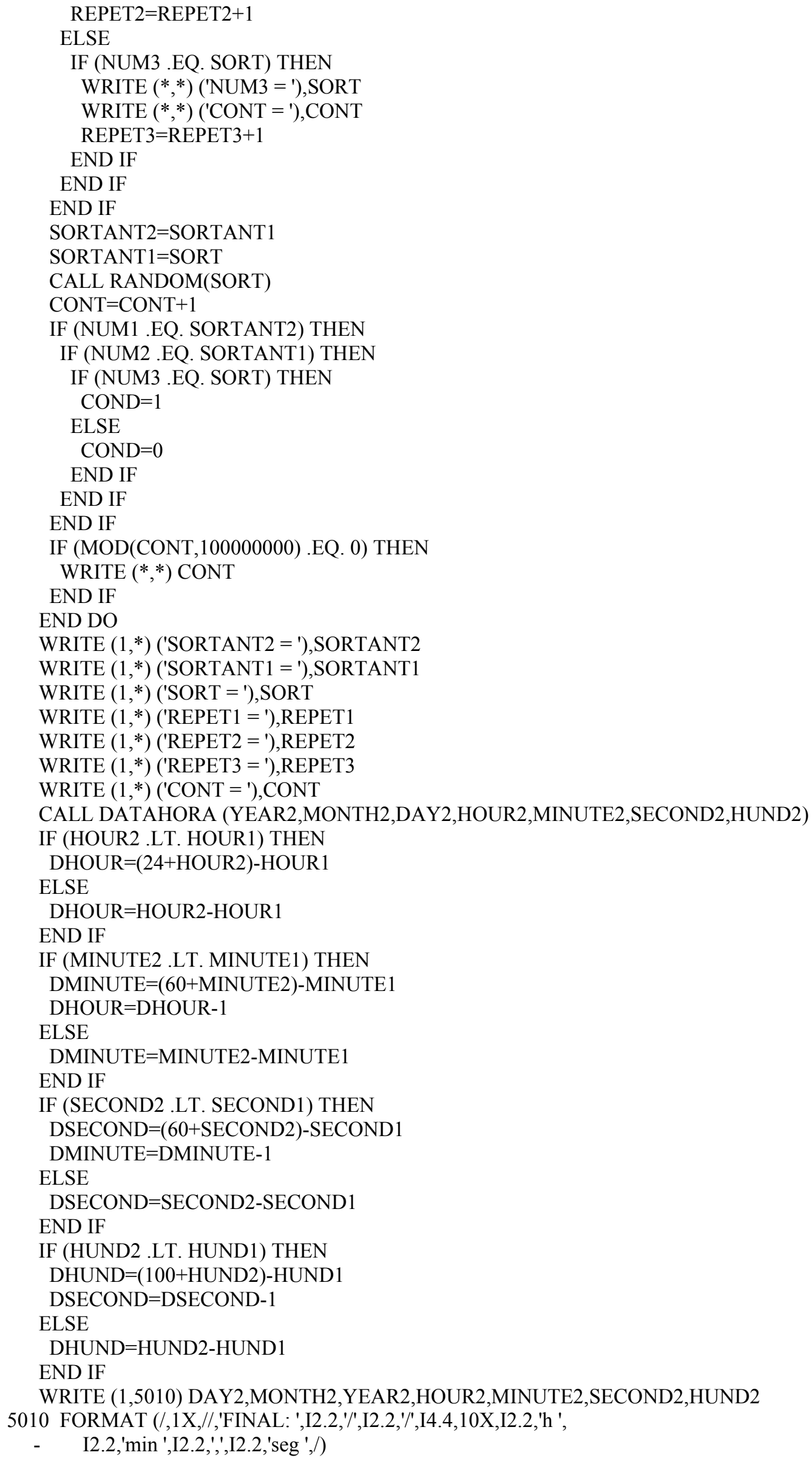


WRITE $(1,5020)$ DHOUR,DMINUTE,DSECOND,DHUND

5020 FORMAT ('TEMPO DE PROCESSAMENTO: ',I2.2,'h ',I2.2,'min ',I2.2,',',

I2.2,'seg ')

CLOSE (1)

STOP

END

C INICIO SUBROTINAS

SUBROUTINE DATAHORA (YEAR,MONTH,DAY,HOUR,MINUTE,SECOND,HUND)

INTEGER*2 YEAR,MONTH,DAY,HOUR,MINUTE,SECOND,HUND

CALL GETDAT(YEAR,MONTH,DAY)

CALL GETTIM(HOUR,MINUTE,SECOND,HUND)

RETURN

END 


\section{APÊNDICE B - Código para Verificar a Uniformidade na Geração de Números Aleatórios do Fortran}

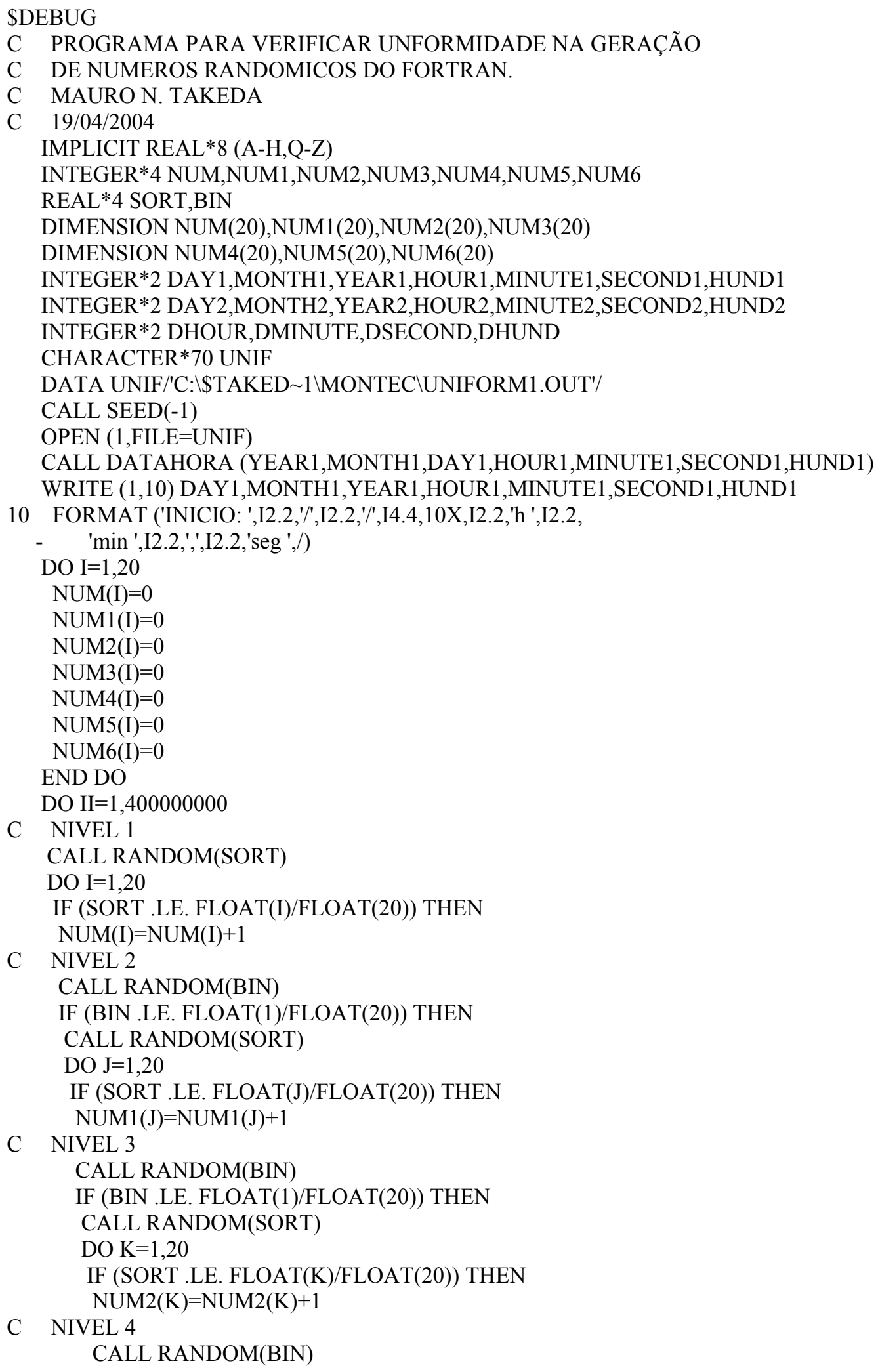

C NIVEL 2

CALL RANDOM(BIN)

IF (BIN .LE. FLOAT(1)/FLOAT(20)) THEN

CALL RANDOM(SORT)

DO J $=1,20$

IF (SORT .LE. FLOAT(J)/FLOAT(20)) THEN

C NIVEL 3$$
\operatorname{NUM} 1(\mathrm{~J})=\mathrm{NUM} 1(\mathrm{~J})+1
$$

CALL RANDOM(BIN)

IF (BIN .LE. FLOAT(1)/FLOAT(20)) THEN

CALL RANDOM(SORT)

DO $\mathrm{K}=1,20$

IF (SORT .LE. FLOAT(K)/FLOAT(20)) THEN

C NIVEL 4 $\mathrm{NUM} 2(\mathrm{~K})=\mathrm{NUM} 2(\mathrm{~K})+1$

CALL RANDOM(BIN) 


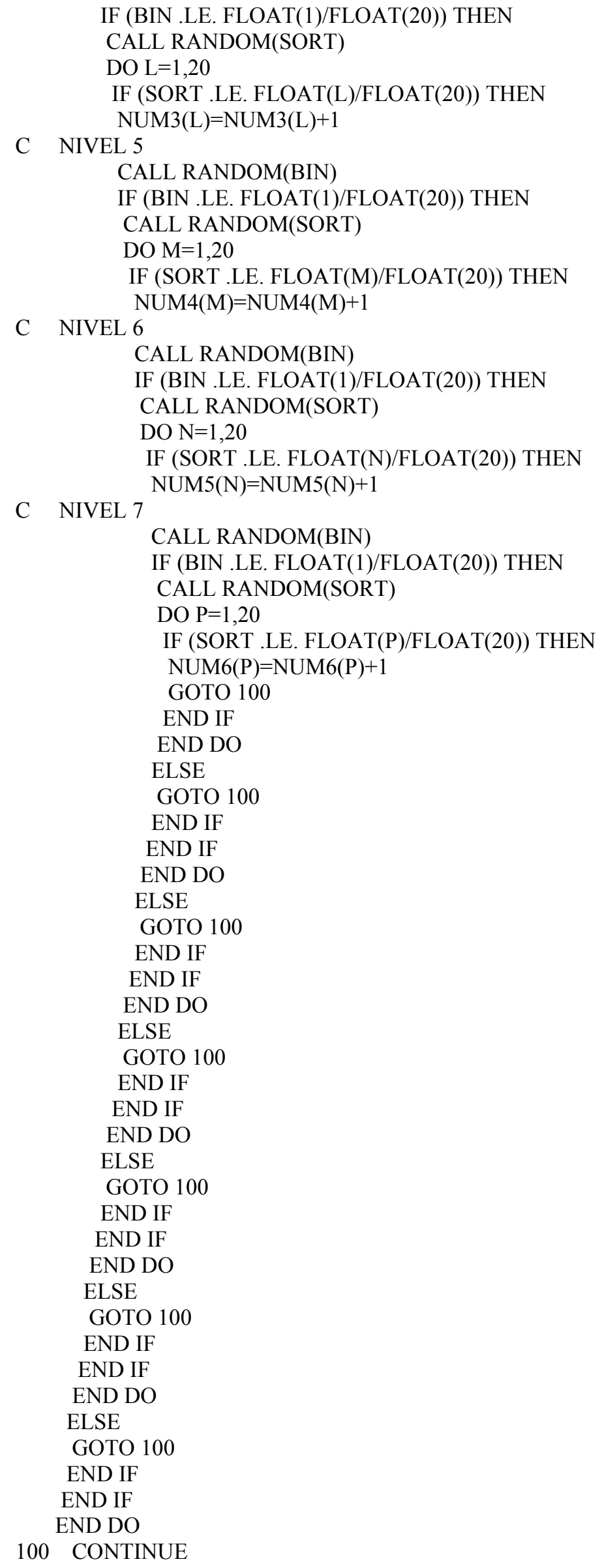


IF (MOD(II,10000000) .EQ. 0) THEN

$\operatorname{WRITE}(*, *)(' \mathrm{II}=$ '),II

END IF

END DO

WRITE $\left(1,{ }^{*}\right)($ 'CONTAGEM NO NÍVEL 1 ')

WRITE $(1,200)(\mathrm{NUM}(\mathrm{I}), \mathrm{I}=1,20)$

200 FORMAT (20I10)

WRITE $\left(1,{ }^{*}\right)($ 'CONTAGEM NO NÍVEL 2 ')

WRITE $(1,200)$ (NUM1(J), J=1,20)

WRITE $\left(1,{ }^{*}\right)($ 'CONTAGEM NO NÍVEL 3 ')

WRITE $(1,200)$ (NUM2(K), K=1,20)

WRITE $\left(1,{ }^{*}\right)($ 'CONTAGEM NO NÍVEL 4')

WRITE $(1,200)$ (NUM3(L), L=1,20)

WRITE $\left(1,{ }^{*}\right)($ 'CONTAGEM NO NÍVEL 5')

WRITE $(1,200)$ (NUM4(M), M=1,20)

WRITE $\left(1,{ }^{*}\right)($ 'CONTAGEM NO NÍVEL 6 ')

WRITE $(1,200)$ (NUM5(N), N=1,20)

WRITE $\left(1,{ }^{*}\right)($ 'CONTAGEM NO NÍVEL 7')

WRITE $(1,200)$ (NUM6(P), P=1,20)

CALL DATAHORA (YEAR2,MONTH2,DAY2,HOUR2,MINUTE2,SECOND2,HUND2)

IF (HOUR2 .LT. HOUR1) THEN

DHOUR $=(24+$ HOUR2 $)$ HOUR 1

ELSE

DHOUR=HOUR2-HOUR1

END IF

IF (MINUTE2 .LT. MINUTE1) THEN

DMINUTE $=(60+$ MINUTE2 $)$-MINUTE1

DHOUR=DHOUR -1

ELSE

DMINUTE=MINUTE2-MINUTE1

END IF

IF (SECOND2 .LT. SECOND1) THEN

DSECOND $=(60+$ SECOND2 $)$-SECOND1

DMINUTE=DMINUTE-1

ELSE

DSECOND=SECOND2-SECOND1

END IF

IF (HUND2 .LT. HUND1) THEN

DHUND $=(100+$ HUND2 $)$-HUND1

DSECOND=DSECOND-1

ELSE

DHUND=HUND2-HUND1

END IF

WRITE $(1,5010)$ DAY2,MONTH2,YEAR2,HOUR2,MINUTE2,SECOND2,HUND2

5010 FORMAT (/,1X,//,'FINAL: ',I2.2,,'/',I2.2,'/',I4.4,10X,I2.2,'h ',

- I2.2,'min ',I2.2,',',I2.2,'seg ',/)

WRITE $(1,5020)$ DHOUR,DMINUTE,DSECOND,DHUND

5020 FORMAT ('TEMPO DE PROCESSAMENTO: ',I2.2,'h ',I2.2,'min ',I2.2,',',

- $\quad$ I2.2,'seg ')

CLOSE (1)

STOP

END

C INICIO SUBROTINAS

SUBROUTINE DATAHORA (YEAR,MONTH,DAY,HOUR,MINUTE,SECOND,HUND)

INTEGER*2 YEAR,MONTH,DAY,HOUR,MINUTE,SECOND,HUND

CALL GETDAT(YEAR,MONTH,DAY)

CALL GETTIM(HOUR,MINUTE,SECOND,HUND)

RETURN

END 


\section{APÊNDICE C - Arquivo EFIBETA.DAT}

Vetor de energias para elétrons $(\mathrm{MeV})$.

0.0000000 .0000100 .0000200 .0000300 .0000400 .0000500 .0000600 .000070 0.0000800 .0000900 .0001000 .0001100 .0001200 .0001300 .0001400 .000150

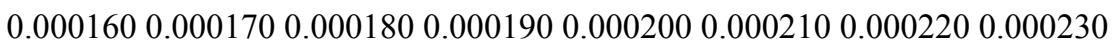

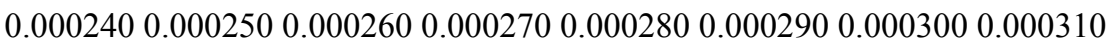

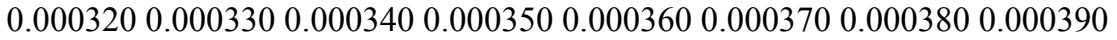

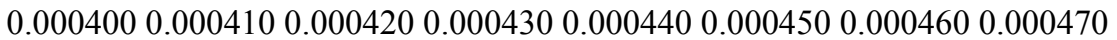

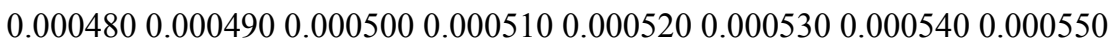
0.0005600 .0005700 .0005800 .0005900 .0006000 .0006100 .0006200 .000630 0.0006400 .0006500 .0006600 .0006700 .0006800 .0006900 .0007000 .000710

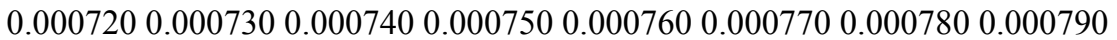

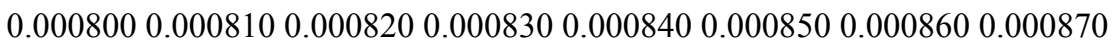

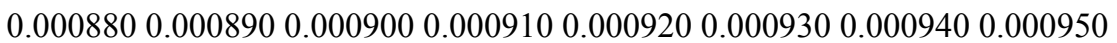
0.0009600 .0009700 .0009800 .0009900 .0010000 .0012000 .0014000 .001600 0.0018000 .0020000 .0022000 .0024000 .0026000 .0028000 .0030000 .003200

3.1500003 .2000003 .2500003 .3000003 .3500003 .4000003 .4500003 .500000 3.5500003 .6000003 .6500003 .7000003 .7500003 .8000003 .8500003 .900000 3.9500004 .000000

Vetor das eficiências dos elétrons monoenergéticos no $4 \pi$ normalizada e integrada, a $1^{\mathrm{a}}$ coluna corresponde a energia de $0,0014 \mathrm{MeV}$, a $2^{\mathrm{a}}$ coluna a energia de $0,0016 \mathrm{MeV}$ e assim sucessivamente, as linhas correspondem as eficiências para as energias do intervalo de 0 a energia considerada.

0.0000000 .0000000 .0000000 .0000000 .0000000 .0000000 .0000000 .000000 0.0029950 .0025000 .0021020 .0016500 .0012770 .0009900 .0007640 .000617 0.0062060 .0052670 .0044250 .0034790 .0027050 .0020680 .0016160 .001301 0.0094740 .0082030 .0069690 .0055100 .0042770 .0032670 .0025590 .002064 0.0126420 .0111900 .0096160 .0076610 .0059580 .0045540 .0035690 .002883 0.0156110 .0141290 .0123080 .0098630 .0077070 .0059100 .0046390 .003743 0.0182940 .0169550 .0149270 .0120760 .0094920 .0072970 .0057320 .004619 0.0206600 .0196340 .0174830 .0142890 .0112720 .0087010 .0068330 .005512 0.0227510 .0221540 .0199740 .0164690 .0130490 .0101080 .0079570 .006413 0.0246120 .0245880 .0224320 .0186280 .0148330 .0115140 .0090770 .007324

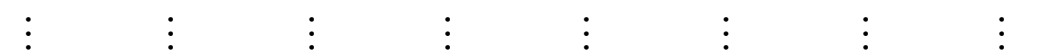

0.0396230 .0610220 .0837820 .1056830 .1175620 .1176800 .1084140 .095915 0.8275800 .4963880 .2958440 .2096470 .1817200 .1668190 .1516220 .134909 1.0000000 .9040840 .6303980 .3813150 .2785190 .2307120 .2012840 .177872 0.0000001 .0000000 .9129580 .6230070 .4147870 .3140530 .2595750 .224396 0.0000000 .0000001 .0000000 .8957670 .6411840 .4469990 .3446610 .285941 0.0000000 .0000000 .0000001 .0000000 .8888440 .6454680 .4653440 .366776 0.0000000 .0000000 .0000000 .0000001 .0000000 .8785400 .6472790 .481661 0.0000000 .0000000 .0000000 .0000000 .0000001 .0000000 .8722050 .659201 0.0000000 .0000000 .0000000 .0000000 .0000000 .0000001 .0000000 .882123 0.0000000 .0000000 .0000000 .0000000 .0000000 .0000000 .0000001 .000000 


\section{APÊNDICE D - Arquivo EFIGAMA.DAT}

Vetor das eficiências para gama no $\mathrm{NaI}(\mathrm{Tl})$ normalizada e integrada, a $1^{\text {a }}$ coluna corresponde a energia de $0,048 \mathrm{MeV}$, a $2^{\mathrm{a}}$ coluna a energia de $0,052 \mathrm{MeV}$ e assim sucessivamente, as linhas correspondem as eficiências para as energias do intervalo de 0 a energia considerada.

0.0000000 .0000000 .0000000 .0000000 .0000000 .0000000 .0000000 .000000 0.0000000 .0001110 .0000300 .0000800 .0000530 .0000490 .0000530 .000062 0.0000000 .0005570 .0006400 .0005230 .0004130 .0003060 .0002340 .000223 0.0000000 .0011150 .0021950 .0024460 .0019630 .0014330 .0010530 .000853 0.0346600 .0022300 .0023780 .0026170 .0027250 .0030020 .0034540 .002770 0.1566110 .0311040 .0034440 .0029690 .0029410 .0031780 .0035740 .003971 0.1578950 .1376810 .0303590 .0043570 .0034650 .0034610 .0037960 .004149 0.1578950 .1381270 .1262540 .0303650 .0050790 .0041040 .0041070 .004402 0.1578950 .1381270 .1266800 .1144290 .0286370 .0061230 .0049560 .004854 0.1578950 .1383500 .1266800 .1150320 .1027390 .0275910 .0072800 .005917 0.1591780 .1386850 .1268630 .1151120 .1033310 .0935550 .0268290 .008496 0.1643130 .1402450 .1276860 .1154650 .1035170 .0941520 .0856440 .026447 1.0000000 .1464880 .1292410 .1165680 .1042680 .0946770 .0863520 .078579 0.0000001 .0000000 .1398480 .1184800 .1055800 .0955940 .0871220 .079598 0.0000000 .0000001 .0000000 .1331670 .1090500 .0973390 .0882440 .080498 0.0000000 .0000000 .0000001 .0000000 .1280850 .1029190 .0908780 .082222 0.0000000 .0000000 .0000000 .0000001 .0000000 .1262620 .0989280 .085832 0.0000000 .0000000 .0000000 .0000000 .0000001 .0000000 .1268550 .096872 0.0000000 .0000000 .0000000 .0000000 .0000000 .0000001 .0000000 .129017 0.0000000 .0000000 .0000000 .0000000 .0000000 .0000000 .0000001 .000000

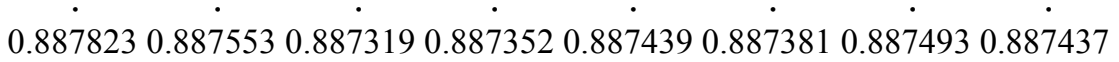
$\begin{array}{llllllll}0.887918 & 0.888020 & 0.887615 & 0.887411 & 0.887575 & 0.887435 & 0.887581 & 0.887544\end{array}$

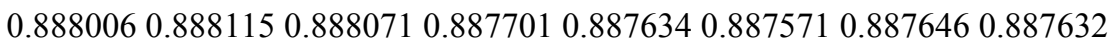
0.8881130 .8882150 .8881590 .8881800 .8879240 .8876420 .8877770 .887697 $\begin{array}{llllllll}0.888184 & 0.888328 & 0.888266 & 0.888269 & 0.888420 & 0.887931 & 0.887848 & 0.887822\end{array}$ 0.8882370 .8883990 .8883780 .8883750 .8885090 .8884340 .8881310 .887898 0.8882550 .8884520 .8884490 .8884870 .8886100 .8885290 .8886340 .888170 1.0000000 .8884870 .8885020 .8885580 .8887220 .8886120 .8887290 .888667 0.0000001 .0000000 .8885440 .8886120 .8887930 .8887180 .8888050 .888761

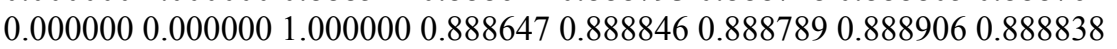
0.0000000 .0000000 .0000001 .0000000 .8888760 .8888420 .8889830 .888939 0.0000000 .0000000 .0000000 .0000001 .0000000 .8888780 .8890360 .889004 0.0000000 .0000000 .0000000 .0000000 .0000001 .0000000 .8890830 .889063 0.0000000 .0000000 .0000000 .0000000 .0000000 .0000001 .0000000 .889104 0.0000000 .0000000 .0000000 .0000000 .0000000 .0000000 .0000001 .000000

Vetor de energias para gama $(\mathrm{MeV})$.

0.0000000 .0040040 .0080040 .0120040 .0160040 .0200040 .0240040 .028004 0.0320040 .0360040 .0400040 .0440040 .0480040 .0520040 .0560040 .060004 0.0640040 .0680040 .0720040 .0760040 .0800040 .0840040 .0880040 .092004 0.0960040 .1000000 .1040000 .1080000 .1120000 .1160000 .1200000 .124000 $\begin{array}{lllllllllllllll}0.128000 & 0.132000 & 0.136000 & 0.140000 & 0.144000 & 0.148000 & 0.152000 & 0.156000\end{array}$

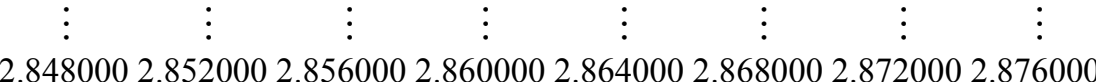
2.8800002 .8840002 .8880002 .8920002 .8960002 .9000002 .9040002 .908000 2.9120002 .9160002 .9200002 .9240002 .9280002 .9320002 .9360002 .940000 2.9440002 .9480002 .9520002 .9560002 .9600002 .9640002 .9680002 .972000 2.9760002 .9800002 .9840002 .9880002 .9920002 .9960003 .0000003 .004000 


\section{APÊNDICE E - Arquivo EFIGAMAT.DAT}

Vetor de eficiência total para gama, na primeira linha o $1^{\circ}$ valor corresponde a energia de $0,048 \mathrm{MeV}$, o $2^{\circ}$ valor $0,052 \mathrm{MeV}$ e assim sucessivamente. 0.0000310 .0001790 .0006560 .0017580 .0037800 .0069350 .0113030 .016857 $\begin{array}{llllllll}0.023412 & 0.030804 & 0.038829 & 0.047320 & 0.056063 & 0.064951 & 0.074021 & 0.082818\end{array}$ 0.0915390 .0999610 .1081130 .1160440 .1236260 .1309060 .1378060 .144425 0.1506270 .1566010 .1621900 .1675250 .1725320 .1772570 .1816960 .185903 0.1897690 .1935010 .1969280 .2001570 .2031660 .2059950 .2086450 .211119

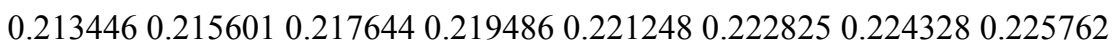

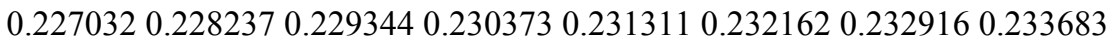
$\begin{array}{lllllllll}0.234343 & 0.234946 & 0.235460 & 0.235952 & 0.236441 & 0.236846 & 0.237208 & 0.237522\end{array}$

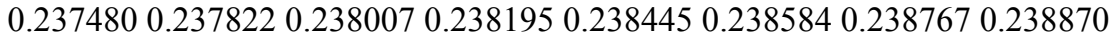

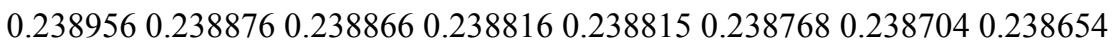

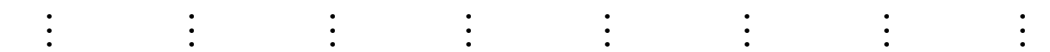

0.1676860 .1677350 .1677340 .1677800 .1677360 .1677630 .1677870 .167748 0.1677850 .1677800 .1677810 .1678050 .1678590 .1679210 .1679380 .167939 0.1679550 .1679680 .1680190 .1680620 .1681060 .1680640 .1680490 .168061 0.1681080 .1681370 .1681100 .1681420 .1681720 .1681840 .1682270 .168235 0.1681880 .1682120 .1682120 .1681910 .1682100 .1682800 .1682940 .168341 0.1683980 .1684760 .1684900 .1685330 .1685570 .1685660 .1685920 .168638 0.1686470 .1686720 .1686780 .1686900 .1687210 .1687550 .1687880 .168765 0.1687580 .1687670 .1687820 .1687910 .1688160 .1688040 .1687650 .168764 0.1687660 .1687880 .1688480 .1688610 .1688420 .1688970 .1688790 .168964 0.1689830 .1690300 .1690620 .1690750 .1690990 .1690840 .1691180 .169168 0.1692020 .1692090 .1692290 .169293 


\section{APÊNDICE F - Arquivo CONSTANT.DAT}

Disposição dos dados de entrada do arquivo CONSTANT.DAT.

DISCE DISCD FEMF FEMB FEMG COELIN NCT NHIST

XCOL1 XCOL2 NCOL ERESOL RESOL TRAC EBmin RARAN

NA QUAL:

DISCE - energia de corte dos elétrons emitidos $(\mathrm{MeV})$

DISCD - energia de corte dos elétrons depositados no detector $(\mathrm{MeV})$

FEMF - fator de conversão de energia máxima do elétron emitido em canal

FEMB - fator de conversão de energia máxima do elétron depositado em canal

$$
\mathrm{FEMF}=\mathrm{FEMB}<\text { int eiro }\left(\frac{\text { número de canais }}{\text { maior energia do elétron }[\mathrm{MeV}]}\right)
$$

FEMG - fator de conversão de energia depositada gama em canal

$$
\text { FEMG }<\text { int eiro }\left(\frac{\text { número de canais }}{\text { maior energia gama }[\mathrm{MeV}]}\right)
$$

COELIN - coeficiente linear para calibração em canal X energia gama

NCT - número de canais total

NHIST - número de histórias

XCOL1 - espessura mínima do absorvedor $\left(\mathrm{mg} / \mathrm{cm}^{2}\right)$

XCOL2 - espessura máxima do absorvedor $\left(\mathrm{mg} / \mathrm{cm}^{2}\right)$

NCOL - número de intervalos para absorvedor

ERESOL - energia para calibração do detector gama em resolução

RESOL - resolução relativa $\left(\mathrm{FWHM} / \mathrm{H}_{0}\right)$

TRAC - "flag" para traçador (beta-gama ou CE-gama $=0$, e traçador $=1$ )

EBmin - energia beta mínima da tabela de resposta

RARAN - raio da arandela $\left(\mathrm{mg} / \mathrm{cm}^{2}\right)$

Exemplo de arquivo CONSTANT.DAT para o ${ }^{134} \mathrm{Cs}$, com efeito de resolução.

$\begin{array}{lllllllll}0 & 000030 & 100 & 100 & 750 & 0 & 1024 & 1000000\end{array}$

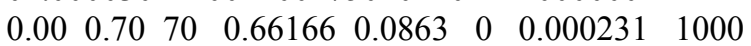




\section{APÊNDICE G - Arquivo ESQUEMA.DAT}

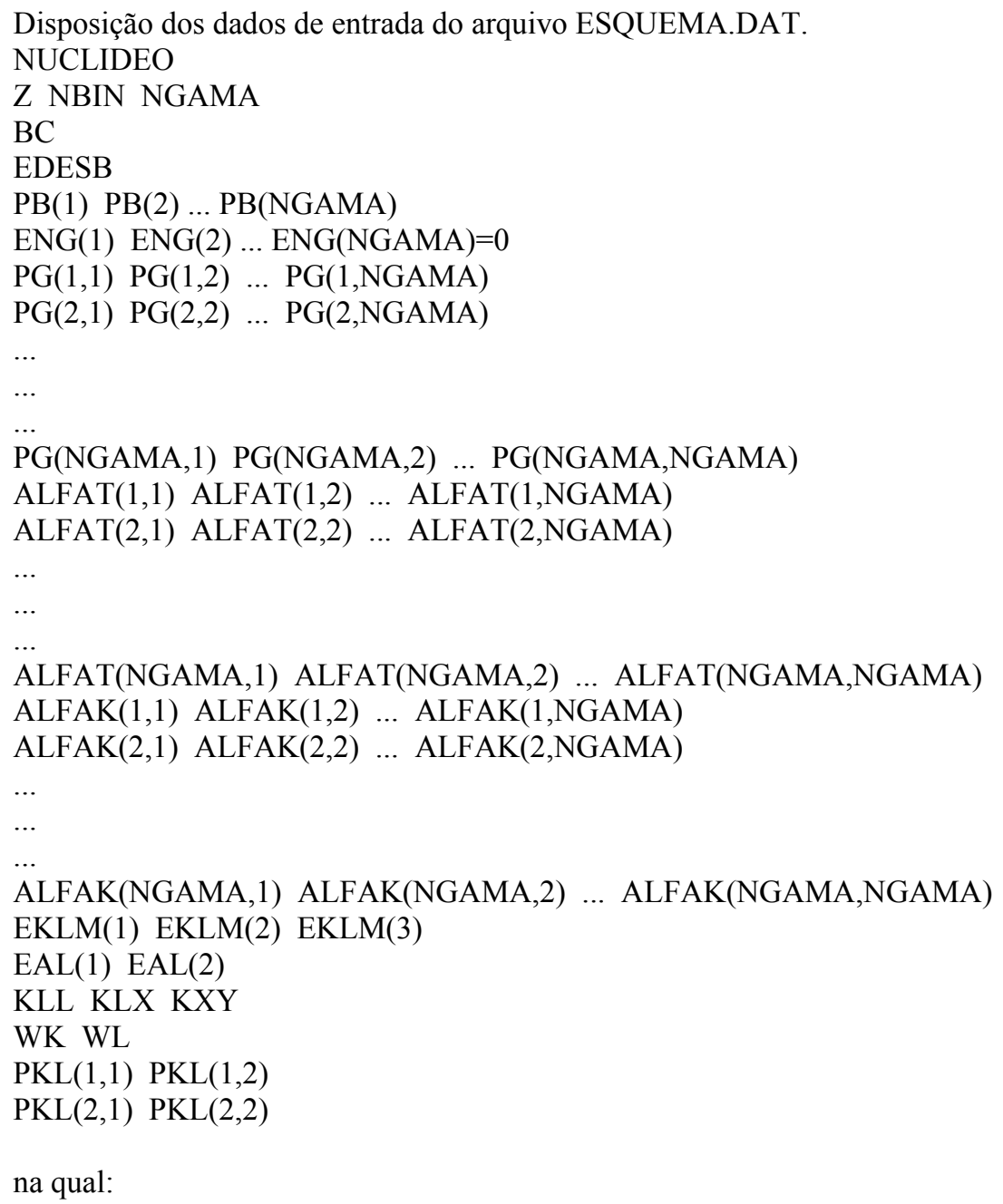

$\operatorname{ALFAK}(\mathrm{I}, \mathrm{J})$ - matriz dos coeficientes de conversão interna da camada $\mathrm{K}$, valores do esquema sem normalizar $(\mathrm{I}=1,2, \ldots$, NGAMA e $\mathrm{J}=1,2, \ldots, \mathrm{NGAMA})$

$\operatorname{EKLM}(1), \operatorname{EKLM}(2), \operatorname{EKLM}(3)$ - energia de ligação do elétron na camada K, L e M, respectivamente, do núcleo filho, em MeV

EAL(1), EAL(2) - energia mínima e máxima, respectivamente, do elétron Auger da camada L, em MeV

KLL, KLX e KXY - intensidade relativa do elétron Auger na camada K

WK, WL - probabilidades de emissão raio X nas camadas K e L 
PKL(I, J) - probabilidade de ocorrer a captura na camada $\mathrm{K}$ ou L no i-ésimo ramo captura, normalizado $=1$ por linha, $(\mathrm{I}=1,2) . \mathrm{J}=1 \rightarrow$ camada $\mathrm{K}, \mathrm{J}=2 \rightarrow$ camada $\mathrm{L}$.

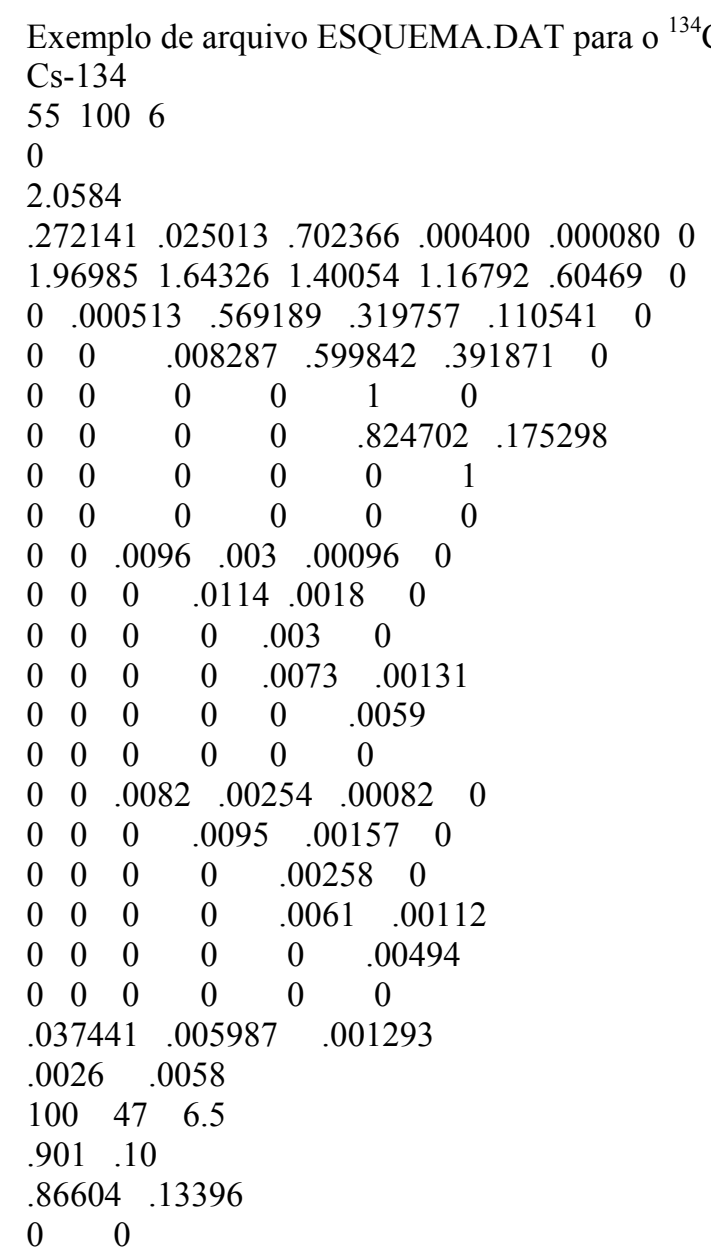




\section{APÊNDICE H - Arquivo NPICOS.DAT}

Disposição dos dados de entrada do arquivo NPICOS.DAT.

NPIC

CANAL1 CANAL2

$\cdots$

CANAL1 CANAL2

na qual:

NPIC - quantidade de intervalos gama em canais CANAL1 - canal inicial do intervalo de interesse CANAL2 - canal final do intervalo de interesse

Exemplo de arquivo NPICOS.DAT para o ${ }^{134} \mathrm{Cs}$.

2

390510

550650 


\section{APÊNDICE I - Arquivo FERMI.DAT}

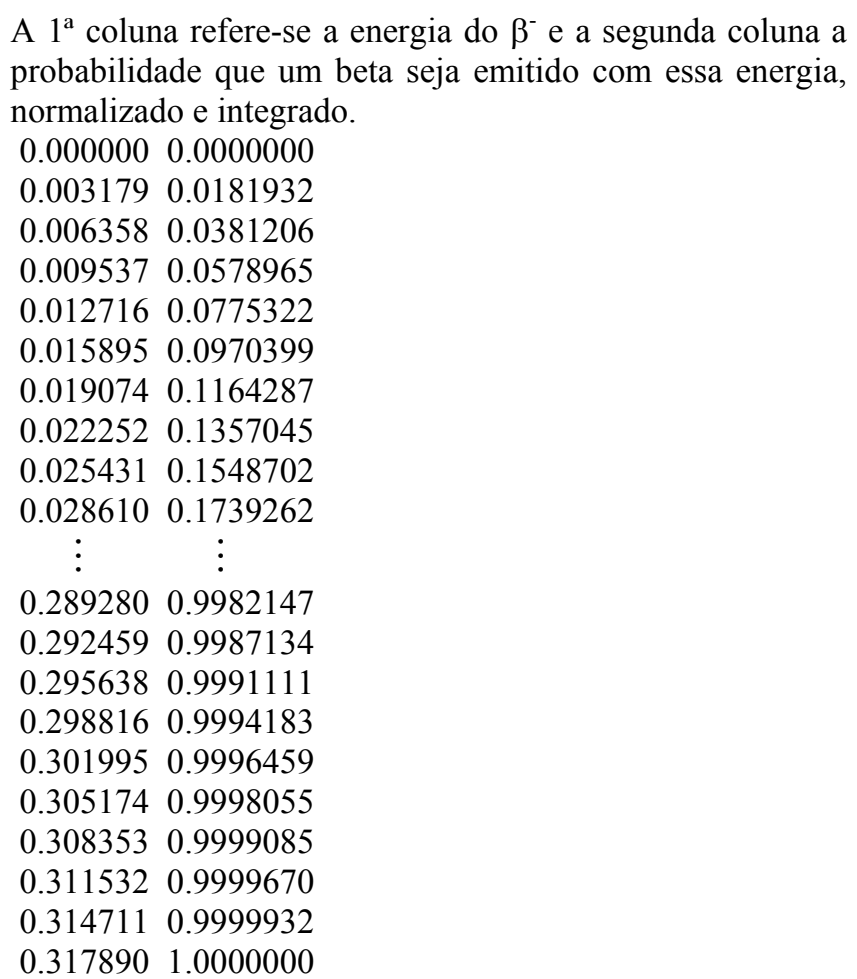




\title{
APÊNDICE J - Código do Programa ESQUEMA
}

\begin{abstract}
Abaixo é feito uma descrição dos procedimentos utilizados no programa ESQUEMA, as variáveis e os arquivos de entrada utilizados que constam dos apêndices C, D, E, F e H. A versão executável do programa está dispoível no LMN do IPEN-CNEN/SP.
\end{abstract}

\$DEBUG

C CALCULA NB*NG/NC E (1-NC/NG)/(NC/NG) PARA O SISTEMA 4PI-BETAGAMA (NaI(Tl))

C MODIFICAÇÃO DO PROGRAMA ESQUEMAM.FOR PARA CAPTURA ELETRONICA.

$\mathrm{C}$ INCLUSAO DA ENERGIA MINIMA PARA TABELA EFICIENCIA BETA

C CALCULA TABELA FERMI E IMPLEMENTADO N RAMOS

C BETA, GAMA COM 750 CANAIS VARIANDO DE $4 \mathrm{MeV}$ e

C GAMA EM CASCATA CONSIDERANDO O EFEITO SOMA.

C CALIBRACAO EM CANAL X ENERGIA

C FATOR DE GANHO PARA BETA DIFERENTE DO FATOR DE GANHO PARA FOTON

C CALCULA ENERGIA DOS ELETRONS DE CONVERSAO PARA AS CAMADAS K E L

C POR DIFERENCA ENTRE ENERGIA DO GAMA E ENERGIA DE LIGACAO DO ELETRON

C NA CAMADA CORRESPONDENTE.

C SEGUE ESQUEMA DE DESINTEGRAÇÃO PARA GAMA E ec ATÉ O ESTADO FUNDAMENTAL

C GERA NOVA SEMENTE PARA NUMEROS ALEATORIOS QUANDO TOTAL DE NUMEROS

C ALEATORIOS ESTA PROXIMO DO PERIODO $(2,147 \mathrm{E}+9)$

C V 1.0 - FORTRAN Visual Workbench v 1.00

C 19/03/2006 - Mauro N. Takeda

C CALCULA ESPECTROS BETA, GAMA E COINCIDENCIA

C IMPLEMENTADO RESOLUCAO DO DETECTOR

C VARIAVEIS:

C $\quad$ FOT $=$ FATOR DE OTIMIZACAO $($ FOT $*$ EFICIENCIA $<100 \%)$

C DISCE $=$ ENERGIA DO DISCRIMINADOR PARA ENERGIA EMITIDA [MeV]

C DISCD $=$ ENERGIA DO DISCRIMINADOR PARA ENERGIA DEPOSITADA [MeV]

C $\quad$ FEMB $=$ FATOR DE ENERGIA MAXIMA PARA BETA

C $\quad F E M G=$ FATOR DE ENERGIA MAXIMA PARA FOTON

C NCT $=$ NUMERO DE CANAIS TOTAL

C LINCOL $=$ NUMERO DE LINHAS E COLUNAS

C NHIST $=$ NUMERO DE HISTORIAS

C $\quad$ FLAGcol $=$ FLAG PARA ABSORVEDOR DE COLLODION $(0=\mathrm{SEM}, 1=\mathrm{COM})$

C FLAGal $=$ FLAG PARA ABSORVEDOR DE ALUMINIO $(0=\mathrm{SEM}, 1=\mathrm{COM})$

C Xcol = ESPESSURA DO COLLODION $[\mathrm{mg} / \mathrm{cm} 2]$

C Xal $=$ ESPESSURA DO ALUMINIO $[\mathrm{mg} / \mathrm{cm} 2]$

IMPLICIT REAL*4 (A-H,O-Z)

INTEGER *4 CANALFE,CNLSBe,CANALEBD,CANALB,CANALG,CANALC,TRAC,SNUC

DIMENSION EB0(30),PB(30),EBI(78),ENG(30),EFGT(800),BETAi(30)

DIMENSION ERANGE(43),RANGECol(43),RANGEAl(43), EG(752), EKLM(3)

DIMENSION CANALB(4096),CANALG(4096),CANALC(4096),CANALEBD(4096)

DIMENSION CANALFE(4096),CNLSBe(4096),EeL(30,30),GAMAi(30,30)

DIMENSION PG(30,30),ALFA(30,30),EFG(800,800),EeK(30,30),eci(30,30)

DIMENSION EFELM(78,78),ALFAK(30,30),ALFAL(30,30),TOTGMi(30)

DIMENSION TOTeci(30),NEVGi(30,30),CAPi(30),PCAP(30),NEVKLi(30,2)

DIMENSION PGC $(30,30)$

REAL*4 EQ1(1001),EQ2(1001),EQ3(1001),EQ4(1001),EQ5(1001)

REAL*4 EQ6(1001),EQ7(1001),EQ8(1001),EQ11(1001),EAL(2),PKL(2,2)

REAL*4 NNFERMI(1001,60),EBFERMI(1001,60),Xcol,Xal,ENGC(30)

INTEGER $* 2$ FOT,NCT,Z,NBIN

INTEGER*2 FLAGcol,FLAGal,FLAGRec,FLAGRB,FLAGDE

INTEGER $* 2$ D0,M0,Y0,H0,MI0,S0,HU0

INTEGER*2 DAY1,MONTH1,YEAR1,HOUR1,MINUTE1,SECOND1,HUND1

INTEGER *2 DAY2,MONTH2,YEAR2,HOUR2,MINUTE2,SECOND2,HUND2 
INTEGER *2 DHOUR,DMINUTE,DSECOND,DHUND

INTEGER *2 NGAMA,FLAGB,FLAGG,NCAP,NCOL,ICOL

INTEGER*2 ICX,ICCX,NPIC,CANAL1,CANAL2

INTEGER*4 TOTCEBD,TOTCFE,TOTCB,TOTCG,TTCSBe,TOTBETA,TOTGAMA

INTEGER*4 TOTCC,CANALMED,CANALMEE,CANMXCol,CNLmEDET,CNLmEBDT

INTEGER*4 NHIST,GT,GP,TOTec,BETAi,GAMAi,eci,TOTGMi,TOTeci,NEVGi

INTEGER*4 TOTecK,TOTecL,EBDZERO,ECBDZERO,ECecZERO,TTECBDZE

INTEGER *4 TOTRAN,TOTCAP,RARAN

REAL*4 CX,CCX,cr1,dr1,NCNG,INEF,ATIV,EKeK,EKeL,EKec,EBItemp

REAL*4 EBIetemp,kA,SIGMAALA,EGDT,GCX,RAIZEGDT,RAIZLN,COELIN

REAL*4 BC,BCTEMP,EEA,EEAL,WK,WL,PKLL,PKLX,EDESB,EDESC,KLL,KLX,KXY

REAL*4 EBmin,XCOL1,XCOL2

CHARACTER *70 CONST,ESQ,EFIB,NFERMI,EFIG,EFIGT,RANGE,OMC4PIBG,OBETA

CHARACTER $* 70$ OGAMA,OCOINCID,ONBNGNC1,ONBNGNC2,ONBNGNC3,ONBNGNC4

CHARACTER *70 OSOMABec,OMAESG,OMAESC,NPICO,OCONF,OEXCELC,OEXCELG

CHARACTER*15 NUCLIDEO

DATA CONST/'C:IEsquemalCONSTANT.DAT'/

DATA ESQ/'C:IEsquemalESQUEMA.DAT'/

DATA EFIB/'C: $:$ EsquemalEFIBETA.DAT'/

DATA NFERMI/'C:IEsquema|FERMI.DAT'/

DATA EFIG/'C:IEsquemalEFIGAMA.DAT'/

DATA EFIGT/'C:IEsquemalEFIGAMAT.DAT'/

DATA RANGE/'C:IEsquema\RANGE.DAT'/

DATA NPICO/'C:IEsquemalNPICOS.DAT'/

DATA OMC4PIBG/'C:|EsquemalMCBG.OUT'/

DATA OBETA/'C: $:$ Esquema $\backslash$ BETA.OUT'/

DATA OGAMA/'C:IEsquemalGAMA.OUT'/

DATA OCOINCID/'C: IEsquemalCOINCID.OUT'/

DATA ONBNGNC1/'C:IEsquemalNBGC1.OUT'/

DATA ONBNGNC2/'C:|EsquemalNBGC2.OUT'/

DATA ONBNGNC3/'C:|'EsquemalNBGC3.OUT'/

DATA ONBNGNC4/'C:IEsquemalNBGC4.OUT'/

DATA OSOMABec/'C: IEsquemalSOMABec.OUT'/

DATA OMAESG/'C:IEsquemalMAESTROG.OUT'/

DATA OMAESC/'C: IEsquemalMAESTROC.OUT'/

DATA OEXCELC/'C: $:$ EsquemalEXCELC.OUT'/

DATA OEXCELG/'C: $:$ Esquema\EXCELG.OUT'/

DATA OCONF/'C:IEsquemalCONFERE.OUT'/

$$
\vdots
$$




\section{APÊNDICE K - Arquivo RANGE.DAT ${ }^{[59]}$}

Vetor de energia e alcance de elétrons no Collodion, a $1^{\text {a }}$ coluna corresponde as energias e a $2^{\mathrm{a}}$ coluna corresponde ao alcance em $\mathrm{mg} / \mathrm{cm}^{2}$.

$0.0100 \quad 0.2809$

0.01250 .4158

$0.0150 \quad 0.5733$

$0.0175 \quad 0.7524$

$0.0200 \quad 0.9524$

0.02501 .4120

0.03001 .9490

$0.0350 \quad 2.5570$

$0.0400 \quad 3.2340$

0.04503 .9760

0.05004 .7810

$0.0550 \quad 5.6470$

$0.0600 \quad 6.5700$

0.07008 .5800

$\begin{array}{lll}0.0800 & 10.800\end{array}$

$0.0900 \quad 13.210$

$0.1000 \quad 15.800$

0.125022 .980

0.150031 .060

$\begin{array}{lll}0.1750 & 39.920\end{array}$

$0.2000 \quad 49.440$

0.250070 .160

$0.3000 \quad 92.680$

$0.3500 \quad 116.600$

$0.4000 \quad 141.600$

$0.4500 \quad 167.500$

$0.5000 \quad 194.100$

$0.5500 \quad 221.300$

$0.6000 \quad 249.000$

$0.7000 \quad 305.600$

$0.8000 \quad 363.200$

$0.9000 \quad 421.600$

$1.0000 \quad 480.600$

$1.2500 \quad 629.300$

1.5000778 .700

1.7500928 .000

$2.0000 \quad 1077.000$

$2.5000 \quad 1372.000$

$3.0000 \quad 1665.000$

3.50001953 .000

$4.0000 \quad 2239.000$

$4.5000 \quad 2521.000$ 


\section{REFERÊNCIAS BIBLIOGRÁFICAS}

[1] BAERG, A. P., Measurement of radioactivity disintegration rate by the coincidence method. Metrologia, 2 (1), p: 23-32, 1966.

[2] BAERG, A. P., Absolute measurement of radioactivity. Metrologia, $\underline{3}$ (4), p: 105$108,1967$.

[3] BAERG, A. P., The efficiency extrapolation method in coincidence counting. Nuclear Instruments and Methods. 112, p: 143-150, 1973.

[4] CAMPION, P. J., The standardization of radioisotopes by the beta-gamma coincidence method using high efficiency detectors. Int. J. Appl. Radiat. Iso., $\underline{4}$, p: 232-248, 1959.

[5] HOUTHERMANS, H.; MIGUEL, M., 4 $\pi \beta-\gamma$ coincidence counting for the calibration of nuclides with complex decay schemes. Int. J. Appl. Radiat. Isot. $\underline{13}$ p:137-142, 1962.

[6] KAWADA, Y., Extended applications and improvement of the $4 \pi \beta-\gamma$ coincidence method in the standardization of radionuclides. Res. of ETL. Japan, ETL-730, 1972.

[7] MOURA, L. P., Método de Coincidência Generalizado para a Medida Absoluta da Atividade de Radionuclídeos - Aplicação da Determinação do Coeficiente de Conversão Interna da Transição de $279 \mathrm{keV}$ do ${ }^{203} \mathrm{Tl}$. Tese de Doutorado apresentada à Universidade de Campinas, 1969.

[8] Da SILVA, M. A. L.; POLEDNA, R.; IWAHARA, A.; DA SILVA, C. J.; DELGADO, J. U.; LOPES, R. T., Standardization and decay data determinations of ${ }^{125} \mathrm{I},{ }^{54} \mathrm{Mn}$ e ${ }^{203} \mathbf{H g}$. In: INTERNATIONAL CONFERENCE ON RADIONUCLIDE METROLOGY AND ITS APPLICATIONS, $15^{\text {th }}$, Oxford, England, September 5-9, 2005. ICRM 2005, Book of abstracts, National Physical Laboratory (NPL) and International Committee for Radionuclide Metrology (ICRM).

[9] SAHAGIA, M., Standardization of ${ }^{\mathbf{9 9 m}}$ Tc. In: INTERNATIONAL CONFERENCE ON RADIONUCLIDE METROLOGY AND ITS APPLICATIONS, 15 ${ }^{\text {th }}$, Oxford, England, September 5-9, 2005. ICRM 2005, Book of abstracts, National Physical Laboratory (NPL) and International Committee for Radionuclide Metrology (ICRM).

[10] ROtetA, M.; TORAÑO, E. G.; BARQUERO, L. R., Standardization of ${ }^{\mathbf{1 8}} \mathbf{F}$ by Coincidence and LSC methods. In: INTERNATIONAL CONFERENCE ON RADIONUCLIDE METROLOGY AND ITS APPLICATIONS, 15 ${ }^{\text {th }}$, Oxford, England, September 5-9, 2005. ICRM 2005, Book of abstracts, National Physical Laboratory (NPL) and International Committee for Radionuclide Metrology (ICRM). 
[11] POMMÉ, S.; Altzitzoglou T.; Van AMMEL, R.; SIBBENS, G., Standardization of ${ }^{125} \mathrm{I}$ using seven techniques for radioactivity measurement. Nuclear Instruments and Methods in Physics Research Section A, Volume 544, Issue 3, p. 584-592, 2005.

[12] van WYNGAARDT, W. M.; SIMPSON, B. R. S., Absolute activity measurement of the electron-capture based radionuclides ${ }^{139} \mathrm{Ce},{ }^{125} \mathrm{I},{ }^{192} \mathrm{Ir}$ and ${ }^{65} \mathrm{Zn}$ by liquid scintillation coincidence counting. In: INTERNATIONAL CONFERENCE ON RADIONUCLIDE METROLOGY AND ITS APPLICATIONS, 15 ${ }^{\text {th }}$, Oxford, England, September 5-9, 2005. ICRM 2005, Book of abstracts, National Physical Laboratory (NPL) and International Committee for Radionuclide Metrology (ICRM).

[13] CESSNA, J. T.; SCHULTZ, M. K.; ZIMMERMAN, B. E., Update on the NIST triple-to-double coincidence ratio Liquid-Scintillation Counter. In: INTERNATIONAL CONFERENCE ON RADIONUCLIDE METROLOGY AND ITS APPLICATIONS, $15^{\text {th }}$, Oxford, England, September 5-9, 2005. ICRM 2005, Book of abstracts, National Physical Laboratory (NPL) and International Committee for Radionuclide Metrology (ICRM).

[14] HWANG, H. Y.; SUNG, K. S.; LEE, K. B.; LEE J. M.; PARK T. S., Standardization of Radionuclide by $\beta(\mathrm{LS})-\gamma$ Coincidence Counting using Geometry-Efficiency Variation Method. Applied Radiation and Isotopes, 64, p:1119-1123, OctoberNovember 2006.

[15] ARENILLAS, P.; CASSETTE, P., Implementation of the TDCR liquid scintillation method at CNEA-LMR, Argentina. Applied Radiation and Isotopes, 64, p:1500-1504, October-November 2006.

[16] YAMADA, T.; NAKAMURA, Y.; KAWADA, Y; SATO, Y.; HINO, Y., Standardization of ${ }^{152} \mathrm{Eu}$ and ${ }^{154} \mathrm{Eu}$ by $4 \pi \beta-4 \pi \gamma$ coincidence method and $4 \pi(\beta+\gamma)$ integral counting. Applied Radiation and Isotopes, 64, p:1220-1224, OctoberNovember 2006.

[17] BACCARELli, A. M.; DIAS, M. S.; KOSKINAS, M. F., Coincidence System For Standardization Of Radionuclides Using A 4-Pi Plastic Scintillator Detector. Applied Radiation and Isotopes, Grã-Bretanha, v. 58, p. 239-244, 2003.

[18] HILÁRIO, K. A. F., Desenvolvimento de métodos de medida de atividade empregando sistemas de coincidência para radionuclídeos que desintegram pela dupla emissão $\beta^{-}-\beta^{+}$/ captura eletrônica - aplicação na padronização do ${ }^{192} \mathbf{I r}$, ${ }^{152}$ Eu e ${ }^{186}$ Re. Tese de Doutorado apresentada ao Instituto de Pesquisas Energéticas e Nucleares, 2002.

[19] DiAS, M. S.; TAKedA, M. N.; KOSKInAS, M. F., Application of Monte Carlo simulation to the prediction of extrapolation curves in the coincidence technique. Applied Radiation and Isotopes, 64, p:1186-1192, October-November 2006.

[20] KOSKINAS, M. F.; MOREIRA, D. S.; TAKEDA, M. N.; SENEDA, J. A.; DIAS, M. S., Primary Standardization of ${ }^{72}$ Ga. Applied Radiation and Isotopes, 64, p:12251228, October-November 2006. 
[21] KOSKINAS, M. F.; SILVA, E. A.; YAMAZAKI, I. M.; DIAS, M. S., Standardization of ${ }^{241} \mathrm{Am}$ solution. Applied Radiation and Isotopes, 64, p:12381241, October-November 2006.

[22] MAIDANA, N. L.; TAKEDA, M. N.; DIAS, M. S.; KOSKINAS, M. F.; VANIN, V. R., Absolute measurement of ${ }^{242 g}$ Am sources activities in the ${ }^{241} \mathrm{Am}(\mathrm{n}, \gamma)$ crosssection determination-Improvement by simulation. Nuclear Instruments and Methods in Physics Research A, Volume 553, p. 559-568, 2005.

[23] TAKEDA, M. N.; DIAS, M. S.; KOSKINAS, M. F., Application of Monte Carlo Simulation to Cs-134 Standardization by Means of 4pi-beta-gamma Coincidence System. IEEE Transaction on Nuclear Science, vol. 52, No. 5, October 2005.

[24] MOREIRA. D. S., Padronização dos Radionuclídeos Multi-Emissores Gama ${ }^{166 m}$ Ho e $^{72}$ Ga e Determinação de Suas Intensidades Gama por Decaimento. Tese de Doutorado apresentada ao Instituto de Pesquisas Energéticas e Nucleares, 2005.

[25] TAKEDA, M. N.; DIAS, M. S.; KOSKINAS, M. F., Monte Carlo Simulation of Activity Measurements by Means of 4-pi-beta-gamma coincidence system. Brazilian Journal of Physics, São Paulo, v. 34, n. 3, p. 852-854, 2004.

[26] FERREIRA, C. R. P. P.; KOSKINAS, M. F.; DIAS, M. S., Standardization of Ca-45 Radioactive Solution by Tracing Method. Brazilian Journal of Physics, São Paulo, v. 34, n. 3, p. 939-941, 2004.

[27] DIAS, M. S., Calibração de um sistema de câmara de ionização de poço $4 \pi-\gamma$ para medidas de atividade de radionuclídeos. Dissertação de Mestrado apresentado à Escola Politécnica de São Paulo, 1978.

[28] FONSECA, K. A., Medida Absoluta da Atividade e Determinação da Taxa de Emissão Gama por Decaimento do ${ }^{126} \mathrm{I}$. Dissertação de Mestrado apresentada ao Instituto de Pesquisas Energéticas e Nucleares, 1997.

[29] KOSKINAS, M. F., Desenvolvimento de um sistema de coincidência para a medida absoluta da atividade de radionuclídeos empregando detetores de barreira de superfície. Tese de Doutorado apresentada ao Instituto de Pesquisas Energéticas e Nucleares, 1988.

[30] MULLER, J.W. and RYTZ, A., Report on the international comparison of dilution and source preparation methods by means of ${ }^{\mathbf{6 0}} \mathbf{C o}$. BIPM, setembro 1967.

[31] RATEL, G., Activity measurement of a ${ }^{75}$ Se solution in the frame of an international comparison (june 1992). CCEMRI(II)/93-14, maio 1993.

[32] RATEL, G., International comparison of activity measurement of a solution of ${ }^{109}$ Cd. CCEMRI(II)/87-7, maio 1987.

[33] RATEL, G., International comparison of activity measurements of a solution of ${ }^{125}$ I. CCEMRI(II)/89-2, abril 1989. 
[34] RYTS, A., Report on the international comparison of activity Measurement of a solution of ${ }^{139}$ Ce. Rapport BIPM - 77/4, 1977.

[35] RYTZ, A., International comparison of activity measurements of a solution of ${ }^{133}$ Ba. Rapport BIPM - 85/11, nov. 1985.

[36] RATEL, G., Results of the international comparison of activity measurements of ${ }^{152} \mathbf{E u}$, Nuclear Instruments \& Methods in Physics Research (aceito para publicação) 2001.

[37] SAZONOVA, T. E.; SEPMAN, S. V.; KARMALITSYN, N. I.; ZANEVSKY, A. V.; SHILNIKOVA, T. I.; GABITOV, R. M., Standardization of ${ }^{169} \mathbf{Y b}$ and $\gamma$-ray emission probability data. Applied Radiation and Isotopes, 52, p: 499-504, 2000.

[38] RAZDOLESCU, A. C.; GRIGORESCU, E. L.; SAHAGIA, M.; LUCA, A.; IVAN, C., Standardization of ${ }^{169} \mathrm{Yb}$ by the $4 \pi \beta-\gamma$ method. Applied Radiation and Isotopes, 52, p: 505-507, 2000.

[39] SAHAGIA, M.; RAZDOLESCU, A. C.; GRIGORESCU, E. L.; LUCA, A.; IVAN, C., Precise measurement of the activity of ${ }^{186} \mathrm{Re},{ }^{188} \mathrm{Re}$ radiopharmacuticals. Applied Radiation and Isotopes, 56, p: 349-356, 2002.

[40] EVANS, R. D. The Atomic Nucleus, McGraw-Hill Book Company, p.548, 1955.

[41] CROUTHAMEL, C. E., Applied Gamma-Ray Spectrometry. Second edition. Pergamon Press, 1970.

[42] KNOLL, G. F. Radiation Detection and Measurement. Third edition. John Wiley \& Sons, Inc., 2000.

[43] NATIONAL COUNCIL ON RADIATION PROTECTION AND MEASUREMENTS. Handbook of radioactivity measurements procedures. Report n. 58, Nov. 1978.

[44] BÉ, M. M; COURSOL, N.; DUCHEMIN, B.; LAMÉ, J.; MORILlON, F. P.; BROWNE, E.; CHECHEV, V.; HELMER, R.; SCHÖNFELD, E., Table de Radionucléides, Centre d'Etudes de Saclay, France, Commissariat à l'Énergie Atomique, 1999.

[45] DEBERTIN, K. and HELMER, R.G. Gamma and X-ray Spectrometry with Semiconductor Detectors, North-Holland, 1998.

[46] EVANS, R. D.; DAVISSON, C.M. Gamma-Ray Absorption Coefficients. Review of Modern Physics, vol. 24:2, april 1952.

[47] International Commission on Radiation Units and Measurements. Particle Counting in Radioactivity Measurements. ICRU Publ., Bethesda, Maryland, USA. November, (ICRU-Report 52), 1994. 
[48] LAVRAS, W., KOSKINAS, M.F., DIAS, M.S. AND FONSECA, K.A., Primary Standardization of ${ }^{51} \mathrm{Cr}$ radioactive solution. V Regional IRPA Congress Congress on Radiation Protection and Safety, II Iberian and Latin American Congress of Radiological Protection Societies, Recife - PE - Brasil, 04 de maio de 2001.

[49] RENNER, C. and PUGLIESI, R. "Report on the results of IPEN, São Paulo, Brazil, to the international comparison of ${ }^{134} \mathrm{Cs}$ sponsored by the Bureau International dès Poids et Mèsures", 1978.

[50] FIRESTONE, R. B., SHIRLEY, V.S., Table of Isotopes. $8^{\text {th }}$ ed. New York, 1996.

[51] BROWNE, R. and SHIRLEY, V. Table of Radioactive Isotopes, 8th. Ed., John Wiley \& Sons, New York, 1986.

[52] RYTZ, A.. International comparison of activity measurements of a solution of ${ }^{133}$ Ba (March 1984), Rapport BIPM-85/11, 1985.

[53] SÓBOL, I.M. Método de Monte Carlo. Moscou, Mir, 1976.

[54] CARTER, L.L.; CASHWELL, E.D. Particle Transport Simulation with the Monte Carlo Method. U. S. Energy Research and Development Administration, U.S.A., 1975.

[55] FOSHIMA, M. Uma Aplicação do Método de Monte Carlo à Física de Nêutrons. São Paulo, 1970. Dissertação Mestrado - Escola Politécnica da Universidade de São Paulo.

[56] VIEIRA, W.J. Simulação do Espectro de Deposição de Energia de Raios Gama em Detectores de NaI utilizando o Método de Monte Carlo. São Paulo, 1982. Dissertação Mestrado - Instituto de Pesquisas Energéticas e Nucleares.

[57] CASHWell, E.D.; EVEReTt, C.J. A Pratical Manual on the Monte Carlo Method for random walk problems. New York, Pergamon, 1959.

[58] ORNL, Monte Carlo N-Particle Transport Code System, MCNP4C, RSICC Computer Code Collection, Oak Ridge National Laboratory. Report CCC-700, 2001.

[59] http://physics.nist.gov/cgi-bin/star/e table.pl, acesso em 13/08/2003.

[60] SIEGBAHN K., Alpha-beta and gamma-ray spectroscopy, Amsterdam - North Holland, 1965.

[61] PENELOPE 2005, A Code System for Monte-Carlo Simulation of Electron and Photon Transport. http://www.nea.fr/abs/html/nea-1525.html, acesso em $15 / 11 / 2005$.

[62] PELOWITZ, D. B., editor, MCNPX ${ }^{\mathrm{TM}}$ user's manual, LA-CP-05-0369, version 2.5.0., http://alrads.nre.ufl.edu/TEMP/MCNPX 2.5.0_Manual.pdf, April 2005. 
[63] MORITA K.; MIYAHARA H.; OGATA Y.; KATOH K., Emission probability measurement of $\gamma$-ray of ${ }^{105} \mathbf{R h}$. Nuclear Instruments and Methods in Physics Research, Volume 540, Issues 2-3, 21 March 2005, Pages 324-327. 